\title{
Non-typhoidal human salmonellosis in Rio Grande do Sul, Brazil: A combined source attribution study of microbial subtyping and outbreak data
}

Neto, Waldemir Santiago; Leotti, Vanessa Bielefeldt; Pires, Sara Monteiro; Hald, Tine; Corbellini, Luis Gustavo

Published in:

International Journal of Food Microbiology

Link to article, DOI:

10.1016/j.ijfoodmicro.2020.108992

Publication date:

2021

Document Version

Peer reviewed version

Link back to DTU Orbit

Citation (APA):

Neto, W. S., Leotti, V. B., Pires, S. M., Hald, T., \& Corbellini, L. G. (2021). Non-typhoidal human salmonellosis in Rio Grande do Sul, Brazil: A combined source attribution study of microbial subtyping and outbreak data. International Journal of Food Microbiology, 338, [108992]. https://doi.org/10.1016/j.ijfoodmicro.2020.108992

\section{General rights}

Copyright and moral rights for the publications made accessible in the public portal are retained by the authors and/or other copyright owners and it is a condition of accessing publications that users recognise and abide by the legal requirements associated with these rights.

- Users may download and print one copy of any publication from the public portal for the purpose of private study or research.

- You may not further distribute the material or use it for any profit-making activity or commercial gain

- You may freely distribute the URL identifying the publication in the public portal 
1 Non-typhoidal human salmonellosis in Rio Grande do Sul, Brazil: A combined source attribution study 2 of microbial subtyping and outbreak data

3

4
Waldemir Santiago Neto ${ }^{a *}$, Vanessa Bielefeldt Leotti ${ }^{b}$, Sara Monteiro Piresc, Tine Hald ${ }^{d}$, Luís Gustavo Corbellini $^{\mathrm{a}}$

a Laboratory of Veterinary Epidemiology, Department of Preventive Veterinary Medicine, Universidade Federal do Rio Grande do Sul, Porto Alegre, Brazil

${ }^{\mathrm{b}}$ Department of Statistics, Institute of Mathematics and Statistics, Universidade Federal do Rio Grande do Sul, Porto Alegre, Brazil

${ }^{\mathrm{c}}$ Research Group for Risk-Benefit, National Food Institute, Technical University of Denmark, Kemitorvet, 2800 Kgs. Lyngby, Denmark

${ }^{\mathrm{d}}$ Research Group for Genomic Epidemiology, National Food Institute, Technical University of Denmark, Kemitorvet, 2800 Kgs. Lyngby, Denmark

*Corresponding author: EPILAB, Departamento de Medicina Veterinária Preventiva, Faculdade de Veterinária, Universidade Federal do Rio Grande do Sul (UFRGS), Av. Bento Gonçalves 9090, CEP 91540-000, Porto Alegre, RS, Brazil. Tel: +55 513308 6123. E-mail: wal sanet@hotmail.com (W. S. Neto). 


\section{ABSTRACT}

22

Salmonella spp. remains the most significant foodborne pathogen in south Brazil, but its epidemiology tends to change over time. Using official and surrogate data, a microbial subtyping 25 model attributed different Salmonella serovars to laying hens, pigs, broilers, and turkeys from 2005 to 2015 in Rio Grande do Sul (RS). Additional to the subtyping model, three sub-analyses of outbreak data attributed Salmonella spp. in humans to animal and non-animal food. Laying hens/eggs was the most important source of human salmonellosis in RS, with almost 40\% (159 cases; 95\% credibility interval, 43-247) attribution proportion, followed by pigs reared in Santa Catarina, a neighbor state (34.5\%). The Salmonella serovars Enteritidis and Typhimurium were the most common serovars involved. Source-related parameters had wide credibility intervals but showed a higher risk of illness from contaminated eggs than from the other three animal33 food sources. Analysis of the outbreak data corroborated the findings and indicated signs of decreasing importance for eggs and increasing importance for pork consumption.

Keywords: foodborne zoonoses, outbreaks, risk assessment, Salmonella source attribution, surveillance.

40 


\section{INTRODUCTION}

Non-typhoidal Salmonella (NTS) is among the major causal agents of self-limiting gastroenteritis, a local infection that causes diarrhea (Phu Huong Lan et al., 2016) and that can lead to chronic arthritis and invasive infections sometimes. Worldwide, more than that from any other enteric pathogen, Salmonella infections from contaminated food alone led to the loss of 6.43 million disability-adjusted life years in 2010, a World Bank metric that adds both the years of life lost and the years lost due to disability (Kirk et al., 2015). In Brazil, NTS is considered the most common foodborne bacterial zoonosis (Finger et al., 2019), including the southern region (Tondo and Ritter, 2012).

The consumption of raw eggs in homemade mayonnaise has been related to outbreaks of the Enteritidis serovar in Brazil (Wagner et al., 2013). However, a decrease in the importance of the Enteritidis serovar has been observed more recently (Reis et al., 2018), reflecting the possible effect of preventive measures adopted in food production chains (Ministry of Agriculture, Livestock, and Supply [MAPA], 2003). Commonly, changes in NTS epidemiology take place through ecological niche occupation by other serovars (Fernandes et al., 2006; Rodríguez et al., 2017). Salmonella Typhimurium, related to sporadic invasive salmonellosis cases (Reis et al., 2018), is found in most animal reservoirs and appears as a candidate, as well as emerging Salmonella serovars such as Heidelberg, Infantis and Newport (Penha Filho et al., 2019; Reis et al., 2018), distributed in specifically few reservoirs (e.g. broilers) (Voss-Rech et al., 2014). that some animal-derived foods act as reservoirs for particular serovars (Jackson et al., 2013; Kim, 
63 2010; Nüesch-Inderbinen et al., 2015). Source attribution models have been used for attributing

64 sporadic human cases or outbreaks of salmonellosis to specific sources in the last 20 years (David 65 et al., 2013; Domingues et al., 2012; Glass et al., 2016; Guo et al., 2011; Hald et al., 2004; Miller

66 et al., 2017; Mullner et al., 2009; Pires et al., 2010; Van Pelt et al., 1999; Wahlström et al., 2011).

67 The occurrence of foodborne outbreaks or sporadic infections caused by Salmonella could be 68 influenced by the serovars' capabilities for surviving and thriving in different hosts, and by the 69 likelihood of several food vehicles to establish an appropriate environment for Salmonella 70 growth, forming specific serovar-source combinations. Inter-serovar and food vehicle 71 differences can be estimated from complementary surveillance data and source attribution 72 models to improve understanding of the dynamics of human salmonellosis.

These models can be classified according to the point of attribution, specifically at

74 exposure or consumption, reservoir, or processing. While microbial subtyping approaches

75 attribute cases at the reservoir, analyses of data from outbreak investigations attribute it at 76 consumption. All microbial subtyping models require a collection of temporally and spatially 77 related isolates from humans and various sources. Therefore, an integrated foodborne disease 78 surveillance program that provides a collection of isolates from major animal reservoirs of the 79 foodborne pathogen would enable the construction of such models, based on the distribution of 80 isolate subtypes across different sources and humans (Hald et al., 2004). The subtyping of pathogens heterogeneously distributed among sources allows the 82 application of the Bayesian classification model. All human cases with unique pathogen subtypes 83 are assumed to originate from only one source. If subtypes isolated from only one source may 
84 originate from other sources, from which they were not detected due to the sensitivity of

85 surveillance systems, the importance of these unidentified sources in the salmonellosis burden

86 will be underestimated. Most source attribution approaches require parameter estimation, e.g.,

87 estimates of serovar and source-related factors, and take into account uncertainty around

88

89

90

91

92

93

94

95

96

97 isolates originating from poultry, turkeys, eggs, and pigs were considered the main sources of 98

99

estimates with credibility intervals (Crl) (Hald et al., 2004).

In Brazil and most developing countries, there is a dearth of information based on probabilistic source attribution reports, precisely where the burden of foodborne disease (FBD) is estimated to be higher (Li et al., 2019; Odeyemi, 2016; Schlundt et al., 2004). In the present study, we identify the most likely source-serovar combination of salmonellosis from 2005 to 2015 in the state of Rio Grande do Sul (RS), Brazil by modeling sporadic cases with the inclusion of the origin of the animal food sources according to its region of production across Brazil. RS is the southernmost state of Brazil and has a territorial extension of $281,730.2 \mathrm{~km}^{2}$; it is divided into 497 municipalities, and has 11.2 million inhabitants (Pessoa, 2017). In the present analysis, human infection. Complementarily, trends through time, between regions in RS and the locations of preparation/consumption were obtained by modeling data of Salmonella outbreaks.

\section{MATERIAL AND METHODS}

\subsection{General description of the data from humans and sources}

From 2011 to 2015, sporadic cases captured by Hospital Epidemiological Surveillance Nuclei (NVEH) from Global Salm-Surv (GSS)-linked hospitals covering 8 out of 19 Regional Health Coordination (CRS) from the sub-regions of RS resulted in 247 isolates of Salmonella spp. From 
1052005 to 2015, 324 outbreaks had information at the serovar level and were from all over the 106 state. From these, 153 were matched with serovars found in the studied sources. There were three available origins of data for the sources: Official monitoring for poultry,

108 turkey, and pigs; narrative review of literature of the prevalence data for pigs from Santa Catarina 109 (SC); and outbreak data for laying hens. For both humans and sources, antigenic characterization 110 and serovar identification were carried out in the National Reference Laboratory for Cholera and 111 Enteric Diseases, Oswaldo Cruz Institute (FIOCRUZ, Rio de Janeiro, Brazil) with the rapid slide 112 agglutination test using the somatic and flagellar antisera produced by the laboratory. The 113 diagram in Figure 1 illustrates the construction of the final dataset for source attribution. This 114 study was approved by the Research Ethics Committee at the Federal University of Rio Grande 115 do Sul under the Certificate of Presentation for Ethical Appreciation number $116 \quad 85788618.1 .0000 .5347$ on 5 July 2018.

\subsection{Microbial subtyping}

For the microbial subtyping approach, data were obtained from FBD outbreak

120 surveillance from the RS State Health Surveillance Center (CEVS) from 2005 to 2015 and from the

121 medical records of GSS-linked hospitals from 2011 to 2015. GSS was an international resource for

122 training and capacity building in FBD surveillance developed by the World Health Organization

123 (WHO) for improving the accurate identification and comparison of foodborne salmonellosis

124 (WHO, 2005). In Brazil, clinical samples were obtained from a laboratory-based surveillance 
125 network coordinated by the Bacteriology Division from the Central Public Health Laboratory, 126 augmenting the sensitivity of Salmonella human surveillance in RS. Data on the trade and transit of animal products (dos Santos et al., 2012) were obtained

128 from the State Secretariat of Agriculture, Livestock, and Supply (Supplementary Material S1) and 129 the distribution of different serovars in poultry meat and pigs raised in RS were obtained from 130 federal monitoring programs from MAPA (Tables B and C, Supplementary Material S1), and from 131 previous studies in one of the states that markets animal products to RS (da Silva et al., 2012; 132 Kich et al., 2011; Pissetti et al., 2012). Systematic surveillance of Salmonella spp. reported in animal reservoirs (e.g., broilers and 134 turkeys) can capture a wider range of serovars in asymptomatic hosts than in clinical 135 salmonellosis. To ensure flock health and the safety of poultry products in Brazil, MAPA has 136 implemented the Poultry Health National Plan, which includes Salmonella control initiatives in 137 the country's poultry industry. From 2004 to 2011, MAPA has implemented regular flock 138 surveillance for detecting the Salmonella serovars Enteritidis, Typhimurium, and others in 139 broilers before slaughter (Brasil, 2016). An exploratory study identified 16 serovars in pigs raised in RS, with only six in common 141 with human cases (Table C, Supplementary Material S1). In SC, a neighbor state of RS and a 142 prolific producer of pork, data on the occurrence of NTS were obtained from previous studies 143 from the Department of Preventive Veterinary Medicine of the Federal University of Rio Grande 144 do Sul (Table D, Supplementary Material S1). For beef cattle, the overall prevalence of Salmonella 145 spp. was $1.7 \%$ in 2015 and 2016 (personal communication, Karine Bordignon, MAPA). Although 146 beef seems to be a less important source of Salmonella in RS (da Silva et al. 2014), it is not 
147 negligible, as it accounts for invasive infections caused by serovars such as Dublin or prevalent 148 monophasic variant of Typhimurium; furthermore, traditionally, it could be consumed 149 undercooked. Its impact on human salmonellosis cases was assessed by analyzing outbreak data, 150 as serotyping was not performed until after the conclusion of this work.

Official monitoring data for laying hens were also not available for inclusion in the model.

152 However, as hen eggs are recognized as important sources of human Salmonella infections, the 153 serovar distribution data for reported egg-associated outbreaks were used for imputation in the 154 model. Among all egg-related Salmonella outbreaks for which specimens could be subtyped and 155 notified to CEVS from 2005 to 2015, the proportion of each Salmonella serovar was estimated 156 and used as input in the model (Figure 2). These represented $77.7 \%$ of the reported subtyped 157 Salmonella outbreaks.

We applied the Hald model for Salmonella using surveillance and monitoring data from 159 RS from 2005 to 2015 and estimated the number of sporadic and domestic salmonellosis cases 160 attributable to different food-producing animals (Hald et al., 2004). A sporadic case was defined 161 as a human case that could not be associated with a recognized FBD outbreak. For outbreak162 related cases, one outbreak contributed to the model with one sporadic case (e.g., index cases), 163 whereas the remaining cases associated with an outbreak were added to the final model results 164 after the modeling step, and attributed to the food involved in the outbreak, if known (Hald et 165 al., 2004). Travel-related cases were not included in the model because the history of traveling 166 before the onset of disease was not available for reported cases and they were considered 167 domestic (de Knegt et al., 2015b). 
The model assigns cases to animal reservoirs based on the distribution of Salmonella subtypes among animal hosts (Hald et al., 2004). The principle consists of comparing the number 170 of human cases caused by different serovars of a pathogen with the distribution of the same

171 serovars in different animal source foods. The model is constructed with collections of time- and 172 space-related isolates from various food and human sources, and the amount of food available 173 to the population is considered (De Knegt et al., 2015a; Hald et al., 2004). The expected number 174 of human cases per source and serovar was estimated by the following equation:

$$
\lambda_{i j}=M_{j} p_{i j} q_{i} a_{j}
$$

176 and

$177 o_{i}$ Poisson $\left(\Sigma_{j} \lambda_{i j}\right)$, where $\lambda_{i j}$ is the expected number of cases/year of serovar $i$ of source $j ; M_{j}$ is 178 the amount of source available for consumption (in 1000 tons); $p_{i j}$ the prevalence of serovar $i$ in 179 source $j ; q_{i}$ is the factor related to serovar $i ; a_{j}$ is source-related factor $j$; and $o_{i}$ is the observed 180 number of human cases of Salmonella serovar $i$. The expected number of sporadic and domestic 181 cases infected by Salmonella serovar $i$ was estimated based on:

$$
\lambda_{i}=\Sigma_{j} \lambda_{i j}
$$

which corresponds to the observed cases affected with serovar $i$ minus the observed cases 184 affected by serovar $i$ and known to be outbreak related. One case was subtracted for each 185 outbreak so that one outbreak contributed one sporadic case (Hald et al., 2004). As defined by Hald et al. (2004), the type-dependent factor $\left(q_{i}\right)$ summarizes the 187 characteristics of the serotype (survivability, virulence, pathogenicity), which determine its 
188 capacity for causing infection, and the source-dependent factor $\left(a_{j}\right)$ summarizes the 189 characteristics of the source (physical properties, preparation methods, processing procedures), 190 which determine its capacity for acting as a vehicle for Salmonella (Hald et al., 2004). Non191 informative uniform distributions with a positive lower bound were used for the source- and 192 serovar-dependent parameters $\left(a_{j}\right.$ and $\left.q_{i}\right)$. To ensure that the upper bounds allowed the 193 inclusion of all possible values for the parameter, posterior distributions were evaluated visually. 194 If distributions appeared to be disrupted, as in cases of arbitrary cut-off at the upper values, 195 uniform distributions were expanded until they reached the required ranges (Hald et al., 2004). The model was built using Markov chain Monte Carlo (MCMC) iterations with R software 197 (version R 3.3.3 2017, R Development core team) and packages rjags and coda (Supplementary 198 Material S2). Three independent Markov chains with widely dispersed initial values were 199 performed for 120,000 iterations after a 60,000-iteration burn-in period with a thinning of 1. 200 Autocorrelation of the Markov chain was checked as a function of delay, observing the similarity 201 between observations as a function of the time lag between them. In case autocorrelation 202 reached values close to 0 , it was assumed to be absent. Convergence was monitored using the 203 method developed by Gelman and Rubin (1992), and was considered to have occurred when the 204 chains reached stability and the variance between different chains was not larger than the 205 variance within each chain individually. String length was determined by running enough 206 iterations to ensure that the Monte Carlo errors for each parameter were $<5 \%$ of the later 207 standard deviation. 
Outbreak surveillance data were organized so that each reported outbreak corresponded

210 to one observation in the final dataset amenable for analysis. Each observation contained

211 information regarding the year of occurrence, the corresponding CRS, the population at risk, the

212 number of sick people and fatalities associated with the outbreak, the location of the outbreak,

213 and the food involved. Both confirmed (laboratory or epidemiologically) and suspected

214 outbreaks were used; for incomplete fields, data were included as unknown (e.g., unknown

215 source). Analytical epidemiological evidence corresponds to statistically significant associations

216 between a food item and human cases in the FBD outbreak from CEVS outbreak investigations.

217 Laboratory evidence implies that the causative agent has been detected by appropriate

218 laboratory methods at the food source or in the production and preparation environment. Non-

219 foodborne outbreaks were excluded from the dataset (e.g., person-to-person). The locations of

220 outbreaks were classified as private/household or public/general depending on where the

221 exposure took place (e.g., restaurants, hospitals) or where the food was produced or marketed

222 (e.g., supermarket).

223

Here, we classified food items using an adapted commodity-based hierarchical scheme

224 (Painter et al., 2009). The foods were divided into specific food categories of terrestrial animals,

225 plants, and seafood. The criterion used for defining if the food was complex was to have the

226 ingredients comprise $>1$ food category. Otherwise, the food was considered simple. The list of

227 ingredients for complex foods reported was obtained by reviewing recipes online: the top three

228 recipes from a Google search were chosen; for conflicting recipes, ingredients listed in at least

229 two recipes were selected. Unknown source outbreaks corresponded to most of the data. In 
230 cases without detailed information, animal-source ingredients were grouped under non231 specified "meat".

232 The use of outbreak data for the attribution of human FBD has been described previously

233 (Adak et al., 2005; Greig and Ravel, 2009; Pires et al., 2010), and the applied method is based on

234 the principles described by Pires et al. (2012). Data on reported outbreaks of Salmonella spp.

235 from RS occurring in 2006-2015 were used to attribute outbreaks of human salmonellosis to

236 specific food sources. We categorized implicated food into simple foods (i.e., constituted by

237 ingredients belonging to one single food category) and complex foods (i.e., constituted by

238 ingredients belonging to multiple food categories). In addition to the overall source attribution

239 model, a set of sub-analyses were performed, estimating the importance of sources in: (1) two

240 locations categories (general versus domestic outbreaks), (2) seven regions (Midwest, Central

241 eastern, Capital region, Northeast, Northwest, Southeast, and South-west), and (3) three

242 aggregated periods between 2006 and 2015.

243 The method used was based on the number of outbreaks caused by each food and was

244 aimed at attributing human salmonellosis to food. The outcome was the proportion of outbreaks

245 attributed to different food sources. To avoid potential overestimation of the proportion of

246 disease attributed to sources that caused large outbreaks, the number of ill people reported in

247 each outbreak was not used (Pires et al., 2010). Incriminated foods were classified based on the

248 number of ingredients, i.e., containing a single food category or multiple, complex foods,

249 designated by the defined criteria. If any food category was not implicated in a simple-food

250 outbreak, the category was not considered a possible source of complex-food outbreaks. This

251 premise may result in underestimation of the importance of some foods. However, our estimates 
252 are data-driven, and this limitation may be overcome by including larger amounts of data or with 253 analyses including an extended study period (Pires et al., 2010).

The proportion of human salmonellosis that can be attributed to each food source was

255 estimated based on the number of simple-food outbreaks caused by each source in the 256 ingredients (food categories) constituting complex foods, and based on the likelihood that each 257 of these categories was the cause of complex-food outbreaks, as described in Pires et al. (2012).

258 Briefly, simple-food outbreak data were summarized, and the proportion of outbreaks caused by 259 each category was used to define the distribution representing the probability that an outbreak $260 i$ was caused by source $j(P j)$, calculated as follows:

$$
P_{j} \operatorname{Beta}\left(\operatorname{source}_{j}+1, \text { totalS }-\operatorname{source}_{j}+1\right)
$$

262 where $\operatorname{source}_{j}$ is the total number of simple-food outbreaks caused by source $j$, and totalS is the 263 total number of simple-food outbreaks in the whole period and all regions. This probability was 264 estimated by source using information from all CRS and over the entire period. To calculate the 265 number of outbreaks attributed to each source, the simple-food outbreaks were assigned to the 266 respective simple-food categories. In turn, complex-food outbreaks were divided according to 267 each food category in the incriminated food in proportion to their likelihood of causing a simple268 food outbreak, calculated by $P$ j. Thus, complex-food outbreaks were attributed only to categories 269 that were incriminated in at least one simple-food outbreak. The model was built in WinBUGS 1.4 (http://www.mrc-bsu.cam.ac.uk/bugs/), which 271 defaults to the Gibbs sampler (MCMC simulation), and estimated $P_{j}$ (five independent Markov 272 chains with 60,000 iterations). For each chain, different initial values were used for $P j$, widely 273 dispersed in the target distribution (Supplementary Material S2). 


\section{3. RESULTS}

275

\subsection{General description of the data from humans and sources}

From 2011 to 2015, most sporadic cases captured from GSS in RS were that of serovars

277 Typhimurium, Enteritidis, and Infantis. Serovar Typhimurium surpassed serovar Enteritidis after 278 2011, and as did serovar Infantis from 2014 onwards (Reis et al., 2018). Additionally, from 2005

279 to 2015, 153 Salmonella outbreaks had information at serovar level state-wide, with the main 280 serovars implicated being Enteritidis and Typhimurium (Figure 2). While NVEH data were used 281 for the microbial subtyping model, outbreak data were used for both analyses, but with different 282 discrimination power. For the analysis of outbreak data, Salmonella was considered at species 283 level, resulting in a bigger sample for subset analyses.

Regarding data from putative Salmonella sources, 21 different serovars were obtained

285 from 128 samples from RS poultry and turkey farms, compared to 25 human serovars obtained 286 in NVEH (Reis et al., 2018). Five serovars were found in common in both surveillances. The main 287 serovars found in poultry meat species and pigs are shown in Tables B and C of Supplementary 288 Material S1, respectively.

\subsection{Microbial subtyping}

Serovars isolated from human cases were selected according to their occurrence in the

291 sources of production animals, which included 15 i serovars. Only one subspecies of Salmonella

292 enterica and three S. enterica enterica serovars were not detected in any of the sources and were 293 excluded from the analysis (S. enterica arizonae, and the S. enterica enterica serovars Muenchen, 294 Ohio, and Oranienburg). Regarding the sources, we divided broilers, turkeys, and pigs according 
295 to the state origin (i.e., Goiás [GO], Paraná, RS, or SC), which yielded eight $j$ sources. The 296 remaining source accounted was laying hens/eggs, with data from outbreaks. Considering the underrepresentation of cases (e.g., few data points in the period), we were unable to estimate the actual number of cases but could assign subtyped cases to the main 299 sources and estimate their relative importance. The most important sources of human 300 salmonellosis in RS between 2005 and 2015 estimated by the model were laying hens (eggs) at $30139.4 \%$ (159 cases, $95 \% \mathrm{Crl} 43-247$ ), followed by $34.5 \%$ of cases attributed to pigs reared in SC 302 (139 cases, 95\% Crl 60-187). Turkeys reared in other states and broilers reared in RS were 303 estimated as minor sources of Salmonella, contributing about $10 \%$ of cases each (turkeys raised 304 in GO, PR, and SC: 42 cases, 95\% Crl 15-125; chickens raised in RS: 40 cases, 95\% Crl 1-139). The most important Salmonella serovars isolated in the study period were Enteritidis, 306 responsible for $43.4 \%$ of cases (175 cases, 95\% Crl 150-202) and Typhimurium, contributing $30739.9 \%$ of cases (161 cases, 95\% Crl 137-187). Table 1 shows the estimates of the combinations 308 of serovars and animal sources. Of all Enteritidis serovar infections, $84.6 \%$ were attributed to 309 laying hens (148 cases, 95\% Crl 48-189), while $77 \%$ of cases caused by the Typhimurium serovar 310 originated from pigs raised in SC (124 cases, 95\% Crl 45-156). Among the emerging serovars, 311 Infantis in pigs (from SC) and Newport in turkeys (from GO, PR, and SC) were notable. The 312 observed number $\left(o_{i}\right)$ and the expected number $\left(\lambda_{i}\right)$ of Salmonella serovar cases are plotted in 313 Figure 3 . The fitness of the model could be explained by the absence of serovars distributed in 314 unknown sources. 
Eggs had the highest value for source-dependent factors $\left(a_{j}\right)$, followed by turkey meat, while pork had the lowest value (Table 2). However, eggs had a wide $\mathrm{Crl}$ range. This parameter

317 could indicate the likelihood of infection by consuming a particular unit (for example, $1 \mathrm{~kg}$ ) from 318 one source compared to another source. Table 2 presents the estimated values for the serovar319 dependent factors $\left(q_{i}\right)$. The results suggest that the ability of the Infantis serovar to survive food 320 processing and/or cause disease is about $40 \%$ greater than that of the Enteritidis serovar $(q=$ $32157.7,95 \% \mathrm{Crl}:$ 17.7-97.2), and about 50\% greater than that of the Typhimurium serovar.

\section{3.3. Analysis of outbreak data}

323 Outbreak investigation data were analyzed to estimate the proportion of salmonellosis

324 (species-level) outbreaks attributable to specific sources in the population of the different RS 325 regions. Sub-analyses estimated the importance of sources by location (comparing general and 326 domestic outbreaks), region (seven regions of RS), and over time (from 2006 to 2015). Due to 327 differences in data consistency, the temporal sub-analysis used 287 outbreaks, while the other 328 two sub-analyses used 817 outbreaks.

Figure 4 shows the proportion of foodborne outbreaks of salmonellosis from 330 epidemiological research data, which are stratified by 3-4-year periods. We estimated that eggs 331 were the most important source, but they showed a decline of about $13 \%$ in the period studied 332 and a difference of about $10 \%$ with pork in the final 4 years ( $>36 \%$ in the first 3 years). Other food 333 sources showed smaller variations, with a $5 \%$ increase in the proportion of chicken meat and a $3345 \%$ decrease in that of vegetables. 
Differences between outbreak locations were not significant (not shown). Outside the

336 home, the proportions were higher for eggs and meat in general; in the household, the 337 proportions for beef and pork were higher. Minor differences were found in other foods reported

338 in outbreak investigations. In the sub-analysis between regions, a pattern was observed in the 339 proportions of foods causing salmonellosis outbreaks, with unknown causes, eggs, and 340 vegetables predominating, and with small differences in importance (ranking) in the proportion 341 of the four meat source categories (Figure 5).

The probability $P_{j}$ that an outbreak was caused by source $j$ was higher for chicken and

343 pork, followed by cattle, eggs, meat, and vegetables (Figure 6). This means that, for example, in 344 an outbreak caused by a complex food containing pork and vegetables, the probability that pork 345 was the cause would be $30.1 \%$ (95\% Crl 19.6-42.0), and that of vegetables would be $6.4 \%$ (95\% $346 \mathrm{Crl}$ 1.2-1.5).

347 4. DISCUSSION

Data on human NTS are scarce in South America because of the lack of an effective 349 tracking system and efficient data recording in the countries, although the Salmonella 350 underreporting rate may be lower compared to other foodborne pathogens (Wheeler et al., 351 1999). Most foodborne gastroenteritis cases do not require hospitalization; from those that do 352 require hospitalization, the causative agent cannot be isolated from implicated food or clinical 353 samples in most cases, and neither are reported to the authorities. Regional representativeness 354 is heterogeneous, and more populated urban centers contribute to most of the reports (as seen 355 in Figueiredo et al., 2013). Previous studies have reported on Salmonella foodborne outbreaks in 
356 RS. Although different serovars were isolated from several foods, most salmonellosis outbreaks

357 were caused by S. Enteritidis, especially by strain SE86 (de Oliveira et al., 2006; Oliveira et al., 358 2007). On the other hand, there is a possible emergence of host-adapted, versatile $S$. 359 Typhimurium as one of the most prevalent foodborne pathogens worldwide (Hendriksen et al., 360 2011).

Both approaches were consistent in estimating eggs as one of the most important sources

362 of human salmonellosis in RS. Attribution estimates using outbreak data showed similar patterns

363 of the most important sources between the regions in RS, with emphasis on unknown sources, 364 vegetables, eggs, and broiler meat. The impact of beef was lower compared to other sources, 365 though its $P_{j}$ value was the third higher. The absence of estimates for this source in the subtyping 366 model can result in the misplacing of some cases when their true source is not included, 367 particularly in the estimation of those caused by ubiquitous serovars such as Typhimurium (de 368 Knegt et al., 2014a). The differences between regions may reflect data availability and quality 369 (e.g., readiness to investigate outbreaks; due to technical constraints or geographical distance) 370 and even different risk perceptions among outbreak investigators (i.e., reporting and 371 investigation bias). Regional differences may be more evident when the source attribution study 372 takes place in larger geographical areas (e.g., across countries) (Pires et al., 2012). Here, the trends observed in the outbreak model depict the importance of potato salad 374 with homemade mayonnaise in Salmonella cases, as described in previous studies (Costalunga 375 and Tondo, 2002; Wagner et al., 2013). The main factor contributing to the occurrence of 376 outbreaks was the consumption of products without sanitary inspection (i.e., clandestine, 377 informal production), mainly eggs, followed by food maintenance at room temperature for $>2$ 
378 hours (Costalunga and Tondo, 2002; Wagner et al., 2013). A cultural habit of the RS population is

379 to prepare homemade mayonnaise using raw eggs mixed with oil and warm cooked potato.

380 Often, the eggs are from small farms without sanitary inspection, increasing the probability of

381 Salmonella contamination. During the preparation, Salmonella can multiply in the eggs due to

382 the warm temperature and maintenance at room temperature. We attribute a significant

383 proportion of salmonellosis to vegetable consumption, which had lower $P_{j}$ weight, as a cause of

384 simple-food outbreaks. The results suggest that between $17 \%$ and $23 \%$ of salmonellosis cases are

385 attributed to this source, as seen in estimates from a worldwide data analysis of outbreak

386 investigations (Greig and Ravel, 2009). Vegetables accompanied the decreased importance of

387 eggs but could have been overestimated, possibly because potatoes are an ingredient in 388 homemade mayonnaise.

Despite the fewer data points in the analysis of trends through time, the relative contribution of eggs showed a declining pattern, although they were the most important source

391 in all periods, contrasting with the most significant increase of pork as a source of human 392 salmonellosis. The compulsory use of pasteurized eggs and municipality border surveillance could 393 be starting to control the incidence of egg-borne salmonellosis (ANVISA, 2009). This result raises 394 the hypothesis of the diminished importance of classical sources of Salmonella (e.g., eggs) in FBD 395 outbreaks. The decreasing importance of Gallus gallus has been observed in European countries 396 since the implementation of new control measures in chicken farms (e.g., the introduction of live 397 vaccines), improved hygiene, and education of consumers and food handlers (Hendriksen et al., 398 2011), especially after the implementation of national Salmonella control programs in chickens 399 and laying hens according to European Union Regulation No. 2016/2003. In the United States, an 
400

401

402

403

404

405

406

407

408

409

410

411

412

413

414

415

416

417

418

419

420

421

examination of trends in foodborne outbreaks by S. Enteritidis from 1979 to 2009 suggested that interventions initiated in the 1990s for curbing S. Enteritidis growth in eggs were essential for preventing further outbreaks (Wright et al., 2016).

Recently, pork consumption in Brazil has increased (ABPA, 2019). The analysis of outbreak data attributed an increasing proportion of cases to pork from the beginning of 2000 onwards, suggesting a change in NTS epidemiology. In RS, most pork production is related to sausage production rather than pork, which comes mainly from SC (personal communication, Carina dos Santos, Secretariat of Agriculture, Livestock and Rural Development of RS). Even with a similar prevalence of Salmonella in pigs from RS and SC (Tables C and D, Supplementary Material S1), the type of product and the higher amount of pork consumed from SC (Table A, Supplementary Material S1) may explain the observed results, raising the hypothesis of a potential regional chain from SC. Conversely, the incorporation of parameter $M_{j}$ did not affect the attribution estimates of previous applications of the modified Hald model (Mullner et al., 2009; Mughini-Gras et al., 2014a).

There is growing evidence that pigs play a major role as sources of human salmonellosis and that the most likely route of transmission is poor handling and consumption of contaminated pigs (Mughini-Gras et al., 2014b). Just as poultry are the probable reservoirs of S. Enteritidis, pigs are the most likely reservoir of S. Typhimurium (De Knegt et al., 2015), in addition to its singlephase variant 4,[5],12:i:- and S. Derby (Hoelzer et al., 2011). S. Typhimurium seems to be more related to cases requiring hospitalization (Reis et al., 2018) and should be monitored, including its single-phase variant and antimicrobial resistance levels, as well as other emerging Salmonella serovars such as Infantis, Heidelberg, and Newport (Penha Filho et al., 2019; Reis et al., 2018). 
It is assumed that the estimated values of the source and pathogen-related factors

423 consider both the consistency of the surveillance systems and the intrinsic ability of serovars to

424 cause disease in humans. However, it should be interpreted relatively, and not as an absolute

425 measure. Due to the relatively few data points available for the period of analysis, the Crl were

426 widespread, making the ranking less interpretable. The inclusion of data from different

427 surveillance systems (e.g., CEVS and NVEH) and periods could reflect changes in the capacity for

428 detecting different kinds of pathogenicity (e.g., generally mild, widespread foodborne outbreaks

429 or hospitalization-related, sporadic cases), consumption/exposure habits, and the (effectiveness

430 of) implementation of animal health programs, on Salmonella cases. It is possible that the fact

431 that pork is typically consumed well-cooked influences the lower values for its source-dependent

432 parameter. Otherwise, the higher value for eggs and turkeys agrees with a previous study 433 covering European countries (de Knegt et al., 2015a).

Limitations

We compared the results from the combination of a microbiological and an

epidemiological source attribution method to overcome their inherent limitations. The estimates

437 account for differences in periods and populations at risk, the point of attribution, the sources

438 investigated, and the serovar/species discrimination power. The applicability of periodic

439 surveillance data must include the consideration that production prevalence estimates are

440 imperfect substitutes for actual exposure and that unrepresented sources contribute to the

441 impact of disease on the human population (Ranta et al., 2011). As time series collections

442 increase, time-prevalence models improve and provide estimates even for periods with missing

443 data. When insufficient surveillance or monitoring data of the herd/flock are available for a 
444 source, slaughter samples should be surveyed and their quality as substitutes assessed (de Knegt 445 et al., 2015b). For most sources, the data were from official monitoring programs, which do not 446 consider informal, uninspected food production.

Most salmonellosis outbreaks originate from eggs, and consumption of animal-source

448 food without inspection is a risk factor of foodborne outbreaks in RS (Wagner et al., 2013). In the 449 absence of official data for laying hens, we assumed that the proportion of egg-related 450 Salmonella outbreaks could partially represent exposure to this source. If the hypothesis of lower 451 Salmonella prevalence in commercial, inspected laying hens holds, then the input of egg452 associated Salmonella outbreaks could lead to overestimation of salmonellosis cases attributed 453 to laying hens. This compromises interpretations to some degree, and they could be improved 454 by including the data origins of more sources (e.g., with inspection).

The lower discriminatory subtyping data could result in less accurate estimates when 456 attributing salmonellosis cases (Pires and Hald, 2010), particularly when it comes to ubiquitous 457 types such as S. Typhimurium and S. Enteritidis (De Knegt et al., 2015a). Highly discriminatory 458 methods are a suitable solution for (single) source attribution for a cluster of human cases, as 459 would be the case in timely outbreak investigations. To relate groups of bacterial strains with 460 particular reservoirs/sources and then attribute sporadic human cases to these sources, models 461 based on both serovars and phage types allow a process to identify sufficient diversity between 462 strains from humans and causative sources to a degree where epidemiological relatedness can 463 be assumed (de Knegt et al., 2016). 
Furthermore, there is a need to consider additional sources and transmission routes when

465 choosing a source attribution approach, depending on the public health issues to be addressed, 466 such as travel-related cases and direct contact contamination. Improved investigations of

467 sporadic and outbreak-related cases, e.g., regarding travel history, which may have significant 468 relative importance, as is the case in countries with national salmonellosis control (Hald et al., 469 2004; de Jong and Ekdahl 2006), and comparative exposure assessment could add value to 470 estimates. Conversely, a systematic review of case-control studies may underestimate the 471 importance of travel and assign cases to other exposures included in the questionnaires, such as 472 direct contact with domestic animals and environmental sources (Pires, 2009). The inclusion of 473 additional relevant issues could provide a comprehensive assessment of multidimensional food474 related hazards, such as interactions between microbial ecology, antibiotics use, and the 475 effectiveness of other disease control programs.

476 Conclusions

The combination of source attribution approaches has proven to be a valuable tool for 478 quantifying the importance of different food sources in human salmonellosis in RS, even with the 479 use of sparse data. Although the data were limited, which resulted in uncertainties, we believe 480 that our study is a crucial first step towards investigating the relative contribution of different 481 sources of salmonellosis in the state. It is also noteworthy to highlight data gaps and research 482 needs, which will pave the way for improving source attribution efforts. Analysis of improved 483 integrated surveillance data could yield more accurate results, cover more sources and larger 484 areas, and thus better assist risk mitigation decisions, guide priority interventions from source to 
485 consumption, and measure the effects of current control programs on the burden of Salmonella 486 on humans.

487

488

489 Conflict of interest statement

490 There authors report no conflicts of interest.

491

492

493 Acknowledgments

494 Financial support was provided by CNPq/Brazil through the Doctoral Training Program (process

495 \# 163718/2015-6). We are especially grateful to Denise Maria Silva Figueiredo, Loeci Timm, and

496 all official veterinarians from MAPA (Ministério da Agricultura, Pecuária, e Abastecimento)

497 enrolled in the microbiological baseline studies. 


\section{References}

ABPA, 2019. Relatório Anual 2019 [WWW Document]. Relatório Anu. 2019. http://abpabr.org/relatorios/ (accessed 08.08.2019).

Adak, G.K., Meakins, S.M., Yip, H., Lopman, B.A., O’Brien, S.J., 2005. Disease risks from foods, England and Wales, 1996-2000. Emerg. Infect. Dis. 11(3), 365-72. https://doi.org/10.3201/eid1103.040191

ANVISA, 2009. Resolução n 35, de 17 de junho de 2009.

Brasil, 2016. Ministério da Agricultura, Pecuária e Abastecimento. Instrução Normativa n²0, de 21 de outubro de 2016. Diário Of. da União.

Costalunga, S., Tondo, E.C., 2002. Salmonellosis in Rio Grande do Sul, Brazil, 1997 to 1999. Brazilian J. Microbiol. 33, 342-46. https://doi.org/10.1590/S1517-83822002000400013

da Silva, F.F.P., Horvath, M.B., Silveira, J.G., Pieta, L., Tondo, E.C., 2014. Occurrence of Salmonella spp. and generic Escherichia coli on beef carcasses sampled at a Brazilian slaughterhouse. Braz. J. Microb. 45(1), 17-23. https://doi.org/10.1590/S151783822014005000037

da Silva, L.E., Dias, V., Ferronatto, A., Guerra, P., Berno, L., Triches, N., Kich, J.D., Corbellini, L.G., Cardoso, M., 2012. Longitudinal dissemination of Salmonella enterica clonal groups through the slaughter process of Salmonella-positive pig batches. J. Food Prot. 75(9), 15808. https://doi.org/10.4315/0362-028x.jfp-11-515

David, J.M., Sanders, P., Bemrah, N., Granier, S.A., Denis, M., Weill, F.X., Guillemot, D., Watier, L., 2013. Attribution of the French human salmonellosis cases to the main food-sources according to the type of surveillance data. Prev. Vet. Med. 110(1), 12-27. https://doi.org/10.1016/j.prevetmed.2013.02.002

de Jong, B., Ekdahl, K., 2006. The comparative burden of salmonellosis in the European Union member states, associated and candidate countries. BMC Public Health. 6, 4. 10.1186/1471-2458-6-4

de Knegt, L. V., Pires, S.M., Hald, T., 2015a. Attributing foodborne salmonellosis in humans to animal reservoirs in the European Union using a multi-country stochastic model. Epidemiol. Infect. 143(6), 1175-86. https://doi.org/10.1017/S0950268814001903

de Knegt, L.V., Pires, S.M., Hald, T., 2015b. Using surveillance and monitoring data of different origins in a Salmonella source attribution model: a European Union example with challenges and proposed solutions. Epidemiol. Infect. 143, 1148-65. $10.1017 /$ S0950268814000429

de Knegt, L. V., Pires, S.M., Löfström, C., Sørensen, G., Pedersen, K., Torpdahl, M., Nielsen, E.M., Hald, T., 2016. Application of molecular typing results in source attribution models: the case of multiple locus variable number tandem repeat analysis (MLVA) of Salmonella isolates obtained from integrated surveillance in Denmark. Risk Anal. 36(3), 571-88. 

https://doi.org/10.1111/risa.12483

de Oliveira, F.A., Brandelli, A., Tondo, E.C., 2006. Antimicrobial resistance in Salmonella enteritidis from foods involved in human salmonellosis outbreaks in southern Brazil. New Microbiol. 29, 49-54. https://doi.org/10.1590/S1517-838220100004000015

Domingues, A.R., Pires, S.M., Halasa, T., Hald, T., 2012. Source attribution of human salmonellosis using a meta-analysis of case-control studies of sporadic infections. Epidemiol. Infect. 140(6), 959-69. https://doi.org/10.1017/S0950268811002172

dos Santos, D.V., Hein, H.E., Machado, G., 2012. Análise de ingresso de animais de produção e seus produtos no Estado do Rio Grande do Sul no ano de 2011. Porto Alegre. https://www.agricultura.rs.gov.br/upload/arquivos/201611/25103715-see-artigo14analise-do-ingresso-de-animais-de-producao-e-seus-produtos-no-estado-do-rio-grandedo-sul-no-ano-de-2011.pdf (accessed 08.08.2019).

Fernandes, S.A., Tavechio, A.T., Ghilardi, Â.C.R., Dias, Â.M.G., De Almeida, I.A.Z.C., De Melo, L.C.V., 2006. Salmonella serovars isolated from humans in São Paulo State, Brazil, 19962003. Rev. Inst. Med. Trop. Sao Paulo. 48(4), 179-84. https://doi.org/10.1590/S003646652006000400001

Figueiredo, D.M.S., Timm, L.N., Cecconi, M.C.P., Both, J.M.C., Soeiro, M.L.T., Ramos, R.C., Haas, S., Longaray, S.M., 2013. Programa de Vigilância Epidemiológica das Doenças de Transmissão Hídricas e Alimentares - VE-DTHA, Boletim Epidemiológico. https://www.cevs.rs.gov.br/upload/arquivos/201903/11165255-n-3-setembro.pdf (accessed 06.06.2019).

Finger, J.A.F.F., Baroni, W.S.G.V., Maffei, D.F., Bastos, D.H.M., Pinto, U.M., 2019. Overview of foodborne disease outbreaks in Brazil from 2000 to 2018. Foods. 8(10), 434. https://doi.org/10.3390/foods8100434

Gelman, A., Rubin, D.B., 1992. Inference from iterative simulation using multiple sequences. Stat. Sci. 7(4), 457-72. https://doi.org/10.1214/ss/1177011136

Glass, K., Fearnley, E., Hocking, H., Raupach, J., Veitch, M., Ford, L., Kirk, M.D., 2016. Bayesian source attribution of salmonellosis in south Australia. Risk Anal. 36(3), 561-70. https://doi.org/10.1111/risa.12444

Greig, J.D., Ravel, A., 2009. Analysis of foodborne outbreak data reported internationally for source attribution. Int. J. Food Microbiol. 130(2), 77-87. https://doi.org/10.1016/j.ijfoodmicro.2008.12.031

Guo, C., Hoekstra, R.M., Schroeder, C.M., Pires, S.M., Ong, K.L., Hartnett, E., Naugle, A., Harman, J., Bennett, P., Cieslak, P., Scallan, E., Rose, B., Holt, K.G., Kissler, B., Mbandi, E., Roodsari, R., Angulo, F.J., Cole, D., 2011. Application of Bayesian techniques to model the burden of human salmonellosis attributable to U.S. food commodities at the point of processing: adaptation of a Danish model. Foodborne Pathog. Dis. 8(4), 509-16. https://doi.org/10.1089/fpd.2010.0714 
Hald, T., Vose, D., Wegener, H.C., Koupeev, T., 2004. A Bayesian approach to quantify the contribution of animal-food sources to human salmonellosis. Risk Anal. 24(1), 255-69. https://doi.org/10.1111/j.0272-4332.2004.00427.x

Hendriksen, R.S., Jensen, A.B., Karlsmose, S., Lo Fo Wong, D.M.A., Wegener, H.C., Aarestrup, F.M., Vieira, A.R., 2011. Global monitoring of Salmonella serovar distribution from the World Health Organization Global Foodborne Infections Network Country Data Bank: results of quality assured laboratories from 2001 to 2007. Foodborne Pathog. Dis. 8(8), 887-900. https://doi.org/10.1089/fpd.2010.0787

Hoelzer, K., Switt, A.I.M., Wiedmann, M., 2011. Animal contact as a source of human nontyphoidal salmonellosis. Vet. Res. 42(1), 34. https://doi.org/10.1186/1297-9716-42-34

Jackson, B.R., Griffin, P.M., Cole, D., Walsh, K.A., Chai, S.J., 2013. Outbreak-associated Salmonella enterica serotypes and food commodities, United States, 1998-2008. Emerg. Infect. Dis. 19(8), 1239-44. https://doi.org/10.3201/eid1908.121511

Kich, J.D., Coldebella, A., Morés, N., Nogueira, M.G., Cardoso, M., Fratamico, P.M., Call, J.E., Fedorka-Cray, P., Luchansky, J.B., 2011. Prevalence, distribution, and molecular characterization of Salmonella recovered from swine finishing herds and a slaughter facility in Santa Catarina, Brazil. Int. J. Food Microbiol. 151(3), 307-13. https://doi.org/10.1016/j.ijfoodmicro.2011.09.024

Kim, S., 2010. Salmonella serovars from foodborne and waterborne diseases in Korea, 19982007: total isolates decreasing versus rare serovars emerging. J. Korean Med. Sci. 25(12), 1693-9. https://doi.org/10.3346/jkms.2010.25.12.1693

Kirk, M.D., Pires, S.M., Black, R.E., Caipo, M., Crump, J.A., Devleesschauwer, B., Döpfer, D., Fazil, A., Fischer-Walker, C.L., Hald, T., Hall, A.J., Keddy, K.H., Lake, R.J., Lanata, C.F., Torgerson, P.R., Havelaar, A.H., Angulo, F.J., 2015. World Health Organization estimates of the global and regional disease burden of 22 foodborne bacterial, protozoal, and viral diseases, 2010: a data synthesis. PLoS Med. 12(12), e1001940. https://doi.org/10.1371/journal.pmed.1001921

Li, M., Havelaar, A.H., Hoffmann, S., Hald, T., Kirk, M.D., Torgerson, P.R., Devleesschauwer, B., 2019. Global disease burden of pathogens in animal source foods, 2010. PLoS One. 14(6), e0216545. https://doi.org/10.1371/journal.pone.0216545

MAPA, 2003. Instrução Normativa $N^{\circ} 78$, de 3 de novembro de 2003. Diário Of. da União.

Miller, P., Marshall, J., French, N., Jewell, C., 2017. sourceR: Classification and source attribution of infectious agents among heterogeneous populations. PLoS Comput. Biol. 30(13), e1005564. https://doi.org/10.1371/journal.pcbi.1005564

Mughini-Gras, L., Barrucci, F., Smid, J.H., Graziani, C., Luzzi, I., Ricci, A., Barco, L., Rosmini, R., Havelaar, A.H., Van Pelt, W., Busani, L., 2014a. Attribution of human Salmonella infections to animal and food sources in Italy (2002-2010): adaptations of the Dutch and modified Hald source attribution models. Epidemiol. Infect. 142(5), 1070-82. 
Mughini-Gras, Lapo, Enserink, R., Friesema, I., Heck, M., Van Duynhoven, Y., Van Pelt, W., 2014b. Risk factors for human salmonellosis originating from pigs, cattle, broiler chickens and egg laying hens: A combined case-control and source attribution analysis. PLoS One. 9(2), e87933. https://doi.org/10.1371/journal.pone.0087933

Mullner, P., Jones, G., Noble, A., Spencer, S.E.F., Hathaway, S., French, N.P., 2009. Source attribution of food-borne zoonoses in New Zealand: a modified Hald model. Risk Anal. 29(7), 970-84. https://doi.org/10.1111/j.1539-6924.2009.01224.x

Nüesch-Inderbinen, M., Cernela, N., Althaus, D., Hächler, H., Stephan, R., 2015. Salmonella enterica serovar Szentes, a rare serotype causing a 9-month outbreak in 2013 and 2014 in Switzerland. Foodborne Pathog. Dis. 12(11), 887-90. https://doi.org/10.1089/fpd.2015.1996

Odeyemi, O.A., 2016. Public health implications of microbial food safety and foodborne diseases in developing countries. Food Nutr. Res. 60, 29819. https://doi.org/10.3402/fnr.v60.29819

Oliveira, F.A. de, Frazzon, A.P.G., Brandelli, A., Tondo, E.C., 2007. Use of PCR-ribotyping, RAPD, and antimicrobial resistance for typing of Salmonella Enteritidis involved in food-borne outbreaks in Southern Brazil. J. Infect. Dev. Ctries. 1(2), 170-6.

Painter, J.A., Ayers, T., Woodruff, R., Blanton, E., Perez, N., Hoekstra, R.M., Griffin, P.M., Braden, C., 2009. Recipes for foodborne outbreaks: a scheme for categorizing and grouping implicated foods. Foodborne Pathog. Dis. 6(10), 1259-64. https://doi.org/10.1089/fpd.2009.0350

Penha Filho, R.A.C., Ferreira, J.C., Kanashiro, A.M.I., Berchieri Junior, A., Darini, A.L. da C., 2019. Emergent multidrug-resistant nontyphoidal Salmonella serovars isolated from poultry in Brazil coharboring blaCTX-M-2 and qnrB or blaCMY-2 in large plasmids. Diagn. Microbiol. Infect. Dis. 95(1), 93-8. https://doi.org/10.1016/j.diagmicrobio.2019.04.003

Pessoa, M. L. (Org.). População do RS. In: Atlas FEE. Porto Alegre: FEE, 2017. http://atlas.fee.tche.br/rio-grande-do-sul/demografia/populacao-do-rs/ (accessed 23.06.2020).

Phu Huong Lan, N., Le Thi Phuong, T., Nguyen Huu, H., Thuy, L., Mather, A.E., Park, S.E., Marks, F., Thwaites, G.E., Van Vinh Chau, N., Thompson, C.N., Baker, S., 2016. Invasive nontyphoidal Salmonella infections in Asia: clinical observations, disease outcome and dominant serovars from an Infectious Disease Hospital in Vietnam. PLoS Negl. Trop. Dis. 10(8), e0004857. https://doi.org/10.1371/journal.pntd.0004857

Pires, S.M., 2009. Attributing human salmonellosis and campylobacteriosis to food, animal and environmental sources. Fac. Life Sci.

Pires, S.M., Hald, T., 2010. Assessing the differences in public health impact of Salmonella subtypes using a Bayesian microbial subtyping approach for source attribution. Foodborne 
Pathog. Dis. 6(4), 417-24. https://doi.org/10.1089/fpd.2009.0369

Pires, S.M., Vieira, A.R., Perez, E., Wong, D.L.F., Hald, T., 2012. Attributing human foodborne illness to food sources and water in Latin America and the Caribbean using data from outbreak investigations. Int. J. Food Microbiol. 152(3), 129-38. https://doi.org/10.1016/j.ijfoodmicro.2011.04.018

Pires, S.M., Vigre, H., Makela, P., Hald, T., 2010. Using outbreak data for source attribution of human salmonellosis and campylobacteriosis in Europe. Foodborne Pathog. Dis. 7(11), 1351-61. https://doi.org/10.1089/fpd.2010.0564

Pissetti, C., Werlang, G.O., Biesus, L.L., Kich, J.D., Cardoso, M.R. de I., 2012. Salmonella enterica and Listeria monocytogenes detection on pre-chill pig carcasses. Acta Sci. Vet. 40(4), 1-8. http://www.ufrgs.br/actavet/40-4/PUB\%201071.pdf

Ranta, J., Matjushin, D., Virtanen, T., Kuusi, M., Viljugrein, H., Hofshagen, M., Hakkinen, M., 2011. Bayesian temporal source attribution of foodborne zoonoses: Campylobacter in Finland and Norway. Risk Anal. 31(7), 1156-71. https://doi.org/10.1111/j.15396924.2010.01558.x

Reis, R.O. dos, Souza, M.N., Cecconi, M.C.P., Timm, L., Ikuta, N., Simon, D., Wolf, J.M., Lunge, V.R., 2018. Increasing prevalence and dissemination of invasive nontyphoidal Salmonella serotype Typhimurium with multidrug resistance in hospitalized patients from southern Brazil. Brazilian J. Infect. Dis. 22(5), 424-32. https://doi.org/10.1016/j.bjid.2018.08.002

Rodríguez, E.C., Díaz-Guevara, P., Moreno, J., Bautista, A., Montaño, L., Realpe, M.E., della Gaspera, A., Wiesner, M., 2017. Laboratory surveillance of Salmonella enterica from human clinical cases in Colombia 2005-2011. Enferm. Infecc. Microbiol. Clin. 35(7), 17-425. https://doi.org/10.1016/j.eimc.2017.02.023

Schlundt, J., Toyofuku, H., Jansen, J., Herbst, S.A., 2004. Emerging food-borne zoonoses. OIE Rev. Sci. Tech. 23(2), 513-33. https://doi.org/10.20506/rst.23.2.1506

Tondo, E.C., Ritter, A.C., 2012. Salmonella and salmonellosis in Southern Brazil: A review of the last decade. In: Salmonella: Classification, Genetics and Disease Outbreaks. Nova Science Publishers.

Van Pelt, W., van de Giessen, A.W., Van Leeuwen, W.J., Al, E., 1999. Oorsprong, omvang en kosten van humane salmonellose. Deel 1. Oorsprong van human salmonellose met betrekking tot varken, rund, kip, ei en overige bronnen. Infectiezikten Bulletin 1999; 10.

Voss-Rech, D., Vaz, C.S., Alves, L., Coldebella, A., Leão, J.A., Rodrigues, D.P., Back, A., 2015. A temporal study of Salmonella enterica serotypes from broiler farms in Brazil. Poult Sci. 94(3), 433-41. https://doi: 10.3382/ps/peu081.

Wagner, V.R., Silveira, J.B., Tondo, E.C., 2013. Salmonelloses in the state of Rio Grande do Sul, southern Brazil, 2002 to 2004. Brazilian J. Microbiol. 44(3), 723-9. https://doi.org/10.1590/S1517-83822013005000064 
687 Wahlström, H., Andersson, Y., Plym-Forshell, L., Pires, S.M., 2011. Source attribution of human https://doi.org/10.1017/S0950268810002293

Wheeler, J.G., Sethi, D., Cowden, J.M., Wall, P.G., Rodrigues, L.C., Tompkins, D.S., Hudson, M.J., Roderick, P.J., 1999. Study of infectious intestinal disease in England: rates in the community, presenting to general practice, and reported to national surveillance. The Infectious Intestinal Disease Study Executive. BMJ. 318(7190), 1046-50. https://doi.org/10.1136/bmj.318.7190.1046

WHO, 2005. WHO Global Salm-Surv A surveillance network for foodborne diseases. International Food Safety Authorities Network (INFOSAN). https://www.who.int/foodsafety/fs management/No 06 GSS Oct05 en.pdf (accessed 05.04.2020).

Wright, A.P., Richardson, L., Mahon, B.E., Rothenberg, R., Cole, D.J., 2016. The rise and decline in Salmonella enterica serovar Enteritidis outbreaks attributed to egg-containing foods in the United States, 1973-2009. Epidemiol. Infect. 144(4), 810-9. https://doi.org/10.1017/S0950268815001867 
704

705

706

\section{Tables}

Table 1. Estimates of expected numbers of cases for major combinations of serovars and sources of livestock from different states causing human salmonellosis in Rio Grande do Sul from 2005 to 2015.

\begin{tabular}{lcc}
\hline Serovar/Source (Origin State ${ }^{\mathrm{a}}$ ) & Median & $95 \% \mathrm{Cr}^{\mathrm{b}}$ \\
\hline Enteritidis/Eggs (RS) & 148 & $(48-189)$ \\
Typhimurium/Pigs (SC) & 124 & $(45-156)$ \\
Enteritidis/Broilers (RS) & 17 & $(1-100)$ \\
Infantis/Pigs (SC) & 16 & $(3-32)$ \\
Typhimurium/Turkeys (GO/PR/SC) & 12 & $(3-85)$ \\
Newport/Turkeys (GO/PR/SC) & 11 & $(5-18)$ \\
Typhimurium/Pigs (RS) & 10 & $(3-12)$ \\
Typhimurium/Eggs (RS) & 8 & $(2-40)$
\end{tabular}

${ }^{a}$ GO: Goiás; PR: Paraná; RS: Rio Grande do Sul; SC: Santa Catarina

${ }^{b}$ Credibility interval

Table 2. Estimated values for source- and serovar-dependent factors: $a_{j}{ }^{\mathrm{a}}$ and $q_{i}$, which summarize, respectively, the capacity of the source to act as a vehicle for Salmonella, and the likelihood of a Salmonella serovar to cause infection. The ranking is based on the point estimate.

\begin{tabular}{lcccccc}
\hline & Mean & Rank & Std & Median & \multicolumn{2}{c}{$95 \% \mathrm{Cr}^{\mathrm{c}}$} \\
\hline a[eggs] & 7.8 & 1 & 5.1 & 6.4 & 2.7 & 21.5 \\
a[turkeys] & 0.2 & 2 & 0.2 & 0.2 & 0.1 & 0.8 \\
a[chickens] & 0.0015 & 3 & 0.0024 & 0.0006 & 0.00001 & 0.008 \\
a[pigs] & 0.0002 & 4 & 0.0001 & 0.0002 & 0.00004 & 0.0005 \\
q[Infantis] & 81.3 & 1 & 15.0 & 84.9 & 44.6 & 99.4 \\
q[Give] & 62.6 & 2 & 24.6 & 65.6 & 12.9 & 98.4 \\
q[Newport] & 61.4 & 3 & 25.2 & 64.2 & 13.9 & 98.4 \\
q[Enteritidis] & 57.7 & 4 & 22.5 & 57.1 & 17.7 & 97.2 \\
q[Typhimurium] & 54.0 & 5 & 21.2 & 50.8 & 19.8 & 96.3 \\
q[Corvallis] & 49.5 & 6 & 26.1 & 47.9 & 6.7 & 96.6 \\
q[Johannesburg] & 49.4 & 7 & 26.1 & 47.8 & 6.7 & 96.6 \\
q[Braenderup] & 48.8 & 8 & 24.9 & 46.5 & 8.5 & 95.9 \\
q[London] & 21.1 & 9 & 19.1 & 14.9 & 1.7 & 76.5 \\
q[Schwarzengrund] & 17.5 & 10 & 17.4 & 11.5 & 0.9 & 67.8 \\
q[Heidelberg] & 17.4 & 11 & 18.0 & 11.0 & 0.8 & 69.8 \\
q[Saintpaul] & 16.2 & 12 & 13.4 & 12.5 & 1.8 & 52.4 \\
q[Panama] & 10.7 & 13 & 6.0 & 9.5 & 2.6 & 25.6 \\
q[Agona] & 3.4 & 14 & 2.7 & 2.7 & 0.4 & 10.5 \\
q[Derby] & 2.8 & 15 & 3.9 & 1.7 & 0.2 & 11.9 \\
\hline
\end{tabular}

${ }^{a}$ Values of $\left\{a_{j}\right\}$ are in $10^{-5}$

${ }^{\mathrm{b}}$ Standard deviation

${ }^{\mathrm{C}}$ Credibility interval 


\section{Figure legends}

718 Figure 1. Diagram illustrating the build of the final dataset for source attribution. Dark green, blue, pink, 719 and orange blocks represent datasets. The light green block represents surrogate data from the outbreak 720 dataset to complement the reservoir level, even at the exposure level. The white block represents data 721 not included in the final dataset due to a lack of subtyping.

722

723 Figure 2. The distribution of the major serovars implicated in human salmonellosis outbreak-related cases 724 from 2005 to 2015.

725

726 Figure 3. Graph of observed and expected values of the number of cases reported according to Salmonella 727 serovar.

728

729 Figure 4. Proportion of salmonellosis outbreaks attributed to foods in Rio Grande do Sul, in the triennia $730 \quad$ 2006-2008 and 2009-2011, and quadrennium 2012-2015.

731

732

Figure 5. Proportion of salmonellosis outbreaks attributed to foods in Rio Grande do Sul regions.

Figure 6. Prior prevalence $(P j)$ of Salmonella in simple foods (mean and $95 \%$ credibility interval) in Rio 735 Grande do Sul. 
738 Supplementary Material S1. Tables (A-D)

739 Table A. Values of the quantities of sources available for consumption in RS during the study period (dos Santos et 740 al., 2012).

\begin{tabular}{|c|c|c|c|c|}
\hline Source/ State ${ }^{a}$ & Amount (heads/ dozens) & Mean weight $(\mathrm{kg})$ & Yield & Total (thousand t) \\
\hline Pork/RS & $5,729,720$ & 100 & 0.72 & 412.5 \\
\hline Broilers/RS & $127,724,419$ & 5 & 0.83 & 530.0 \\
\hline Turkeys/RS & 57,421 & 7 & 0.75 & 0.3 \\
\hline Eggs/RS & 300,738 & 0.684 & 0.9 & 0.1 \\
\hline Pork/SC & $29,372,467$ & 100 & 0.72 & 2114.8 \\
\hline Broilers/SC & $22,848,963$ & 5 & 0.83 & 94.8 \\
\hline Broilers/PR & $19,962,358$ & 5 & 0.83 & 82.8 \\
\hline Broilers/GO & 818,687 & 5 & 0.83 & 3.3 \\
\hline Turkeys/SC; PR; GO & 297,881 & 7 & 0.75 & 1.5 \\
\hline
\end{tabular}

${ }^{a}$ GO: Goiás; PR: Paraná; RS: Rio Grande do Sul; SC: Santa Catarina 
743 Table B. Relative proportion of Salmonella spp. found in poultry and turkey farms in the states of Goiás, Paraná, 744 Santa Catarina, and Rio Grande do Sul

\begin{tabular}{lll}
\hline \hline Serovar & Broiler & Turkey \\
\hline Enteritidis & $\mathbf{3 0 . 8} \%$ & $0.6 \%$ \\
Schwarzengrund/Bredeney & $\mathbf{8 . 1 \%}$ & $0.6 \%$ \\
Mbandaka & $\mathbf{6 . 0 \%}$ & $\mathbf{2 . 5 \%}$ \\
Heidelberg & $\mathbf{4 . 5 \%}$ & $0.6 \%$ \\
Infantis & $\mathbf{4 . 1 \%}$ & $\mathbf{2 . 5 \%}$ \\
Agona & $\mathbf{3 . 6 \%}$ & $\mathbf{3 5 . 7} \%$ \\
Muenchen & $\mathbf{3 . 6 \%}$ & $0.0 \%$ \\
Saintpaul & $\mathbf{3 . 3} \%$ & $\mathbf{5 . 1 \%}$ \\
Typhimurium & $\mathbf{2 . 9 \%}$ & $\mathbf{2 . 5 \%}$ \\
Oranienburg & $\mathbf{2 . 6 \%}$ & $\mathbf{5 . 7} \%$ \\
Newport & $2.4 \%$ & $\mathbf{5 . 1 \%}$ \\
Senftenberg & $2.1 \%$ & $\mathbf{9 . 6 \%}$ \\
Typhimurium/4,[5],12:i:- & $2.1 \%$ & $\mathbf{4 . 5 \%}$ \\
Minnesota & $2.1 \%$ & $0.6 \%$ \\
Lille & $1.9 \%$ & $0.6 \%$ \\
Brackenridge & $1.7 \%$ & $0.0 \%$ \\
Poona & $1.7 \%$ & $0.0 \%$ \\
Worthington & $1.4 \%$ & $1.9 \%$ \\
Derby & $0.0 \%$ & \\
Anatum & & $1.9 \%$ \\
Javiana & $1.0 \%$ & \\
Hadar & & \\
\hline \hline
\end{tabular}


747 Table C. Exploratory study by MAPA on the relative proportion of Salmonella spp. found in pig farms in Rio Grande 748 do Sul.

\begin{tabular}{lr}
\hline Serovar & Proportion \\
\hline Anatum & $2.7 \%$ \\
Derby & $10.3 \%$ \\
Give & $0.7 \%$ \\
Infantis & $1.4 \%$ \\
Lexington & $0.7 \%$ \\
Ohio & $1.4 \%$ \\
Oslo & $2.1 \%$ \\
Panama & $4.8 \%$ \\
Paratyphi B & $0.7 \%$ \\
Rissen & $0.7 \%$ \\
Typhimurium & $22.6 \%$ \\
$4,[5], 12: i:-$ & $14.4 \%$ \\
Enteritidis & $0.7 \%$ \\
Manhattan & $0.7 \%$ \\
Rubislaw & $1.4 \%$ \\
Javiana & $0.7 \%$ \\
Not identified & $34.2 \%$ \\
\hline \hline
\end{tabular}


751 Table D. Summary of studies used to estimate the proportion of Salmonella serovars in pigs reared in Santa Catarina.

752

\begin{tabular}{lrrl}
\hline \hline Serovars & $\begin{array}{l}\text { Sample } \\
\text { (carcasses) }\end{array}$ & Proportion & Reference \\
\hline Derby & 109 & $11.9 \%$ & da Silva et al. (2012) \\
Derby & 98 & $2.0 \%$ & Kich et al. (2011) \\
Derby & 252 & $7.1 \%$ & Pissetti et al. (2012) \\
Infantis & 252 & $2.4 \%$ & Pissetti et al. (2012) \\
London & 252 & $0.8 \%$ & Pissetti et al. (2012) \\
Mbandaka & 252 & $0.4 \%$ & Pissetti et al. (2012) \\
O:4,5 (B) & 98 & $6.1 \%$ & Kich et al. (2011) \\
O:4,5 (B) & 252 & $0.4 \%$ & Pissetti et al. (2012) \\
O:4,5:-:1.2 (Stanley) & 98 & $1.0 \%$ & Kich et al. (2011) \\
O:6,7:r:- (Infantis) & 252 & $0.4 \%$ & Pissetti et al. (2012) \\
Ohio & 252 & $0.4 \%$ & Pissetti et al. (2012) \\
Panama & 109 & $1.8 \%$ & da Silva et al. (2012) \\
Panama & 98 & $4.1 \%$ & Kich et al. (2011) \\
Panama & 252 & $0.4 \%$ & Pissetti et al. (2012) \\
Typhimurium & 109 & $16.5 \%$ & da Silva et al. (2012) \\
Typhimurium & 98 & $11.2 \%$ & Kich et al. (2011) \\
Typhimurium & 252 & $17.5 \%$ & Pissetti et al. (2012) \\
\hline \hline
\end{tabular}

753

754 
756

757

758

759

760

761

762

763

764

765

766

767

768

769

770

771

772

774 + lambdai[i] <- sum(lambdaij[i, 1:nsource $]$ )

$775+\}$

$776+$

$777+$ for(j in 1:nsource $)\{$

$778+$ lambdaj[j]<-sum(lambdaij $[1: 15, j])$

$779+\}$

$780+\mathrm{a}[1] \sim \operatorname{dunif}(0,100)$

$781+\mathrm{a}[2] \sim \operatorname{dunif}(0,100)$

$782+a[3] \sim \operatorname{dunif}(0,100)$

$783+a[4] \sim \operatorname{dunif}(0,100)$

$784+a[5]<-a[1]$

$785+a[6]<-a[2]$

$786+a[7]<-a[2]$

$787+a[8]<-a[2]$

$788+a[9]<-a[3]$

$789+$

$790+q[1] \sim \operatorname{dunif}(0,100)$

$791+q[2] \sim$ dunif $(0,100)$

$792+q[3] \sim \operatorname{dunif}(0,100)$

$793+q[4] \sim \operatorname{dunif}(0,100)$

$794+q[5] \sim \operatorname{dunif}(0,100)$

$795+q[6] \sim \operatorname{dunif}(0,100)$

$796+q[7] \sim \operatorname{dunif}(0,100)$

$797+q[8] \sim \operatorname{dunif}(0,100)$

$798+q[9] \sim$ dunif $(0,100)$

$799+\mathrm{q}[10] \sim \operatorname{dunif}(0,100)$

$800+q[11]^{\sim}$ dunif $(0,100)$

$801+q[12]^{\sim}$ dunif $(0,100)$

$802+q[13] \sim \operatorname{dunif}(0,100)$ 


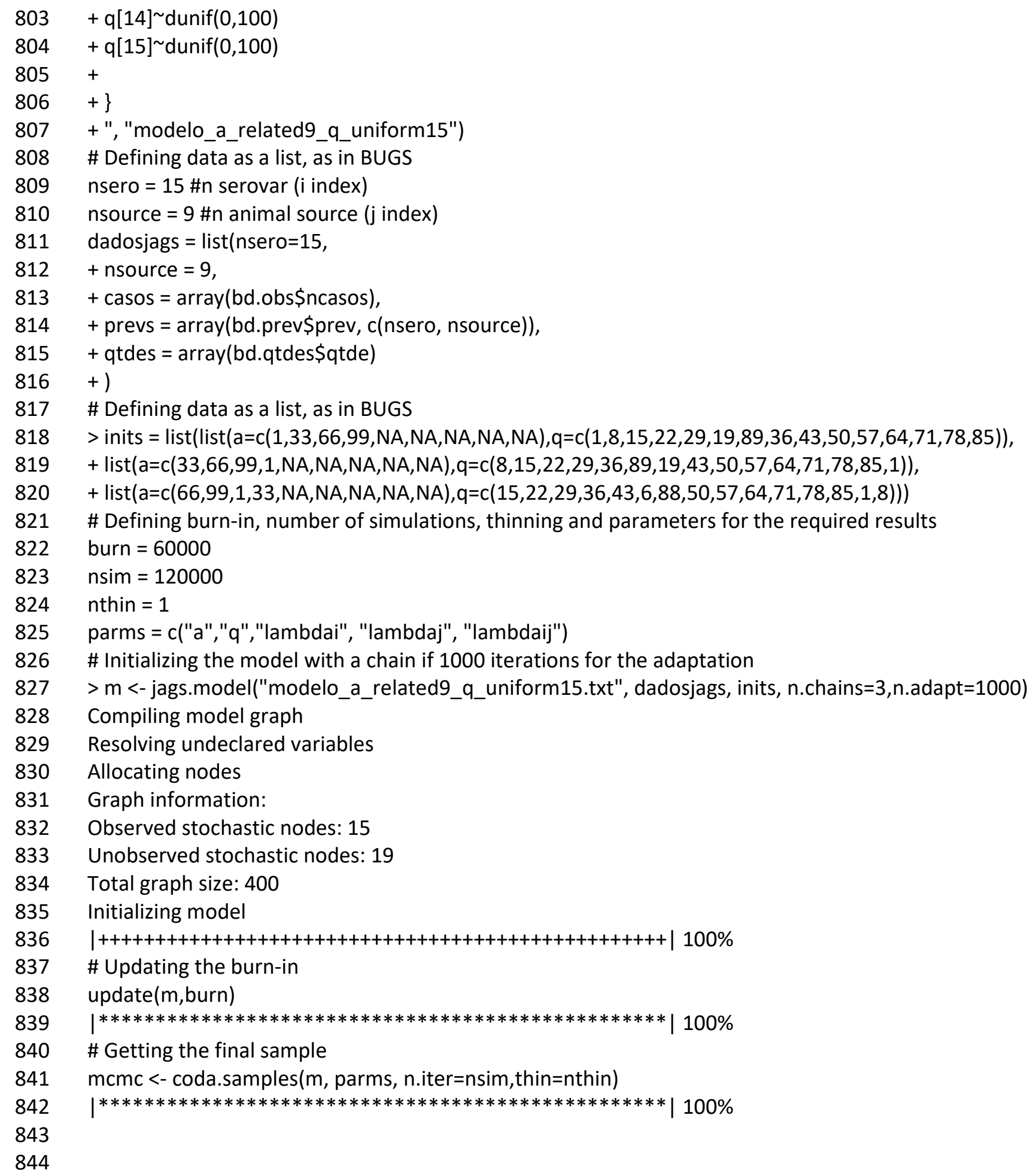


845 Code B. Salmonella source attribution model using outbreak data

846

$847 \quad$ \# model for Salmonella enterica and 12 sources (including water): periods' sub-analysis

848 \# data set: first all simple foods (o in s1: S)

849 \# complex foods (o in $1: \mathrm{C}$ )

850

851

852 \# variables:

853 \# out[i] = outbreak per pathogen

$854 \quad \# \mathrm{~s}=$ outbreak observation in simple foods

$855 \quad \# \mathrm{i}=$ outbreak observation in complex foods

$856 \quad \# \mathrm{j}=$ source

$857 \quad$ \# PriorS = prior knowledge we gain from simple food outbreaks for each source

$858 \quad \# F=$ presence or abasense of source $\mathrm{j}$ in complex food $\mathrm{f}$, matrix

859 \#sources:

860 \#1Eggs2Dairy3GoatMilk4Meat5Poultry6Chicken7Ducks8Turkey9Beef10Pork11Lamb12Mutton13Game14F 861 ruitsNuts15Vegetables16GrainsBeans17OilsSugar18Seafood19Water

862

863

864

\#1Beef2Pork3Poultry4Meat5Dairy6Eggs7Seafood8FruitsNuts9GrainsBeans10Vegetables11OilSugar12 Water

865

866 model

867\{

868

869

870

871

872 propukAA <- unknownAA * 100/ totaloutbreaksAA

873 propukBB <- unknownBB * 100/ totaloutbreaksBB

874 propukCC <- unknownCC * 100/ totaloutbreaksCC

875

876

877

878 \# Variable to estimate attribution of complex foods, separated loop (out of order) 
880

$881 \quad \# 1^{\text {st }}$ triennium

882 for (i in AAc1:AAc2 ) \{

$883 \mathrm{~b}[\mathrm{i}]<-\operatorname{sum}(\mathrm{a}[1: 12, \mathrm{i}])$

884

$885\}$

886

$887 \quad \# 2^{\text {nd }}$ triennium

888 for (i in BBc1:BBc2 ) \{

$889 b[i]<-\operatorname{sum}(a[1: 12, i])$

890

$891\}$

892

$893 \quad \# 3^{\text {rd }}$ triennium

894 for (i in CCc1:CCc2)\{

$895 \quad b[i]<-\operatorname{sum}(a[1: 12, i])$

896

$897\}$

898

899 for $(\mathrm{j}$ in 1:12) \{

900

901 \# PRIOR

902

903

904 sourceS[j] <- sum(S[j,1:totalS])

905

906

$907 \quad z[j]<-$ sourceS $[j]+1$

$908 \quad y[j]<-$ totalS - sourceS $[j]+1$

909 PriorS[j] dbeta $(z[j], y[j])$

910 
913 \# BY YEAR

914

915

$916 \quad \# 1^{\text {st }}$ triennium

917

918 \# SIMPLE FOODS

919 sourceSAA[j] <- sum(F[j, AAs1:AAs2])

920

921 \#COMPLEX FOODS

922 for (i in AAc1:AAc2) \{

923

$924 a \mathrm{a}[\mathrm{j}, \mathrm{i}]<-$ PriorS $[\mathrm{j}]^{\star} \mathrm{F}[\mathrm{j}, \mathrm{i}]$

$925 \mathrm{c}[\mathrm{j}, \mathrm{i}]<-\mathrm{a}[\mathrm{j}, \mathrm{i}] / \mathrm{b}[\mathrm{i}]$

$926 \mathrm{~d}[\mathrm{j}, \mathrm{i}]<-\mathrm{c}[\mathrm{j}, \mathrm{i}]^{*}$ PriorSU[j]

927

$928\}$

929

$930 \quad \#^{\text {nd }}$ triennium

931

932 \# SIMPLE FOODS

933 sourceSBB[j] <- sum(F[j, BBs1:BBs2])

934

935 \#COMPLEX FOODS

936 for (i in BBc1:BBc2) \{

$937 \mathrm{a}[\mathrm{j}, \mathrm{i}]<-$ PriorS[j] $\mathrm{F}[\mathrm{j}, \mathrm{i}]$

$938 \quad c[j, i]<-a[j, i] / b[i]$

$939 d[j, i]<-c[j, i]^{*}$ PriorSU[j]

940

$941\}$

942 


\section{$943 \quad \# 3^{\text {rd }}$ triennium}

944

945 \# SIMPLE FOODS

946 sourceSCC[j] <- sum(F[j, CCs1:CCs2])

947

948 \#COMPLEX FOODS

949 for (i in CCc1:CCc2) \{

$950 \mathrm{a}[\mathrm{j}, \mathrm{i}]<-$ PriorS[j]* $\mathrm{F}[\mathrm{j}, \mathrm{i}]$

$951 \quad c[j, i]<-a[j, i] / b[i]$

$952 d[j, i]<-c[j, i]^{*}$ PriorSU[j]

953

$954\}$

955

956

957 sourceCAA[j] <- sum(c[j,AAc1:AAc2])

958 sourceCBB[j] <- sum(c[j,BBc1:BBc2])

959 sourceCCC[j] <- sum(c[j,CCc1:CCc2])

960

961

962 TotalAA[j] <- sourceSAA[j] + sourceCAA[j]

963 TotalBB[j] <- sourceSBB[j] + sourceCBB[j]

964 TotalCC[j] <- sourceSCC[j] + sourceCCC[j]

965

966

967 AttribAA[j] <- $($ TotalAA[j] * $100 /$ totaloutbreaksAA $)$

968 AttribBB[j] <- $($ TotalBB[j] * $100 /$ totaloutbreaksBB $)$

969 AttribCC[j] <- $($ TotalCC[j] * $100 /$ totaloutbreaksCC)

970

$971 \quad\}$

972 PriorSU[1]<-1

973 PriorSU[2]<-1

974 PriorSU[3]<-1 


$\begin{array}{ll}975 & \text { PriorSU[4]<-1 } \\ 976 & \text { PriorSU[5]<-1 } \\ 977 & \text { PriorSU[6]<-1 } \\ 978 & \text { PriorSU[7]<-0 } \\ 979 & \text { PriorSU[8]<-0 } \\ 980 & \text { PriorSU[9]<-1 } \\ 981 & \text { PriorSU[10]<-1 } \\ 982 & \text { PriorSU[11]<-0 } \\ 983 & \text { PriorSU[12]<-1 } \\ 984 & \quad\}\end{array}$

985

986

987 \# DATA

988

989

990 list(

991

$992 \mathrm{AAs} 1=1$,

993 AAs2 $=12$,

994 AAc1 $=13$,

995 AAc2 $=151$,

996

997

998 BBs1 $=184$,

999 BBs2 = 189 ,

$1000 \mathrm{BBc} 1=190$,

1001 BBc2 = 244,

1002

1003

1004 CCs1 = 259,

1005 CCs2 $=263$,

1006 CCc1 = 264, 


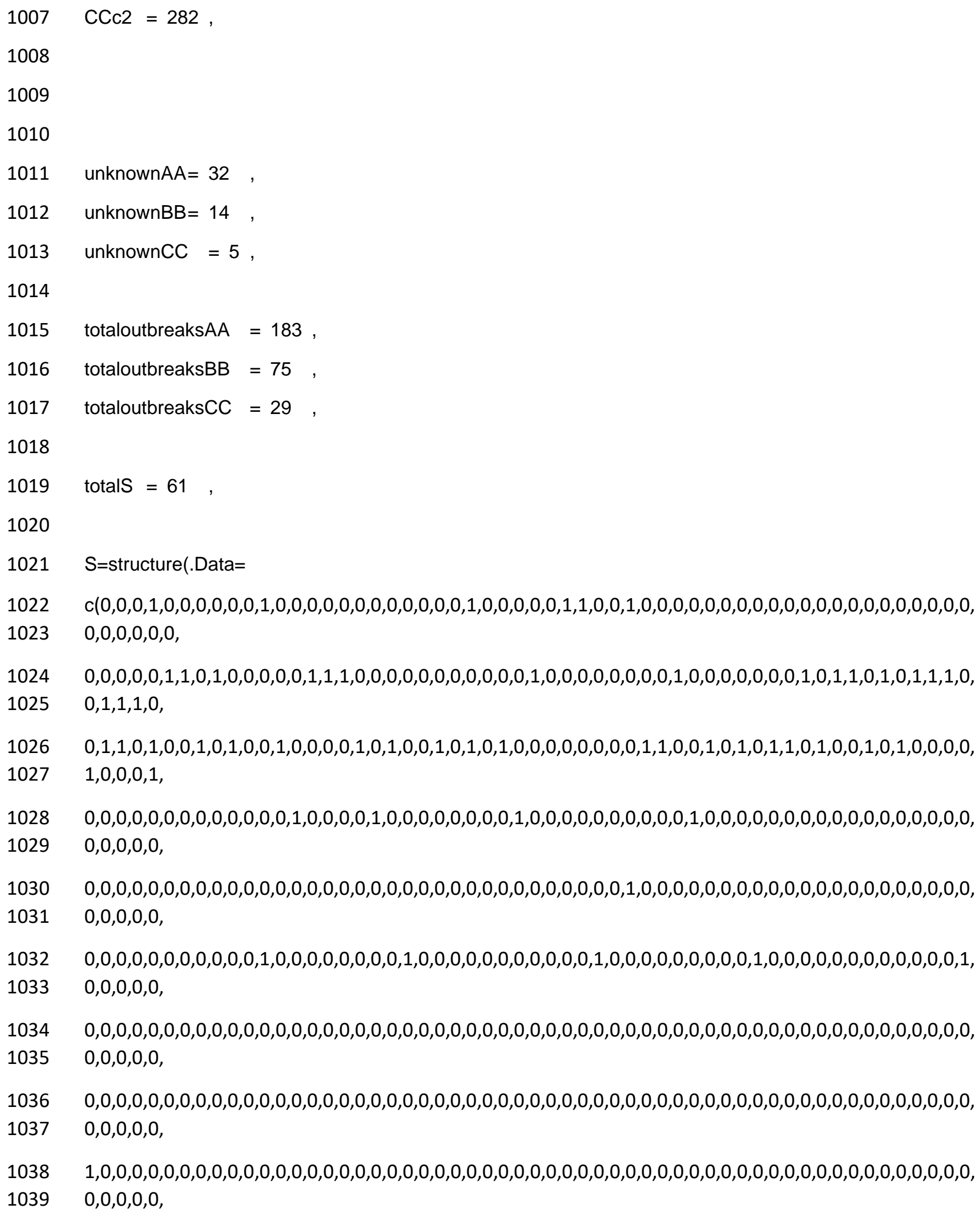


1040

1041

1042

1043

1044

1045

1046

1047

1048

1049

1050

1051

1052

1053

1054

1055

1056

1057

1058

1059

1060

1061

1062

1063

1064

1065

1066

1067

1068

1069

1070

1071

1072

1073

1074

1075

1076
$0,0,0,0,0,0,0,0,0,0,0,0,0,0,0,0,0,0,0,0,0,1,0,0,0,0,0,0,0,0,0,1,0,0,0,0,0,0,0,0,1,0,0,0,0,0,0,0,0,0,0,0,0,0,0,0$, $0,0,0,0,0$,

$0,0,0,0,0,0,0,0,0,0,0,0,0,0,0,0,0,0,0,0,0,0,0,0,0,0,0,0,0,0,0,0,0,0,0,0,0,0,0,0,0,0,0,0,0,0,0,0,0,0,0,0,0,0,0,0$, $0,0,0,0,0$,

$0,0,0,0,0,0,0,0,0,0,0,0,0,0,0,0,0,0,0,0,0,0,0,0,0,1,0,0,0,0,0,0,0,0,0,0,0,0,0,0,0,0,0,0,0,0,0,0,0,0,0,0,0,0,0,0$, $0,0,0,0,0$

),. $\operatorname{Dim}=c(12,61))$,

$\mathrm{F}=$ structure $($. Data $=$

$c($

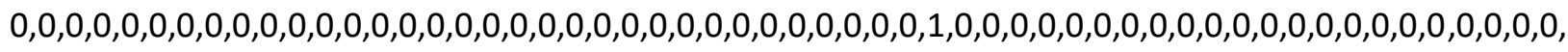
$0,0,0,0,0,0,0,0,0,0,0,0,0,0,0,0,0,0,0,0,0,0,0,0,0,0,0,0,0,0,0,0,0,0,0,0,0,0,0,0,0,0,0,0,0,0,0,0,0,0,0,0,0,0,0,0$, $0,0,0,0,0,0,0,0,0,0,0,0,0,0,0,0,0,0,0,0,0,0,0,0,0,0,1,0,0,0,0,0,0,0,0,0,0,0,0,0,0,0,0,0,0,1,0,0,0,0,0,1,0,0,0,1$, $1,0,1,0,0,0,0,0,0,0,0,0,0,0,0,0,0,0,0,0,0,0,0,0,1,0,0,0,0,0,0,0,0,0,0,0,0,0,0,0,1,0,0,0,0,0,0,0,0,0,0,0,0,0,0,0$, $0,0,0,0,0,0,0,0,0,0,0,0,0,0,0,0,0,0,0,0,0,0,0,0,1,0,0,0,0,0,1,0,0,0,0,0,0,0,0,1,0,0,0,0,0,0,0,0,0,0,0,0,0,0,0,0$, $0,0,0,0,0,0,0$

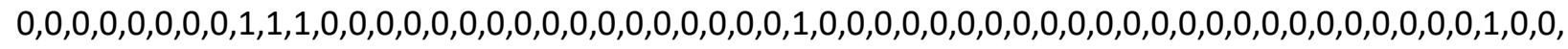
0, $0,0,0,0,0,0,0,0,0,0,0,0,0,0,0,0,0,0,0,0,0,0,0,0,0,0,0,0,0,0,0,0,0,0,0,0,0,0,0,0,0,0,0,0,0,0,0,0,0,0,0,0,0,0,0$, $0,0,0,0,0,0,0,0,0,0,0,0,0,0,0,0,0,0,0,0,0,0,0,0,0,0,0,0,0,0,0,0,0,0,0,0,0,0,0,0,0,0,1,0,0,0,0,0,0,0,0,0,0,0,0,0$,

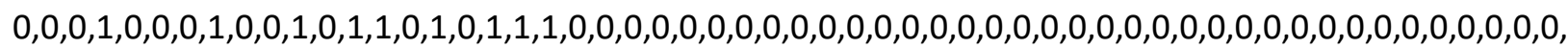
$0,0,0,0,0,0,0,0,0,0,0,0,1,0,0,0,0,0,0,0,0,0,0,0,0,0,0,0,0,0,0,0,0,0,1,0,1,1,0,0,0,0,0,1,0,0,0,0,0,0,0,1,0,0,0,0$, $0,0,0,0,0,0,0$

$0,1,0,1,1,0,1,1,0,0,0,1,0,0,0,0,1,0,0,0,0,0,0,0,0,0,0,0,0,0,0,0,0,0,0,0,0,0,0,0,0,0,0,0,0,0,0,0,0,0,0,0,0,0,0,0$, $0,0,0,0,0,0,0,0,0,0,1,0,0,0,0,0,0,0,0,0,0,0,0,0,0,0,1,0,0,0,0,0,0,0,0,0,0,0,0,0,0,0,0,0,0,0,0,0,0,0,0,0,1,1,0,0$, $0,0,0,0,0,0,0,0,0,0,0,0,0,0,0,0,0,0,0,0,0,0,0,0,0,0,0,0,0,0,0,0,0,0,0,0,0,0,0,0,1,1,0,1,1,0,1,0,0,0,0,0,0,0,1,0$, $0,0,0,0,1,1,1,0,1,0,0,0,0,0,0,0,1,0,0,0,0,0,0,0,0,0,0,1,0,0,0,0,0,0,0,0,0,0,0,0,0,0,0,0,0,0,0,0,0,0,0,0,0,0,0,0$, $0,0,0,0,0,0,0,0,0,0,0,0,0,0,0,0,0,0,0,0,1,0,0,0,0,0,0,1,0,0,0,0,1,0,0,1,0,0,1,0,0,1,0,0,0,0,0,0,0,0,0,0,0,0,0,0$, $0,0,0,0,0,0,1$

1, $0,0,0,0,0,0,0,0,0,0,0,0,0,0,0,0,0,0,0,0,0,0,0,1,0,0,0,0,0,0,0,0,0,0,0,0,0,0,0,0,0,0,0,0,0,0,0,0,0,0,0,1,0,0,0$, $0,0,0,0,0,0,0,0,0,0,0,0,0,0,0,0,0,0,0,0,0,0,0,0,0,0,0,0,0,0,0,0,0,0,0,0,0,0,0,0,0,0,0,0,0,0,0,0,0,0,0,0,0,0,0,0$, $0,0,0,0,0,0,0,1,0,0,0,0,0,0,0,0,0,0,0,0,0,0,0,1,0,0,0,0,0,0,0,0,0,0,0,0,0,0,1,0,0,0,0,0,0,0,0,0,0,0,1,0,1,1,0,0$, $0,0,0,0,0,0,0,0,0,0,0,0,0,0,0,0,0,0,0,0,0,0,0,0,0,0,0,0,0,1,0,0,0,0,0,0,0,0,0,0,0,0,0,0,0,0,0,0,0,0,0,0,0,0,0,0$, $0,0,0,1,0,1,0,0,0,0,0,0,0,0,0,1,1,1,0,0,0,0,0,1,0,0,0,0,0,0,0,0,0,0,0,0,0,0,0,0,1,0,0,0,0,0,1,0,0,0,0,0,0,0,0,0$, $0,0,0,1,1,1,0$

$0,0,0,0,0,0,0,0,0,0,0,0,0,0,0,1,1,0,0,0,0,0,0,0,1,0,0,1,0,0,0,0,1,0,0,0,0,0,0,0,0,0,0,1,0,0,0,0,0,0,0,0,1,0,0,0$, $0,0,1,0,0,0,0,0,0,0,0,0,0,0,0,0,0,1,0,0,0,0,0,0,0,1,1,0,0,0,0,0,0,0,0,1,1,0,0,0,0,1,0,0,0,0,0,0,0,0,1,0,0,0,0,0$, $0,0,0,1,1,0,0,0,0,0,0,1,0,0,0,0,1,0,0,0,0,0,0,0,0,0,1,0,0,0,0,0,0,1,0,0,0,0,1,1,0,0,0,0,0,0,0,1,0,0,0,1,0,0,0,0$, 
1077

1078

1079

1080

1081

1082

1083

1084

1085

1086

1087

1088

1089

1090

1091

1092

1093

1094

1095

1096

1097

1098

1099

1100

1101

1102

1103

1104

1105

1106

1107

1108

1109

1110

1111

1112

1113
$0,0,0,0,0,0,0,0,0,1,0,0,0,0,0,0,0,0,0,0,0,0,0,0,0,1,0,0,0,0,0,0,0,0,0,0,0,1,1,0,1,0,0,0,0,0,0,0,0,0,1,0,0,0,0,0$, $0,0,0,1,0,1,0,1,1,0,0,0,1,0,1,0,0,1,1,0,0,1,0,0,0,0,0,0,1,0,0,0,0,0,0,0,0,0,0,1,1,0,0,0,0,1,1,0,0,0,0,1,1,0,1,0$, $1,0,0,1,1,1,0$

$0,0,0,0,0,1,0,0,0,0,0,0,1,1,1,1,1,1,1,1,1,1,1,1,1,1,1,1,0,1,1,1,1,1,1,1,1,1,1,1,1,1,1,0,1,1,1,1,1,1,1,1,1,0,1,1$, $1,1,1,1,1,1,1,1,1,1,1,1,1,1,1,1,1,0,1,1,1,1,1,1,1,1,0,1,1,1,1,1,1,1,1,1,0,1,1,1,1,0,1,1,1,1,1,1,1,1,1,1,1,1,1,1$, $1,1,1,1,1,1,1,1,1,1,1,0,1,1,1,1,1,1,1,1,1,1,1,1,1,1,0,1,1,1,1,1,1,1,1,1,1,1,0,1,1,1,1,0,1,1,1,1,0,0,1,0,1,1,1,1$, $1,0,1,1,1,1,1,1,1,0,1,0,1,1,0,0,0,0,0,0,1,1,1,1,1,1,1,1,1,0,1,1,1,1,1,1,1,0,0,1,1,1,1,1,1,1,1,1,1,1,1,1,1,1,1,1$, $1,1,1,1,1,1,1,1,1,1,1,1,0,1,1,0,0,1,1,1,1,1,0,1,1,0,0,1,1,1,1,0,1,0,0,0,0,0,0,0,0,0,1,1,1,1,1,1,1,1,1,1,0,1,1,1$, $1,1,0,0,0,0,1$

$0,0,0,0,0,0,0,0,0,0,0,0,0,0,0,0,0,0,0,0,0,0,0,0,0,0,0,0,0,0,0,0,0,0,0,0,0,0,0,0,0,0,0,0,0,0,0,0,0,0,0,0,0,0,0,0$, $0,0,0,0,0,0,0,0,0,0,0,0,0,0,0,0,0,0,0,0,0,0,0,0,0,0,0,0,0,0,0,0,0,0,0,0,0,0,0,0,0,0,0,0,0,0,0,0,0,0,0,0,0,0,0,0$, $0,0,0,0,0,0,0,0,0,0,0,0,0,0,0,0,0,0,0,0,0,0,0,0,0,0,0,0,0,0,0,0,0,0,0,0,0,0,0,0,0,0,0,0,0,0,0,0,0,0,0,0,0,0,0,0$, $0,0,0,0,0,0,0,0,0,0,0,0,0,0,0,0,0,0,0,0,0,0,0,0,0,0,0,0,0,0,0,0,0,0,0,0,0,0,0,0,0,0,0,0,0,0,0,0,0,0,0,0,0,0,0,0$, $0,0,0,0,0,0,0,0,0,0,0,0,0,0,0,0,0,0,0,0,0,0,0,0,0,0,0,0,0,0,0,0,0,0,0,0,0,0,0,0,0,0,0,0,0,0,0,0,0,0,0,0,0,0,0,0$, $0,0,0,0,0,0,0$

$0,0,0,0,0,0,0,0,0,0,0,0,0,0,0,1,0,0,0,0,0,0,0,0,0,0,0,0,0,0,0,0,0,0,0,0,0,0,0,0,0,0,0,0,0,0,0,0,0,0,0,0,0,0,0,0$, $0,0,1,0,0,0,0,0,0,0,0,0,0,0,0,0,0,1,0,0,0,0,0,0,0,0,0,0,0,0,0,0,0,0,0,0,0,0,0,0,0,0,0,0,0,0,0,0,0,0,0,0,0,0,0,0$, $0,0,0,0,1,0,0,0,0,0,0,0,0,0,0,0,0,0,0,0,0,0,0,0,0,0,1,0,0,0,0,0,0,0,0,0,0,0,0,0,0,0,0,0,0,0,0,0,0,0,0,0,0,0,0,0$, $0,0,0,0,0,0,0,1,0,1,0,0,0,0,0,0,0,0,0,0,0,0,0,0,0,0,0,0,0,0,0,0,0,0,0,0,0,0,0,0,0,0,0,0,0,0,0,0,0,0,0,0,0,0,0,0$, $0,0,0,0,0,0,0,0,0,0,0,0,0,0,1,0,0,0,0,0,0,0,0,0,0,0,0,0,0,0,0,0,0,0,0,0,0,0,0,0,0,0,0,0,0,0,0,0,0,0,0,0,0,0,0,0$, $0,0,0,0,0,0,0$

0, $0,0,0,0,0,0,0,0,0,0,0,1,0,0,1,1,0,0,0,0,0,0,0,1,0,0,1,0,0,0,0,0,1,0,0,0,0,0,0,0,0,0,0,0,0,0,0,0,1,0,0,1,1,0,0$, $0,0,1,0,0,0,0,0,0,0,0,0,0,0,0,1,0,0,0,0,0,0,0,0,0,1,1,0,0,0,0,0,0,0,0,0,0,0,0,0,0,0,0,1,0,0,0,0,0,0,1,0,1,1,0,0$, $0,0,0,1,1,0,0,1,0,0,0,1,0,0,0,0,1,0,0,0,0,0,0,1,0,0,0,0,0,0,0,0,0,1,0,0,0,0,1,1,1,1,0,1,0,1,0,1,0,0,0,1,0,0,0,0$, $0,0,0,0,0,1,0,0,0,0,0,0,0,0,0,0,0,0,0,0,0,0,0,0,1,1,0,1,0,1,0,0,0,0,0,0,0,1,0,0,1,0,0,0,0,0,0,0,0,0,1,0,0,0,0,0$, $0,0,0,1,0,1,0,0,1,0,0,0,1,0,1,1,1,1,1,0,0,1,0,1,0,0,0,0,1,0,0,0,1,0,0,0,0,0,0,1,1,0,0,1,0,0,1,0,0,0,0,1,1,0,1,0$, $0,0,0,1,1,1,0$

0,0,1,0,0,0,0,0,0,0,0,0,1,1,0,0,1,1,1,1,1,1,1,1,1,1,1,0,0,1,1,1,0,0,1,1,1,1,1,0,1,1,1,0,1,1,1,1,1,1,1,1,1,1,1,1, $1,1,0,1,1,1,1,1,1,1,1,1,1,1,1,0,1,0,1,1,1,1,1,1,1,0,0,1,1,1,1,1,1,1,1,0,1,1,1,1,1,1,1,1,1,1,1,1,1,1,0,1,1,1,1,1$, $1,1,1,0,0,1,1,0,1,1,1,0,1,1,1,1,0,1,1,1,1,1,1,0,1,1,1,1,1,1,1,1,1,0,1,1,1,0,1,1,1,1,1,0,1,1,1,1,0,0,1,1,1,1,1,1$, $1,0,1,1,1,1,1,1,1,1,1,0,1,1,0,0,0,0,0,0,0,1,1,1,1,0,1,0,1,0,1,1,1,1,1,1,1,0,0,1,1,1,1,1,1,1,0,1,1,1,0,1,1,1,1,1$, $1,1,1,1,1,1,1,1,0,1,1,1,1,1,0,1,1,0,0,1,1,1,0,1,1,0,0,1,1,1,1,0,1,0,0,0,0,0,0,1,1,0,1,1,1,1,1,1,1,1,1,1,1,1,0,1$, $0,1,0,1,1,1,1$

0,0,0,0,0,0,0,0,0,0,0,0,1,1,1,1,0,1,1,1,1,1,1,1,1,1,1,1,1,1,1,1,1,1,1,1,1,1,1,1,1,1,1,1,1,1,1,1,1,1,1,1,1,1,1,1, $1,1,1,1,1,1,1,1,1,1,1,1,1,1,1,1,1,1,1,1,1,1,1,1,1,1,0,1,1,1,1,1,1,1,1,1,1,1,1,1,1,1,1,1,1,1,1,1,1,1,1,1,1,1,1,1$, 1,1,1,1,1,1,1,1,1,1,1,1,1,1,1,1,1,1,1,1,1,1,1,0,1,1,1,1,1,1,1,1,1,1,1,1,1,1,1,1,1,1,1,1,1,1,1,1,0,0,1,1,1,1,1,1, $1,0,1,1,1,1,1,1,1,1,1,0,1,1,0,0,0,0,0,0,0,1,1,1,1,1,1,1,1,0,1,1,1,1,1,1,1,1,1,1,1,1,1,1,1,1,1,1,1,1,1,1,1,1,1,1$, 
$11141,1,1,1,1,1,1,1,1,1,1,1,1,1,1,1,1,1,1,1,1,1,0,1,1,0,0,1,1,1,1,0,1,0,0,0,0,0,0,1,1,0,1,1,1,1,1,1,1,1,1,1,0,1,1,1$, $1115 \quad 1,1,0,1,0,1,1$

$11160,0,0,0,0,0,0,0,0,0,0,0,0,0,0,0,0,0,0,0,0,0,0,0,0,0,0,0,0,0,0,0,0,0,0,0,0,0,0,0,0,1,0,1,0,0,0,0,0,0,0,0,0,0,0,0$, $11170,0,0,0,0,0,0,0,0,0,0,0,0,0,0,0,0,0,0,0,0,0,0,0,0,0,0,0,0,0,0,0,0,0,0,0,0,0,0,0,0,0,0,0,0,0,0,0,0,0,0,0,0,0,0,0$, $11180,0,0,0,0,0,0,0,0,0,0,0,0,0,0,0,0,0,0,0,0,0,0,0,0,0,0,0,0,0,0,0,0,0,0,0,0,0,0,0,0,0,0,0,0,0,0,0,0,0,0,0,0,0,0,0$, $11190,0,0,0,0,0,0,0,0,0,0,0,0,0,0,0,0,0,0,0,0,0,0,0,0,0,0,0,0,0,0,0,0,0,0,0,0,0,0,0,0,0,0,0,0,0,0,0,0,0,0,0,0,0,0,0$, $11200,0,0,0,0,0,0,0,0,0,0,0,0,0,0,0,0,0,0,0,0,0,0,0,0,0,0,0,0,0,0,0,0,0,0,0,0,0,0,0,0,0,0,0,0,0,0,0,0,0,0,0,0,0,0,0$, $11210,0,0,0,0,0,0$

$1122), . \operatorname{Dim}=c(12,287)))$

1123

$1124), . \operatorname{Dim}=c(12, X X)))$

1125

1126 \# INITIAL VALUES

1127

1128 list $($ PriorS $=c(0.1,0.1,0.1,0.1,0.1,0.1,0.1,0.1,0.1,0.1,0.1,0.1))$

1129

$1130 \quad$ list $($ PriorS $=c(0.01,0.01,0.01,0.01,0.01,0.01,0.01,0.01,0.01,0.01,0.01,0.01))$

1131

1132 list $($ PriorS $=c(0.2,0.2,0.2,0.2,0.2,0.2,0.2,0.2,0.2,0.2,0.2,0.2))$

1133

1134 list $($ PriorS $=c(0.05,0.05,0.05,0.05,0.05,0.05,0.05,0.05,0.05,0.05,0.05,0.05))$

1135

1136

1137

1138

1139

1140

1141

1142

1143

1144

1145

1146

1147

1148

1149

1150

1151

1152

list $($ PriorS $=c(0.3,0.3,0.3,0.3,0.3,0.3,0.3,0.3,0.3,0.3,0.3,0.3))$ 
1153 \# model for SALMONELLA and 12 sources (including water): regions' sub-analysis

1154 \# data set: first all simple foods (o in s1: S)

1155 \# complex foods (o in c1:C)

1156

1157

1158 \# variables:

$1159 \quad$ \# out[i] = outbreak per pathogen

$1160 \quad \# \mathrm{~s}=$ outbreak observation in simple foods

$1161 \quad \# \mathrm{i}=$ outbreak observation in complex foods

$1162 \# \mathrm{j}=$ source

1163 \# PriorS = prior knowledge we gain from simple food outbreaks for each source

$1164 \quad \# \mathrm{~F}=$ presence or absence of source $\mathrm{j}$ in complex food $\mathrm{f}$, matrix

1165 \#sources:

1166 \#1Eggs2Dairy3GoatMilk4Meat5Poultry6Chicken7Ducks8Turkey9Beef10Pork11Lamb12Mutton13Game14F

1167 ruitsNuts15Vegetables16GrainsBeans17OilsSugar18Seafood19Water

1168

1169

1170

\#1Beef2Pork3Poultry4Meat5Dairy6Eggs7Seafood8FruitsNuts9GrainsBeans10Vegetables11OilSugar12 Water

1171

1172

1173 model

1174\{

1175

\section{6 \# Proportion of unknowns}

1177

1178

1179

1180

1181 propukCOC <- unknownCOC * $100 /$ totaloutbreaksCOC

1182 propukCOR <- unknownCOR * 100/totaloutbreaksCOR

1183 propukMPA <- unknownMPA * 100/ totaloutbreaksMPA

1184 propukNE <- unknownNE * 100/ totaloutbreaksNE

1185 propukNO <- unknownNO * 100/ totaloutbreaksNO 
1186 propukSE <- unknownSE * 100/ totaloutbreaksSE

1187 propukSO <- unknownSO * 100/ totaloutbreaksSO

1188

1189

1190 \# Variable to estimate attribution of complex foods, separated loop (out of order)

1191

1192 \#COC

1193 for (i in COCc1:COCc2 ) \{

$1194 \mathrm{~b}[\mathrm{i}]<-\operatorname{sum}(\mathrm{a}[1: 12, \mathrm{i}])$

1195

$1196\}$

1197

1198 \#COR

1199 for (i in CORc1:CORc2) \{

$1200 \quad b[i]<-\operatorname{sum}(a[1: 12, i])$

1201

$1202\}$

1203

1204 \#MPA

1205 for ( $\mathrm{i}$ in MPAc1:MPAc2) \{

$1206 \mathrm{~b}[\mathrm{i}]<-\operatorname{sum}(\mathrm{a}[1: 12, \mathrm{i}])$

1207

$1208\}$

1209

1210 \#NE

1211 for (i in NEc1:NEc2 ) \{

$1212 b[i]<-\operatorname{sum}(a[1: 12, i])$

1213

$1214\}$

1215

1216 \#NO

1217 for (i in NOc1:NOc2 ) \{ 
$1218 \quad b[i]<-\operatorname{sum}(a[1: 12, i])$

1219

$1220\}$

1221

1222 \#SE

1223 for (i in SEc1:SEc2) \{

$1224 b[i]<-\operatorname{sum}(a[1: 12, i])$

1225

$1226\}$

1227

1228 \#SO

1229 for (i in SOc1:SOc2) \{

$1230 \quad b[i]<-\operatorname{sum}(a[1: 12, i])$

1231

$1232\}$

1233

1234

1235

1236

1237 for $(\mathrm{j}$ in $1: 12)$ \{

1238

1239 \# PRIOR

1240

1241

1242 sourceS[j] <- sum(S[j,1:totalS])

1243

1244

$1245 \quad z[j]<-$ sourceS[j] +1

$1246 \quad y[j]<-$ totalS - sourceS[j] +1

1247 PriorS[j] dbeta(z[j], y[j])

1248

1249 
1250 \# BY REGION

1251 \#COC

1252 \# SIMPLE FOODS

1253 sourceSCOC[j] <- sum(F[j,COCs1:COCs2])

1254 \# COMPLEX FOODS

1255 for (i in COCc1:COCc2) \{

$1256 \mathrm{a}[\mathrm{j}, \mathrm{i}]<-$ PriorS[j] * F[j,i]

$1257 \mathrm{c}[\mathrm{j}, \mathrm{i}]<-\mathrm{a}[\mathrm{j}, \mathrm{i}] / \mathrm{b}[\mathrm{i}]\}$

1258 \#COR

1259 \# SIMPLE FOODS

1260 sourceSCOR[j] <- sum(F[j,CORs1:CORs2])

1261 \# COMPLEX FOODS

1262 for (i in CORc1:CORc2) \{

$1263 a[\mathrm{a}, \mathrm{i}]<-$ PriorS[j] * $\mathrm{F}[\mathrm{j}, \mathrm{i}]$

$1264 \mathrm{c}[\mathrm{j}, \mathrm{i}]<-\mathrm{a}[\mathrm{j}, \mathrm{i}] / \mathrm{b}[\mathrm{i}]\}$

1265 \#MPA

1266 \# SIMPLE FOODS

1267 sourceSMPA[j] <- sum(F[j,MPAs1:MPAs2])

1268 \# COMPLEX FOODS

1269 for (i in MPAc1:MPAc2) \{

$1270 \mathrm{a}[\mathrm{j}, \mathrm{i}]<-$ PriorS[j] * F[j,i]

$1271 \mathrm{c}[\mathrm{j}, \mathrm{i}]<-\mathrm{a}[\mathrm{j}, \mathrm{i}] / \mathrm{b}[\mathrm{i}]\}$

1272 \#NE

1273 \# SIMPLE FOODS

1274 sourceSNE[j] <- sum(F[j,NEs1:NEs2])

1275 \# COMPLEX FOODS

1276 for (i in NEc1:NEc2 ) \{

$1277 a[\mathrm{a}, \mathrm{i}]<-$ PriorS[j] * F[j,i]

$1278 \mathrm{c}[\mathrm{j}, \mathrm{i}]<-\mathrm{a}[\mathrm{j}, \mathrm{i}] / \mathrm{b}[\mathrm{i}]\}$

1279 \#NO

1280 \# SIMPLE FOODS

1281 sourceSNO[j] <- sum(F[j,NOs1:NOs2]) 
1282 \# COMPLEX FOODS

1283 for (i in NOc1:NOc2 ) \{

$1284 a[j, i]<-$ PriorS $[j]{ }^{*} F[j, i]$

$1285 \mathrm{c}[\mathrm{j}, \mathrm{i}]<-\mathrm{a}[\mathrm{j}, \mathrm{i}] / \mathrm{b}[\mathrm{i}]\}$

1286 \#SE

1287 \# SIMPLE FOODS

1288 sourceSSE[j] <- sum(F[j,SEs1:SEs2])

1289 \# COMPLEX FOODS

1290 for (i in SEc1:SEc2 ) \{

$1291 \mathrm{a}[\mathrm{j}, \mathrm{i}]<-$ PriorS[j] * $\mathrm{F}[\mathrm{j}, \mathrm{i}]$

$1292 \mathrm{c}[\mathrm{j}, \mathrm{i}]<-\mathrm{a}[\mathrm{j}, \mathrm{i}] / \mathrm{b}[\mathrm{i}]\}$

1293 \#SO

1294 \# SIMPLE FOODS

1295 sourceSSO[j] <- sum(F[j,SOs1:SOs2])

1296 \# COMPLEX FOODS

1297 for (i in SOc1:SOc2 ) \{

$1298 \mathrm{a}[\mathrm{j}, \mathrm{i}]<-$ PriorS[j] * $\mathrm{F}[\mathrm{j}, \mathrm{i}]$

$1299 \mathrm{c}[\mathrm{j}, \mathrm{i}]<-\mathrm{a}[\mathrm{j}, \mathrm{i}] / \mathrm{b}[\mathrm{i}]\}$

1300

1301 sourceCCOC[j] <- sum(c[j,COCc1:COCc2 ])

1302 sourceCCOR[j] <- sum(c[j,CORc1:CORc2])

1303 sourceCMPA[j] <- sum(c[j,MPAc1:MPAc2 ])

1304 sourceCNE[j] <- sum(c[j,NEc1:NEc2])

1305 sourceCNO[j] <- sum(c[j,NOc1:NOc2])

1306 sourceCSE[j] <- sum(c[j,SEc1:SEc2])

1307 sourceCSO[j] <- sum(c[j,SOc1:SOc2])

1308

1309 TotalCOC[j] <- sourceSCOC[j] + sourceCCOC[j]

1310 TotalCOR[j] <- sourceSCOR[j] + sourceCCOR[j]

1311 TotalMPA[j] <- sourceSMPA[j] + sourceCMPA[j]

1312 TotalNE[j] <- sourceSNE[j] + sourceCNE[j]

1313 TotalNO[j] <- sourceSNO[j] + sourceCNO[j] 
1314 TotalSE[j] <- sourceSSE[j] + sourceCSE[j]

1315 TotalSO[j] <- sourceSSO[j] + sourceCSO[j]

1316

1317 AttribCOC[j] <- $($ TotalCOC[j] * 100) / totaloutbreaksCOC

1318 AttribCOR[j] <- $($ TotalCOR[j] * 100) / totaloutbreaksCOR

1319 AttribMPA[j] <- $($ TotalMPA[j] * 100) / totaloutbreaksMPA

1320 AttribNE[j] <- $($ TotalNE[j] * 100) / totaloutbreaksNE

1321 AttribNO[j] <- (TotalNO[j] * 100) / totaloutbreaksNO

1322 AttribSE[j] <- (TotalSE[j] * 100) / totaloutbreaksSE

1323 AttribSO[j] <- $($ TotalSO[j] * 100) / totaloutbreaksSO

1324

1325

\}

1326

1327

1328 \# DATA

1329 list(

$1330 \operatorname{cocs} 1=1$,

1331 Cocs2 $=2$,

$1332 \operatorname{coc} 1=3$,

$1333 \operatorname{coc} 2=29$,

1334

1335 CORs $1=35$,

1336 CORs2 $=38$,

1337 CORc1 $=39$,

1338 CORc2 $=90$,

1339

1340 MPAs1 $=104$,

1341 MPAs2 $=120$,

1342 MPAC1 $=121$,

1343 MPAC2 $=225$,

1344

1345 NEs1 $=271$,

1346 NEs2 $=281$,

$1347 \mathrm{NEc} 1=282$, 
$1348 \mathrm{NEc2}=400$,

1349

1350 NOs1 $=446$,

1351 NOs2 $=470$,

1352 NOc1 $=471$,

1353 NOc2 $=714$,

1354

1355 SEs1 $=790$,

1356 SEs2 $=790$,

1357 SEc1 = 791 ,

1358 SEc2 $=803$,

1359

1360 SOs $1=810$,

1361 SOs2 $=810$,

1362 SOc1 $=811$,

1363 SOc2 $=815$,

1364

1365

1366

1367

1368 unknownCOC $=5$,

1369 unknownCOR $=13$,

1370 unknownMPA $=45$,

1371 unknownNE $=45$,

1372 unknownNO = 75,

1373 unknownSE $=6$

1374 unknownso = 2 ,

1375

1376 totaloutbreaksCOC $=34$,

1377 totaloutbreaksCOR $=69$,

1378 totaloutbreaksMPA $=167$,

1379 totaloutbreaksNE $=175$,

1380 totaloutbreaksNO = 344 ,

1381 totaloutbreaksSE $=20$,

1382 totaloutbreaksSO $=8$, 
1384 totals $=61$

1385

1386

1387

1388

1389

1390

1391 S=structure $($. Data $=$

$1392 \mathrm{c}(0,0,0,0,0,0,0,0,0,1,0,1,0,0,0,0,0,0,0,0,0,0,0,0,0,0,0,0,0,1,0,0,0,0,1,0,1,0,0,0,0,0,0,0,0,0,0,0,0,0,1,0,0,0,0$, $13930,0,0,0,0,0$

$13940,0,0,0,1,1,0,0,0,0,0,0,0,0,0,0,0,0,0,1,1,1,0,0,0,0,0,0,0,0,1,0,0,1,0,1,0,0,1,1,0,0,0,0,1,1,1,0,1,0,0,0,0,0,1,1$, $1395 \quad 0,1,0,0,1$

1396

1397

$0,1,1,1,0,0,1,0,0,0,1,0,1,1,1,0,0,1,1,0,0,0,1,1,0,0,1,0,0,0,0,0,1,0,0,0,0,1,0,0,1,0,1,1,0,0,0,1,0,0,0,0,1,1,0,0$, $0,0,1,0,0$

1398

1399

$0,0,0,0,0,0,0,0,0,0,0,0,0,0,0,0,1,0,0,0,0,0,0,0,1,0,0,0,0,0,0,0,0,0,0,0,0,0,0,0,0,1,0,0,0,0,0,0,0,1,0,0,0,0,0,0$, $0,0,0,0,0$

1400

$0,0,0,0,0,0,0,0,0,0,0,0,0,0,0,0,0,0,0,0,0,0,0,0,0,0,0,0,0,0,0,0,0,0,0,0,0,0,0,0,0,0,0,0,0,0,0,0,0,0,0,1,0,0,0,0$, $14010,0,0,0,0$

$14020,0,0,0,0,0,0,0,0,0,0,0,0,0,0,0,0,0,0,0,0,0,0,0,0,1,0,0,1,0,0,1,0,0,0,0,0,0,0,0,0,0,0,0,0,0,0,0,0,0,0,0,0,0,0,0$, $14031,0,0,1,0$

$14040,0,0,0,0,0,0,0,0,0,0,0,0,0,0,0,0,0,0,0,0,0,0,0,0,0,0,0,0,0,0,0,0,0,0,0,0,0,0,0,0,0,0,0,0,0,0,0,0,0,0,0,0,0,0,0$, $1405 \quad 0,0,0,0,0$

$14060,0,0,0,0,0,0,0,0,0,0,0,0,0,0,0,0,0,0,0,0,0,0,0,0,0,0,0,0,0,0,0,0,0,0,0,0,0,0,0,0,0,0,0,0,0,0,0,0,0,0,0,0,0,0,0$, $1407 \quad 0,0,0,0,0$

$14080,0,0,0,0,0,0,1,0,0,0,0,0,0,0,0,0,0,0,0,0,0,0,0,0,0,0,0,0,0,0,0,0,0,0,0,0,0,0,0,0,0,0,0,0,0,0,0,0,0,0,0,0,0,0,0$, $1409 \quad 0,0,0,0,0$

$14101,0,0,0,0,0,0,0,0,0,0,0,0,0,0,1,0,0,0,0,0,0,0,0,0,0,0,1,0,0,0,0,0,0,0,0,0,0,0,0,0,0,0,0,0,0,0,0,0,0,0,0,0,0,0,0$, $14110,0,0,0,0$

$14120,0,0,0,0,0,0,0,0,0,0,0,0,0,0,0,0,0,0,0,0,0,0,0,0,0,0,0,0,0,0,0,0,0,0,0,0,0,0,0,0,0,0,0,0,0,0,0,0,0,0,0,0,0,0,0$, $1413 \quad 0,0,0,0,0$

$14140,0,0,0,0,0,0,0,1,0,0,0,0,0,0,0,0,0,0,0,0,0,0,0,0,0,0,0,0,0,0,0,0,0,0,0,0,0,0,0,0,0,0,0,0,0,0,0,0,0,0,0,0,0,0,0$, $14150,0,0,0,0$

1416

1417

$1418), . \operatorname{Dim}=c(19,61))$,

1419 
$1420 \mathrm{~F}=$ structure $($.Data $=$

$1421 \quad c($

1422

1423

1424

1425

1426

1427

1428

1429

1430

1431

1432

1433

1434

1435

1436

1437

1438

1439

1440

1441

1442

1443

1444

1445

1446

1447

1448

1449

1450

1451

1452

1453

1454

1455

1456

1457

1458

1459

1460

1461

1462

1463

1464

1465

1466

1467

1468


$0,0,0,0,0,0,0,0,0,0,0,0,0,0,0,0,0,0,0,0,0,0,0,0,0,0,0,0,0,0,0,0,0,0,0,0,0,0,0,0,0,0,0,0,1,0,0,0,0,0,1,0,1,0,0,0$, $0,0,0,0,0,0,0,0,0,0,1,0,0,0,0,0,0,0,0,0,0,1,0,0,0,0,0,0,0,0,0,0,0,0,0,0,0,0,0,0,0,0,0,0,0,0,1,0,0,0,0,0,0,0,0,0$, $0,0,0,0,0,0,0,0,0,0,0,0,0,0,0,0,0,0,0,0,0,0,0,0,0,0,0,0,0,0,0,0,0,0,0,0,0,0,1,0,0,0,0,0,0,0,0,0,0,0,0,0,1,0,0,0$, $0,0,0,0,0,0,1,0,0,0,0,0,0,0,0,0,0,1,1,0,0,0,0,1,0,1,0,0,0,0,0,1,0,0,0,0,0,0,0,0,0,1,0,0,0,0,0,0,0,0,0,0,1,0,0,0$, $0,0,0,0,0,0,0,0,0,1,0,0,0,0,0,0,0,0,0,0,0,0,0,0,0,0,0,0,0,0,0,0,0,0,0,0,0,0,0,0,1,0,0,1,0,0,0,0,0,0,0,0,0,0,0,0$, $0,0,0,0,0,0,0,0,0,0,0,0,0,0,0,0,0,0,0,0,0,0,0,0,0,0,0,1,0,0,0,0,0,0,0,0,0,0,0,0,0,0,0,0,1,0,0,1,0,0,0,0,0,0,0,0$, $0,0,0,0,0,0,0,0,0,0,0,1,0,0,0,0,0,0,0,0,0,1,0,0,0,0,0,0,0,0,0,0,0,0,0,0,0,0,0,0,0,1,0,1,0,1,1,0,0,0,1,0,0,1,0,1$, $0,0,0,0,0,0,0,0,0,0,0,0,0,1,0,0,0,0,0,0,0,0,0,0,0,0,0,0,0,0,0,0,0,0,0,0,0,0,0,0,0,0,0,0,0,0,0,0,0,0,0,0,0,0,0,0$, $0,0,0,0,0,0,0,0,0,0,0,0,0,0,0,0,0,0,0,0,0,0,0,0,0,0,0,0,0,0,0,0,0,0,0,0,0,0,0,0,0,0,0,0,0,0,0,0,0,0,0,0,0,0,0,0$, $0,0,0,0,0,0,0,0,0,0,0,0,0,0,0,0,0,0,0,0,0,0,0,0,0,0,0,0,0,0,0,0,0,0,0,0,0,0,0,0,0,0,0,0,0,0,0,0,0,0,0,0,0,0,0,0$, $0,0,0,0,0,0,0,0,0,0,0,0,0,0,0,0,0,0,0,0,0,0,0,0,0,0,0,0,0,0,0,0,0,0,0,0,0,0,0,0,0,0,0,0,0,0,0,0,0,0,0,0,0,0,0,0$, $0,0,0,0,0,0,0,0,0,0,0,0,0,0,0,0,0,0,0,0,0,0,0,0,0,0,0,0,0,0,0,0,0,0,0,0,0,0,0,0,0,0,0,0,0,1,0,0,0,0,0,0,0,0,0,1$, $0,0,0,1,0,0,0,0,0,0,0,0,1,0,0,0,0,0,1,0,1,0,0,0,0,0,0,0,1,0,0,0,0,0,0,0,1,0,1,1,1,0,0,0,0,0,0,0,0,0,1,0,0,0,0,0$, $0,0,0,0,0,0,0,0,1,0,0,0,0,0,0,0,0,0,0,0,0,0,0,0,0,0,0,0,0,0,0,1,0$

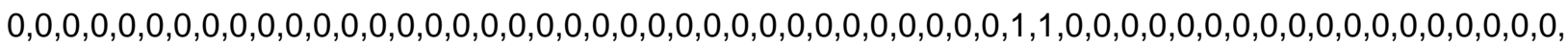
$0,0,0,0,0,0,0,0,0,0,0,0,0,0,0,0,0,0,0,0,0,0,0,0,0,0,0,0,0,0,0,0,0,0,0,0,0,1,0,0,0,0,0,1,0,0,0,0,0,0,0,0,0,0,0,0$, $0,0,0,0,1,1,1,0,0,0,0,0,0,0,0,0,0,1,0,0,0,0,0,0,0,0,0,0,0,0,0,0,0,0,0,0,0,0,0,0,0,0,0,0,0,0,0,0,0,0,0,0,0,0,0,0$, $0,0,0,0,0,0,0,0,0,0,0,0,1,0,0,0,0,0,0,0,0,0,0,0,0,0,0,0,0,0,0,0,0,0,0,0,0,0,0,0,0,0,0,0,0,0,0,0,0,0,0,0,0,0,1,0$, $0,0,0,0,0,0,0,0,0,0,0,0,0,0,1,0,0,0,0,0,0,0,0,0,1,0,0,0,0,0,0,0,0,0,1,1,0,0,0,0,0,0,0,0,0,0,0,0,0,0,0,0,0,1,0,0$, $1,0,0,0,0,0,0,0,0,0,0,0,0,0,0,0,0,0,0,0,0,0,0,0,0,0,0,0,0,0,0,0,0,0,0,0,0,0,0,0,0,0,0,0,0,0,0,0,1,0,0,0,0,0,0,0$, $0,0,0,0,0,0,0,0,0,0,0,0,0,0,0,0,0,0,0,0,0,0,0,0,0,0,0,0,0,0,0,0,0,0,0,0,0,0,0,0,0,0,0,0,0,0,0,0,0,0,0,0,0,0,0,0$, $0,0,0,0,0,0,0,0,0,0,0,0,0,0,0,0,0,0,0,0,0,0,1,0,0,0,0,0,0,0,0,0,0,0,0,0,0,0,0,0,0,1,0,0,0,0,0,0,1,0,0,0,0,0,1,0$, $0,1,1,0,0,0,0,1,1,1,0,1,0,0,0,0,0,1,1,0,1,0,0,0,0,0,0,0,0,0,0,0,0,0,0,0,0,0,0,0,0,0,0,0,0,0,0,0,0,0,0,0,0,0,0,0$, $0,0,0,0,0,0,0,0,1,0,0,0,0,0,0,0,0,0,0,0,0,0,0,0,0,0,0,0,0,0,0,0,0,1,0,0,0,0,0,0,0,0,0,0,0,0,0,0,0,0,0,0,0,0,0,0$, $0,0,0,0,0,0,0,0,0,0,0,0,0,0,0,0,0,0,0,0,0,0,0,0,0,0,0,0,0,0,0,0,0,0,0,0,0,0,0,0,0,0,0,0,0,0,0,0,0,0,0,0,0,0,0,0$, $0,0,0,0,0,0,0,0,0,0,0,0,0,0,0,0,0,0,0,0,0,0,0,0,0,0,0,0,0,0,0,0,0,0,0,0,0,0,0,1,0,0,0,0,0,0,0,0,0,0,0,0,0,0,0,0$, $0,0,0,0,0,0,0,0,0,0,0,0,0,0,0,0,0,0,0,0,0,0,0,0,0,0,0,0,0,0,0,0,0,0,1,0,0,0,1,0,0,0,0,0,0,0,0,0,0,0,0,0,0,0,0,0$, $0,0,1,0,0,1,0,0,1,0,0,0,0,0,0,0,0,1,0,0,0,0,0,0,1,0,0,0,0,0,0,1,0,1,0,0,0,1,0,0,0,0,0,1,0,0,1,0,0,0,0,0,0,1,0,1$, $0,0,0,0,0,0,0,0,0,0,0,0,0,0,0,0,0,0,0,0,0,1,0,0,0,1,0,0,0,0,0,0,0$

$0,1,0,0,0,0,0,0,0,0,0,0,0,0,0,0,0,0,0,0,0,0,0,0,0,1,0,0,1,0,1,0,0,0,1,1,0,0,0,0,0,0,0,0,0,0,0,0,0,0,0,0,0,0,0,0$, $0,0,0,0,0,0,0,0,0,0,0,0,0,0,0,0,0,0,0,0,0,0,0,0,0,1,0,0,0,0,0,0,0,0,0,0,0,0,1,0,1,0,1,0,0,0,0,1,0,0,0,1,0,1,1,1$, $0,0,1,1,0,0,0,1,0,0,0,0,1,0,0,0,0,0,0,0,0,0,0,0,0,0,1,0,0,0,0,0,0,0,0,0,1,0,0,0,0,0,0,0,0,0,0,0,0,0,0,0,0,0,0,0$, $0,1,0,0,0,0,0,0,0,0,0,0,0,0,0,0,0,0,0,0,0,0,0,0,0,0,0,0,0,0,0,0,0,0,0,0,0,0,0,0,0,0,0,0,0,0,0,0,0,0,0,0,0,0,0,0$, $0,0,1,0,0,0,0,0,0,1,0,0,0,1,0,0,0,0,0,1,1,0,0,0,0,0,1,1,1,1,1,0,0,1,0,0,0,1,0,0,0,0,0,0,0,1,1,0,0,1,0,0,0,0,0,1$, $0,0,0,0,0,0,0,0,0,0,0,0,1,0,0,0,0,0,0,0,0,0,0,0,0,0,0,0,0,0,0,1,0,0,0,0,0,0,0,0,0,0,0,0,0,0,0,0,0,0,0,1,0,0,0,0$, $0,0,0,0,0,0,0,0,0,0,0,0,0,0,0,0,0,0,0,0,0,0,0,0,0,0,0,0,0,0,0,0,0,0,0,0,0,0,0,0,0,0,0,0,0,0,0,0,0,0,0,0,0,0,0,0$, $0,0,0,0,0,0,0,0,0,0,1,0,0,0,0,0,0,1,0,1,0,0,0,0,0,1,0,0,0,1,1,0,0,1,0,0,0,0,0,0,0,1,1,0,0,0,0,0,0,0,0,0,0,0,0,0$, $1,0,0,1,0,1,1,0,0,0,1,0,0,0,0,1,1,0,0,0,0,1,0,0,0,0,0,0,0,0,0,0,0,0,0,0,0,0,0,0,0,0,0,0,0,0,0,0,0,0,0,0,0,0,0,0$, $0,0,0,1,0,0,0,0,0,0,0,0,0,0,0,0,0,0,0,0,0,0,0,0,0,0,0,0,0,1,0,0,0,0,0,0,0,0,0,0,0,0,0,0,0,0,0,0,0,0,0,0,0,0,0,0$, $1,0,0,0,0,0,0,0,0,0,0,0,0,0,0,0,0,0,0,0,0,0,0,0,1,0,0,0,0,0,0,0,0,0,0,0,0,0,0,0,0,0,0,0,0,0,0,0,0,0,0,0,0,0,0,0$, $0,0,0,0,0,1,0,0,0,0,0,0,0,0,0,0,0,0,0,0,0,0,0,0,0,0,0,0,0,0,0,0,0,0,0,0,0,0,0,0,0,0,0,0,0,0,0,1,0,0,0,0,0,0,0,0$, $0,0,1,0,0,0,0,0,0,0,0,0,0,0,0,0,0,0,0,0,0,0,0,0,0,0,0,0,0,0,0,0,0,0,0,0,0,0,0,0,0,0,1,0,0,0,0,0,0,0,0,0,0,0,0,0$, $0,0,0,0,0,0,0,0,0,0,0,0,0,0,0,0,0,0,0,0,0,0,0,0,0,0,0,0,0,0,1,0,0,0,0,0,0,0,0,0,0,1,1,0,1,0,0,1,1,0,0,1,0,0,0,0$, $1,1,1,0,0,0,0,0,0,0,0,0,0,0,0,0,0,0,0,0,0,0,0,0,0,0,0,1,0,0,0,0,0$

$0,0,1,0,0,0,0,0,0,0,0,0,0,1,0,0,1,0,0,0,0,0,0,0,0,0,0,1,0,1,0,0,0,0,0,0,0,0,0,0,1,0,0,0,0,1,0,0,0,0,0,0,0,0,0,0$, $0,0,0,0,0,0,0,0,0,0,0,0,1,0,0,0,0,0,0,0,0,0,0,0,0,0,0,0,0,0,0,0,0,0,0,0,1,0,0,1,0,1,0,0,0,1,0,0,0,0,0,0,0,0,0,0$, 
1469

1470

1471

1472

1473

1474

1475

1476

1477

1478

1479

1480

1481

1482

1483

1484

1485

1486

1487

1488

1489

1490

1491

1492

1493

1494

1495

1496

1497

1498

1499

1500

1501

1502

1503

1504

1505

1506

1507

1508

1509

1510

1511

1512

1513

1514

1515

1516

1517

1518
$0,1,0,0,0,0,0,0,0,0,0,0,0,0,0,0,0,0,0,0,0,0,0,0,0,0,0,0,0,0,0,0,0,0,0,0,0,0,0,0,0,0,0,0,0,0,0,0,0,0,0,0,0,0,0,0$, $0,0,0,0,0,1,0,0,0,0,0,0,0,0,0,0,0,0,1,0,0,0,0,0,0,0,0,0,0,0,0,0,0,0,0,0,1,0,0,0,0,0,0,0,0,0,0,0,0,1,1,0,0,1,0,1$, $0,0,0,1,0,0,0,0,0,0,1,0,0,0,0,0,0,0,0,0,0,1,0,0,0,0,0,0,0,0,0,0,1,0,0,0,0,0,0,0,1,0,0,0,1,0,0,1,0,0,0,0,0,0,0,0$, $0,0,1,0,0,0,0,0,0,0,0,0,0,1,0,0,0,0,0,0,1,0,0,0,0,0,0,1,0,0,0,0,0,0,0,0,0,0,1,1,0,0,0,0,0,0,0,0,0,0,0,0,0,0,0,0$, $0,0,0,0,0,0,0,0,0,0,0,0,0,0,0,0,0,0,0,0,0,0,0,0,0,0,0,0,0,0,0,0,0,0,0,0,0,0,0,0,0,0,1,0,0,0,0,0,0,0,1,0,0,1,0,0$, $0,0,0,0,0,0,0,0,1,0,0,0,1,0,1,1,0,0,0,0,0,0,0,1,1,0,1,0,0,0,0,0,0,0,1,1,0,0,0,1,1,0,0,0,0,0,0,0,0,0,0,0,0,0,0,0$, $0,0,0,0,1,0,0,0,0,0,0,0,1,0,0,0,0,0,0,0,0,0,0,0,0,0,0,0,0,0,0,0,0,0,0,0,0,0,0,0,0,0,0,0,0,0,0,0,0,0,0,0,0,0,0,0$, $0,0,0,0,0,0,0,1,0,0,0,0,0,0,0,0,0,0,0,0,0,1,0,0,0,0,0,0,0,0,0,0,0,0,0,0,0,0,0,0,0,0,0,0,0,0,0,0,0,1,0,0,0,0,0,0$, $0,0,0,0,0,0,0,0,0,0,0,0,0,0,0,0,0,0,0,0,0,0,0,0,0,0,0,0,0,0,0,0,0,0,0,0,0,0,0,0,0,0,0,0,0,0,0,0,0,0,0,0,0,0,0,0$, $0,0,0,0,0,0,0,0,0,0,0,0,0,0,0,0,0,0,0,0,0,0,0,0,0,0,0,0,0,0,0,0,0,0,0,0,0,1,0,0,0,0,0,0,0,0,0,0,0,0,0,0,0,0,0,0$, $0,0,0,0,0,0,0,0,0,0,0,0,0,0,0,0,0,0,0,0,0,0,0,0,0,0,0,0,0,0,0,0,0,1,0,0,0,0,0,0,0,0,0,0,0,0,1,0,0,0,0,0,0,0,0,0$, $0,1,0,0,0,0,1,0,0,1,1,0,0,0,0,1,0,0,0,1,0,0,1,1,0,0,1,0,0,0,0,0,0,0,0,1,0,0,0,0,0,0,0,0,0,0,0,0,0,0,0,0,1,0,1,0$, $0,0,0,1,1,0,0,0,0,0,0,0,0,0,0,0,0,0,0,0,0,0,0,0,0,0,0,0,0,0,0,0,0$

$0,0,0,1,0,1,1,0,0,0,1,1,0,1,0,0,1,0,0,0,0,0,1,0,0,0,1,1,0,1,0,1,0,1,0,0,0,0,1,0,1,0,0,0,0,1,1,1,0,0,0,0,0,0,0,1$, $1,0,0,0,0,0,0,1,0,1,0,0,0,0,1,0,0,0,0,0,0,0,0,0,0,0,0,0,1,1,0,0,1,0,0,0,1,0,0,0,0,1,1,0,0,0,1,0,0,0,0,0,0,0,0,0$, $0,0,0,0,0,0,0,0,0,0,1,1,0,0,0,0,0,0,0,0,0,1,0,0,0,0,1,0,0,0,0,0,0,0,0,1,0,0,0,1,0,0,0,0,0,1,1,0,0,0,0,0,0,0,1,0$, $1,1,0,0,0,1,0,0,1,0,1,0,0,0,0,1,0,0,0,0,0,0,0,0,1,1,0,1,1,0,0,0,0,0,0,0,1,0,1,0,1,0,0,0,0,0,0,0,0,0,0,0,1,1,0,1$, $0,0,0,1,0,0,0,0,0,1,0,0,0,0,1,1,1,0,0,0,0,1,0,0,0,0,0,0,0,0,0,1,0,0,0,0,1,0,0,0,0,0,0,0,1,0,0,0,0,0,0,0,0,0,0,0$, $0,0,0,0,0,0,0,1,0,1,0,0,0,0,0,0,0,1,0,0,0,1,0,0,0,0,0,0,0,0,0,0,0,0,0,0,0,0,0,0,1,1,0,0,0,0,0,0,0,0,1,0,0,0,0,0$, $0,0,1,1,0,0,0,0,0,0,0,0,0,1,0,0,0,0,0,0,0,0,0,0,0,0,0,0,0,1,0,0,1,0,0,0,0,0,0,0,0,0,0,0,1,0,0,0,0,0,0,0,0,1,1,0$, $0,1,0,1,0,0,0,1,0,0,0,0,0,0,1,0,0,0,0,0,0,1,0,0,0,0,0,0,1,0,0,1,0,1,1,1,0,0,0,0,0,0,0,0,0,0,0,0,0,1,0,0,0,0,0,0$, $0,0,0,0,0,0,0,0,0,0,0,0,0,0,1,0,0,0,0,0,0,0,0,0,0,0,0,0,0,0,0,0,0,0,0,0,0,0,0,0,0,0,0,0,0,0,0,0,0,0,0,0,1,0,1,0$, $0,0,0,1,0,1,1,0,1,0,0,0,0,0,1,0,0,0,0,0,0,1,1,0,0,0,0,0,0,0,0,0,0,0,0,0,0,0,0,0,1,1,0,0,1,0,0,0,1,1,0,0,0,0,1,0$, $0,0,0,0,0,0,0,0,0,0,0,0,0,0,0,0,0,0,0,0,0,0,0,0,0,0,0,0,0,0,1,0,0,1,0,0,0,0,0,0,0,0,0,0,0,0,0,1,0,0,0,1,0,0,0,0$, $0,0,1,0,1,0,0,0,0,0,1,1,0,0,0,1,0,0,0,0,0,0,1,1,0,0,0,0,0,0,0,0,0,0,0,0,1,1,0,0,1,0,0,0,0,0,0,1,0,0,0,0,0,0,0,0$, $0,0,0,0,0,0,0,1,0,0,0,0,0,0,0,0,0,0,0,0,0,0,0,0,0,0,0,0,0,0,0,0,0,1,1,0,0,0,1,0,1,0,0,0,0,1,1,0,1,1,0,0,1,0,0,0$, $0,0,1,0,1,0,1,1,1,0,1,0,0,0,0,1,0,0,1,0,0,0,0,0,0,0,1,0,0,1,0,1,0,0,1,0,1,0,0,1,0,0,1,0,1,1,0,0,0,1,0,0,0,0,0,0$, $0,0,0,1,1,0,0,0,0,0,0,0,0,0,0,0,0,0,1,1,0,0,0,0,0,0,0,0,0,1,1,0,1$

$0,0,1,1,1,0,1,1,1,1,1,1,1,0,1,1,1,1,1,1,1,1,1,1,1,1,1,1,0,1,1,1,0,1,0,0,0,0,1,1,0,1,1,1,1,1,1,1,1,1,1,1,1,1,1,1$, $1,1,1,1,1,1,1,1,1,0,1,1,0,1,1,1,1,1,1,1,1,1,1,1,1,1,1,1,0,0,1,1,1,1,0,0,0,1,1,1,1,0,1,1,1,1,1,0,0,0,0,0,0,0,0,0$, $0,0,0,0,0,0,0,0,1,1,0,1,0,1,1,1,1,1,1,1,1,0,1,1,1,1,0,1,1,1,1,1,1,1,1,1,0,1,1,1,1,1,1,1,1,0,0,1,1,1,1,1,1,1,0,1$, $1,1,1,1,1,1,1,1,1,1,0,1,0,1,1,0,1,1,1,1,1,1,1,1,1,1,1,1,0,1,1,1,1,1,1,1,0,1,1,1,1,1,1,1,1,1,1,1,1,0,0,1,0,0,1,1$, $1,0,1,1,1,0,1,0,0,0,1,0,0,1,1,1,1,1,1,1,1,1,0,1,1,1,1,1,1,1,1,0,1,1,1,1,0,1,0,0,1,1,0,0,0,1,0,0,1,0,0,1,0,0,1,0$, $0,1,0,1,1,1,1,1,1,0,1,1,1,1,1,1,1,1,1,1,0,1,1,1,1,1,1,1,1,1,1,1,1,1,1,1,1,1,0,0,0,0,1,1,1,1,1,1,1,1,1,0,1,1,1,1$, $1,1,0,0,1,1,1,1,1,1,1,1,1,1,1,1,1,1,1,1,1,1,1,1,1,1,1,1,1,1,1,1,1,1,1,1,1,1,1,1,1,1,1,1,0,1,1,1,1,1,0,1,1,1,1,1$, $1,1,1,1,1,1,1,1,1,0,1,1,1,1,1,1,0,1,0,1,0,1,1,1,1,1,1,1,1,1,1,1,0,1,1,1,0,0,0,1,1,1,0,1,0,1,1,0,1,1,1,1,0,0,0,0$, $0,0,0,0,0,0,0,0,0,0,0,0,0,0,0,0,0,0,0,1,0,0,1,1,1,1,1,1,1,1,1,1,1,1,1,1,1,1,1,1,1,1,1,1,1,1,1,1,1,1,1,1,0,1,1,1$, $1,1,1,0,1,1,1,1,0,1,1,1,1,1,1,1,1,1,1,1,1,0,1,1,1,1,1,1,1,0,1,1,1,0,1,1,1,1,1,1,1,0,1,1,1,1,1,1,1,0,1,1,1,1,1,1$, $0,1,1,1,1,1,1,1,1,1,1,1,1,1,1,1,1,1,1,1,1,1,1,1,1,1,1,1,1,1,1,1,1,0,1,1,1,1,1,1,1,1,1,1,1,1,1,1,1,1,1,1,1,1,1,1$, $1,1,0,1,1,0,1,1,1,1,1,1,1,1,1,1,1,1,1,1,1,1,1,1,1,1,1,1,1,1,1,1,1,1,1,1,1,1,1,0,1,1,1,1,1,1,1,0,1,1,1,1,1,1,1,1$, $1,1,1,1,1,1,1,0,1,1,1,1,1,1,1,1,1,1,1,1,1,1,1,1,1,1,1,1,1,1,1,1,1,1,0,1,1,1,1,1,0,1,1,0,0,1,1,0,1,1,0,0,1,0,0,1$, $0,0,1,1,0,1,1,1,1,1,1,0,1,0,0,1,0,1,1,1,1,0,1,1,1,0,1,1,1,1,1,0,1,1,1,1,1,1,1,0,1,1,1,1,1,1,1,1,1,1,1,1,1,1,1,1$, $1,1,1,0,0,1,1,1,1,1,1,1,1,1,1,1,1,1,1,1,1,1,0,0,0,0,1,1,1,1,1,1,1$

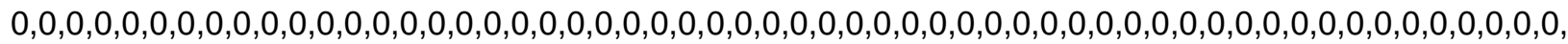
$0,0,0,0,0,0,0,0,0,0,0,0,0,0,0,0,0,0,0,0,0,0,0,0,0,0,0,0,0,0,0,0,0,0,0,0,0,0,0,0,0,0,0,0,0,0,0,0,0,0,0,0,0,0,0,0$, $0,0,0,0,0,0,0,0,0,0,0,0,0,0,0,0,0,0,0,0,0,0,0,0,0,0,0,0,0,0,0,0,0,0,0,0,0,0,0,0,0,0,0,0,0,0,0,0,0,0,0,0,0,0,0,0$, $0,0,0,0,0,0,0,0,0,0,0,0,0,0,0,0,0,0,0,0,0,0,0,0,0,0,0,0,0,0,0,0,0,0,0,0,0,0,0,0,0,0,0,0,0,0,0,0,0,0,0,0,0,0,0,0$, $0,0,0,0,0,0,0,0,0,0,0,0,0,0,0,0,0,0,0,0,0,0,0,0,0,0,0,0,0,0,0,0,0,0,0,0,0,0,0,0,0,0,0,0,0,0,0,0,0,0,0,0,0,0,0,0$, $0,0,0,0,0,0,0,0,0,0,0,0,0,0,0,0,0,0,0,0,0,0,0,0,0,0,0,0,0,0,0,0,0,0,0,0,0,0,0,0,0,0,0,0,0,0,0,0,0,0,0,0,0,0,0,0$, $0,0,0,0,0,0,0,0,0,0,0,0,0,0,0,0,0,0,0,0,0,0,0,0,0,0,0,0,0,0,0,0,0,0,0,0,0,0,0,0,0,0,0,0,0,0,0,0,0,0,0,0,0,0,0,0$, 
1519

1520

1521

1522

1523

1524

1525

1526

1527

1528

1529

1530

1531

1532

1533

1534

1535

1536

1537

1538

1539

1540

1541

1542

1543

1544

1545

1546

1547

1548

1549

1550

1551

1552

1553

1554

1555

1556

1557

1558

1559

1560

1561

1562

1563

1564

1565

1566

1567

1568
$0,0,0,0,0,0,0,0,0,0,0,0,0,0,0,0,0,0,0,0,0,0,0,0,0,0,0,0,0,0,0,0,0,0,0,0,0,0,0,0,0,0,0,0,0,0,0,0,0,0,0,0,0,0,0,0$, $0,0,0,0,0,0,0,0,0,0,0,0,0,0,0,0,0,0,0,0,0,0,0,0,0,0,0,0,0,0,0,0,0,0,0,0,0,0,0,0,0,0,0,0,0,0,0,0,0,0,0,0,0,0,0,0$, $0,0,0,0,0,0,0,0,0,0,0,0,0,0,0,0,0,0,0,0,0,0,0,0,0,0,0,0,0,0,0,0,0,0,0,0,0,0,0,0,0,0,0,0,0,0,0,0,0,0,0,0,0,0,0,0$, $0,0,0,0,0,0,0,0,0,0,0,0,0,0,0,0,0,0,0,0,0,0,0,0,0,0,0,0,0,0,0,0,0,0,0,0,0,0,0,0,0,0,0,0,0,0,0,0,0,0,0,0,0,0,0,0$, $0,0,0,0,0,0,0,0,0,0,0,0,0,0,0,0,0,0,0,0,0,0,0,0,0,0,0,0,0,0,0,0,0,0,0,0,0,0,0,0,0,0,0,0,0,0,0,0,0,0,0,0,0,0,0,0$, $0,0,0,0,0,0,0,0,0,0,0,0,0,0,0,0,0,0,0,0,0,0,0,0,0,0,0,0,0,0,0,0,0,0,0,0,0,0,0,0,0,0,0,0,0,0,1,0,0,0,0,0,0,0,0,0$, $0,0,0,0,0,0,0,0,0,0,0,0,0,0,0,0,0,0,0,0,0,0,0,0,0,0,0,0,0,0,0,0,0,0,0,0,0,0,0,0,0,0,0,0,0,0,0,0,0,0,0,0,0,0,0,0$, $0,0,0,0,0,0,0,0,0,0,0,0,0,0,0,0,0,0,0,0,0,0,0,0,0,0,0,0,0,0,0,0,0$

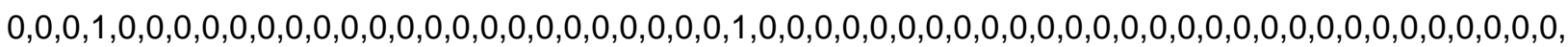
$0,0,0,0,0,0,0,0,0,1,0,0,0,0,1,0,0,0,0,0,0,0,0,0,0,0,0,0,0,0,0,0,0,0,0,0,0,0,0,0,0,0,0,0,0,0,0,0,0,0,0,0,0,0,0,0$, $0,0,0,0,0,0,0,0,0,0,0,0,0,0,0,0,0,0,0,0,0,0,0,0,0,0,0,0,0,0,0,0,0,0,0,0,0,0,0,0,0,0,0,0,0,0,0,0,0,0,0,0,0,0,0,0$, $1,0,0,0,0,0,0,0,0,0,0,0,0,0,0,0,0,0,0,0,0,0,0,0,1,0,0,0,0,0,0,0,0,0,0,0,0,0,0,0,0,0,0,0,0,0,0,0,0,0,0,0,0,0,0,0$, $0,0,0,0,0,0,0,0,0,0,0,0,0,0,0,0,1,0,0,0,0,0,0,0,0,0,0,0,0,0,0,0,0,0,0,1,1,0,0,0,0,0,0,0,0,0,0,0,0,0,0,0,0,0,0,0$, $0,0,0,0,0,0,0,0,0,0,0,0,0,0,0,0,0,0,0,0,0,0,0,0,0,0,0,0,0,0,0,0,0,0,0,0,0,0,0,0,0,0,0,0,0,0,0,0,0,0,1,0,0,0,0,0$, $0,0,0,0,0,0,0,0,0,0,0,0,0,0,0,0,0,0,0,0,0,0,0,0,0,0,0,0,0,0,0,0,1,0,0,0,0,0,0,0,0,0,0,0,1,0,0,0,0,0,0,0,0,0,0,0$, $0,0,0,0,0,0,0,0,0,0,0,0,0,0,0,0,0,0,0,0,0,1,0,0,0,0,0,0,0,0,0,0,0,0,0,0,0,0,0,0,0,0,0,0,0,0,0,0,0,0,0,0,0,0,0,0$, $0,0,0,0,0,0,0,0,0,0,0,0,0,0,0,0,0,0,0,0,0,0,0,0,0,0,0,0,0,0,0,0,0,0,0,0,0,0,0,0,0,0,0,0,0,0,0,0,0,0,0,0,0,0,0,0$, $0,0,0,0,0,0,0,0,0,0,0,0,0,0,0,0,0,0,0,0,0,0,0,0,0,0,0,0,0,0,0,0,0,0,0,0,0,0,0,0,1,1,0,0,0,0,0,0,0,0,0,0,0,0,0,0$, $0,0,0,0,0,0,0,0,0,0,0,0,0,0,0,0,0,0,0,0,0,0,0,0,0,0,0,0,0,0,0,0,0,0,0,0,0,0,0,0,0,0,0,0,0,0,0,0,0,0,0,0,0,0,0,0$, $0,0,0,0,1,0,0,0,0,0,0,0,0,0,0,0,0,0,0,0,0,0,0,0,0,0,0,0,0,0,0,0,0,0,0,0,0,0,0,0,0,0,0,0,0,0,0,0,0,0,0,0,0,0,0,0$, $0,0,0,0,0,0,0,1,0,0,0,0,0,0,0,0,0,0,0,0,0,0,0,0,0,0,0,0,0,0,0,0,0,0,0,0,0,0,0,0,0,0,0,0,0,0,0,0,0,0,0,0,0,0,0,0$, $0,0,0,0,0,0,1,0,0,0,0,0,0,0,0,0,0,0,0,0,0,0,0,0,0,0,0,0,0,0,0,0,0,0,0,0,0,0,0,0,0,0,0,0,0,0,0,0,0,0,0,0,0,0,0,0$, $0,0,0,0,0,0,0,0,0,0,0,0,0,0,0,0,0,0,0,0,0,0,0,0,0,0,0,0,0,0,0,0,0$

$0,0,1,1,0,0,1,0,0,0,1,1,0,1,0,0,1,0,0,0,0,0,0,0,0,1,1,1,0,1,0,1,0,1,0,0,0,0,1,0,1,0,0,0,0,1,1,1,0,0,0,0,0,0,0,1$, $1,0,0,0,0,0,0,1,0,1,0,0,0,0,1,0,0,0,0,0,0,0,0,0,0,0,0,0,1,0,0,0,1,0,0,0,1,0,0,0,0,0,1,0,0,0,1,0,1,0,0,0,0,0,0,0$, $0,0,0,0,0,0,0,0,0,0,1,1,1,0,0,0,0,1,0,0,0,1,0,0,0,0,1,0,0,0,0,0,0,0,0,1,0,0,0,1,0,0,0,0,0,0,0,0,0,0,0,0,0,0,0,0$, $1,1,0,1,0,1,0,0,1,0,0,0,1,0,0,0,0,0,1,0,0,0,0,0,1,1,0,1,1,0,0,0,0,0,0,0,1,0,1,0,1,0,0,0,0,0,0,0,0,1,1,0,1,1,1,1$, $0,0,0,1,1,0,0,0,0,1,0,0,0,1,0,1,1,0,0,1,0,1,0,0,0,0,0,1,1,0,1,1,0,0,0,0,0,0,0,0,1,0,0,0,1,0,0,0,0,0,0,0,0,0,0,0$, $0,0,1,0,0,0,0,1,0,0,0,0,0,1,0,0,1,1,0,0,1,1,0,0,0,0,0,1,0,0,0,0,0,0,0,0,0,0,1,1,1,0,0,0,0,0,0,0,1,0,1,1,0,0,0,0$, $0,0,0,0,0,0,0,0,0,0,0,0,0,1,0,0,0,0,0,0,0,0,0,0,0,0,0,1,0,1,0,0,1,0,0,0,0,0,0,0,0,0,1,0,0,0,0,1,0,0,1,0,0,1,0,0$, $0,1,0,0,0,0,0,0,1,0,0,0,1,1,1,1,0,0,0,0,0,1,0,0,0,0,0,0,1,1,0,1,0,1,1,1,0,0,0,0,0,0,1,1,0,0,0,0,0,1,0,0,0,0,0,0$, $0,0,0,0,0,0,0,0,0,0,0,0,0,0,0,0,0,0,0,0,0,0,0,0,0,0,0,0,0,0,0,0,0,0,0,0,0,0,0,0,0,0,0,0,0,0,0,0,0,0,0,0,1,0,1,0$, $0,0,0,1,0,1,1,1,1,0,0,0,0,0,1,0,0,0,0,0,0,1,1,0,0,0,0,0,0,1,0,0,0,1,0,0,0,0,0,0,0,0,0,0,1,0,0,0,1,1,0,0,0,0,0,0$, $1,0,0,0,0,0,0,0,0,0,0,0,0,0,0,0,0,0,0,0,0,0,0,0,1,0,0,0,0,0,1,0,0,0,0,0,0,0,0,0,0,0,0,0,0,0,0,1,0,0,0,1,0,0,0,0$, $0,0,0,0,1,1,0,0,0,0,1,1,0,0,0,1,0,0,0,0,0,0,1,1,0,0,0,0,0,0,0,0,0,0,0,0,1,1,0,0,0,0,0,0,0,0,0,1,1,0,0,0,0,0,0,0$, $0,0,1,0,0,0,0,0,0,0,0,0,0,0,0,0,0,0,0,0,0,0,0,0,0,0,0,0,0,0,0,0,0,1,1,0,0,0,1,0,1,0,0,0,0,1,1,0,1,0,0,0,1,0,0,0$, $0,0,1,0,1,0,1,1,1,0,0,0,0,0,0,1,0,0,1,0,0,0,0,0,0,0,1,0,0,1,0,0,1,0,1,0,1,0,0,1,0,0,1,0,1,1,0,0,0,1,0,0,0,0,0,0$, $0,0,1,1,1,0,0,0,1,0,0,1,0,1,0,0,0,0,1,0,0,0,0,0,0,0,0,1,0,1,1,0,1$

$1,0,1,0,1,0,0,1,1,1,0,0,1,1,1,1,1,1,1,1,0,1,0,1,1,0,0,0,0,0,1,1,0,1,0,0,0,0,0,1,0,1,1,1,0,1,0,0,1,1,1,1,1,1,1,0$, $0,1,1,1,1,1,1,0,1,0,1,1,0,1,0,1,1,1,1,1,0,1,1,1,1,1,1,1,0,0,1,1,0,1,0,0,0,1,1,1,1,0,0,1,1,1,1,0,0,0,0,0,0,0,0,0$, $1,0,0,0,0,0,0,0,0,1,0,0,0,1,0,1,1,0,1,1,1,0,1,1,1,1,0,1,1,1,1,0,1,1,1,0,0,1,1,0,1,1,1,1,1,0,0,1,1,1,1,1,1,1,0,0$, $0,1,1,1,1,1,1,1,0,1,1,1,1,1,1,1,1,1,0,1,1,1,1,1,0,0,1,0,0,1,1,1,0,1,1,1,1,1,1,1,0,1,1,1,1,1,1,1,1,1,1,1,1,1,1,1$, $1,0,0,0,0,0,1,0,0,0,1,0,0,1,1,1,1,1,1,1,1,1,0,1,1,1,1,1,1,1,1,1,1,1,1,1,1,1,0,0,1,1,0,0,1,1,0,0,0,0,1,0,0,0,0,0$, $0,1,0,1,1,1,1,0,1,0,0,1,1,0,1,1,1,0,1,1,0,0,1,1,1,1,1,0,1,1,1,1,1,1,1,1,1,1,0,0,0,0,1,1,1,1,1,1,1,1,0,0,1,1,1,1$, $1,1,0,0,1,1,1,1,1,1,1,1,1,0,1,1,1,1,1,1,1,1,1,1,1,1,1,0,1,0,1,1,0,1,1,1,1,1,1,1,1,1,0,1,1,1,1,1,1,1,0,1,1,1,1,1$, $1,0,1,1,1,1,1,0,1,0,0,1,0,1,1,1,0,1,0,1,0,1,1,1,1,1,1,1,1,1,1,1,0,1,1,1,0,0,0,1,1,1,0,1,0,1,1,0,1,1,1,1,0,0,0,0$, $0,0,0,0,0,0,0,0,0,0,0,0,0,0,0,0,0,0,0,0,0,0,1,1,1,1,1,1,1,1,1,1,1,1,1,1,1,1,1,1,1,1,1,1,1,1,1,1,1,1,1,1,0,1,0,1$, $1,1,1,1,1,0,0,1,0,1,1,1,1,1,0,1,1,1,1,1,1,1,0,1,1,1,1,1,1,0,1,1,1,1,1,1,1,1,1,1,0,0,1,1,0,1,1,1,0,0,1,1,1,1,0,1$, $0,1,1,1,1,1,1,1,1,1,1,1,1,1,1,1,1,1,1,1,1,1,1,1,1,1,1,1,1,1,0,1,1,0,1,1,1,1,1,1,1,1,1,1,1,1,1,0,1,1,1,0,1,1,1,1$, $1,0,0,1,0,0,1,1,1,1,0,0,1,1,1,0,1,1,1,1,1,1,0,0,1,1,1,1,1,1,1,1,1,1,1,1,0,1,1,0,0,1,1,1,1,1,1,0,1,1,1,1,1,1,1,1$, 
1569

1570

1571

1572

1573

1574

1575

1576

1577

1578

1579

1580

1581

1582

1583

1584

1585

1586

1587

1588

1589

1590

1591

1592

1593

1594

1595

1596

1597

1598

1599

1600

1601

1602

1603

1604

1605

1606

1607

1608

1609

$1610), . \operatorname{Dim}=c(12, X X)))$
$1,1,1,1,1,1,1,0,1,1,1,1,1,1,1,1,1,1,1,1,1,1,1,1,1,1,1,1,1,1,1,1,1,1,1,1,1,1,1,1,1,1,1,0,0,1,0,0,1,0,0,0,0,0,0,1$, $0,1,0,1,0,1,1,1,1,1,0,0,1,0,0,1,0,1,0,1,1,0,1,1,1,0,1,1,1,1,1,1,0,0,1,1,1,1,1,0,1,1,1,1,1,1,1,1,1,1,1,1,1,1,1,1$, $1,1,1,1,1,0,1,1,1,1,1,1,1,0,1,0,1,1,0,1,1,1,0,0,0,0,0,1,1,0,0,1,0$

$0,0,1,1,1,1,1,1,1,1,1,1,1,0,1,1,1,1,1,1,1,1,1,1,1,1,1,1,0,1,1,1,0,1,0,0,0,0,1,1,1,1,1,1,1,1,1,1,1,1,1,1,1,1,1,1$, $1,1,1,1,1,1,1,1,1,1,1,1,0,1,1,1,1,1,1,1,1,1,1,1,1,1,1,1,1,1,1,1,1,1,0,0,1,1,1,1,1,1,1,1,1,1,1,0,0,0,0,0,0,0,0,0$, $0,0,0,0,0,0,0,0,1,1,1,1,1,1,1,1,1,1,1,1,1,1,1,1,1,1,0,1,1,1,1,1,1,1,1,1,1,1,1,1,1,1,1,1,1,1,0,1,1,1,1,1,1,1,1,1$, $1,0,1,1,1,1,1,1,1,1,1,1,1,1,1,1,1,1,1,1,1,1,1,1,1,1,1,1,1,1,1,1,1,1,1,1,1,1,1,1,1,1,1,1,1,1,1,1,1,1,1,1,1,1,1,1$, $1,0,1,1,1,0,1,0,0,1,1,0,0,1,1,1,1,1,1,1,1,1,0,1,1,1,1,1,1,1,1,1,1,1,1,1,1,1,0,0,1,1,0,0,1,1,0,0,0,0,0,0,0,0,0,0$, $0,1,0,1,1,1,1,1,1,1,1,1,1,0,1,1,1,1,1,1,0,1,1,1,1,1,1,0,1,1,1,1,1,1,1,1,1,1,0,0,1,1,1,1,1,1,1,1,1,1,1,1,1,1,1,1$, $1,1,1,1,1,1,1,1,1,1,1,1,1,1,1,1,1,1,1,1,1,1,1,1,1,1,1,1,1,1,1,1,1,1,1,1,1,1,1,1,1,1,0,1,1,1,1,1,1,1,0,1,1,1,1,1$, $1,1,1,1,1,1,1,1,1,0,1,1,1,1,1,1,0,1,0,1,0,1,1,1,1,1,1,1,1,1,1,1,0,1,1,1,0,0,0,1,1,1,1,1,0,1,1,0,1,1,1,1,0,0,0,0$, $0,0,0,0,0,0,0,0,0,0,0,0,0,0,0,0,0,0,0,0,0,0,1,1,1,1,1,1,1,1,1,1,1,1,1,1,1,1,1,1,1,1,1,1,1,1,1,1,1,1,1,1,1,1,1,1$, $1,1,1,1,1,1,1,1,0,1,1,1,1,1,1,1,1,1,1,1,1,0,1,1,1,1,1,1,1,1,1,1,1,1,1,1,1,1,1,1,1,1,1,1,1,1,1,1,1,0,1,1,1,1,1,1$, $1,1,1,1,1,1,1,1,1,1,1,1,1,1,1,1,1,1,1,1,1,1,1,1,1,1,1,1,1,1,1,1,1,1,1,1,1,1,1,1,1,1,1,1,1,1,1,1,1,1,1,1,1,1,1,1$, $1,1,1,1,1,1,1,1,1,1,1,1,1,1,1,1,1,1,1,1,1,1,1,1,1,1,1,1,1,1,1,1,1,1,1,1,1,1,1,1,1,1,1,1,1,1,1,0,1,1,1,1,1,1,1,1$, $1,1,1,1,1,1,1,1,1,1,1,1,1,1,1,1,1,1,1,1,1,1,1,1,1,1,1,1,1,1,1,1,1,1,1,1,1,1,1,1,0,1,1,0,0,1,1,0,1,1,0,0,1,0,0,1$, $0,1,1,1,0,1,1,1,1,1,1,0,1,0,0,1,0,1,1,1,1,0,1,1,1,0,1,1,1,1,1,0,1,1,1,1,1,1,1,1,1,1,1,1,1,1,1,1,1,1,1,1,1,1,1,1$, $1,1,1,1,0,0,1,1,1,1,1,1,1,1,1,1,1,1,1,1,1,1,0,0,0,0,1,1,1,1,1,1,1$

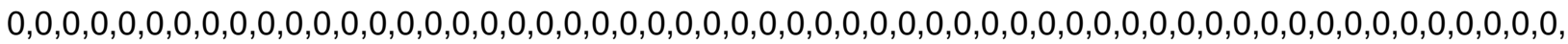
$0,0,0,0,0,0,0,0,0,1,0,0,0,0,0,0,0,0,0,0,0,0,0,0,0,0,0,0,0,0,0,0,0,0,0,0,0,0,0,0,0,0,0,0,0,0,0,0,0,1,0,0,0,0,0,0$, $0,0,0,0,0,0,0,0,0,0,0,0,0,0,0,0,0,0,0,0,0,0,0,0,0,0,0,0,0,0,0,0,0,0,0,0,0,0,0,0,0,0,0,0,0,0,0,0,0,0,0,0,0,0,1,0$, $0,0,0,0,0,0,0,0,0,0,0,0,0,0,0,0,0,0,0,0,0,0,0,0,0,0,0,0,0,0,0,0,0,0,0,0,0,0,0,0,0,0,0,0,0,0,0,0,0,0,0,0,0,0,0,0$, $0,0,0,0,0,0,0,0,0,0,1,0,0,0,0,0,0,0,1,0,0,0,0,0,0,0,0,0,0,0,0,0,0,0,0,0,0,0,0,0,0,0,0,0,0,0,0,0,0,0,0,0,0,0,0,0$, $0,0,0,0,0,0,0,0,0,0,0,0,0,0,0,0,0,0,0,0,0,0,1,0,1,1,0,0,0,0,0,0,0,0,0,0,0,1,0,1,0,0,1,0,0,1,0,0,0,0,0,0,0,0,0,0$, $0,0,0,0,0,0,0,1,0,0,0,0,0,0,0,0,0,0,0,0,0,0,0,0,0,0,0,0,0,0,0,0,0,0,0,0,0,0,0,0,0,0,0,0,0,0,0,0,0,0,0,0,0,0,0,0$, $0,0,0,0,0,0,0,0,0,0,0,0,0,0,0,0,0,0,0,0,0,0,0,1,0,0,0,0,0,1,0,1,0,0,0,0,0,0,0,0,0,0,0,0,0,0,0,0,0,0,0,0,0,0,0,0$, $0,0,0,0,0,0,0,0,0,0,0,0,0,0,0,0,0,0,0,0,0,0,0,0,0,0,0,0,0,0,0,0,0,0,0,0,0,0,0,0,0,0,0,0,0,0,0,0,0,0,0,0,0,0,0,0$, $0,0,0,0,0,0,0,0,0,0,0,0,0,0,0,0,0,0,0,0,0,0,0,0,0,0,0,0,0,0,0,0,0,0,0,0,0,0,0,0,0,0,0,0,0,0,0,0,0,0,0,0,0,0,0,0$, $1,0,0,0,0,0,0,0,0,0,0,0,0,0,0,0,0,0,0,0,0,0,0,0,0,0,0,0,0,0,0,0,0,0,0,0,0,0,0,0,0,0,0,0,0,0,0,0,0,0,0,0,0,0,0,0$, $0,0,0,0,0,0,0,0,0,0,0,0,0,0,0,0,0,0,0,0,0,0,0,0,0,0,0,0,0,0,0,0,0,1,0,0,0,0,0,0,0,0,0,0,0,0,0,0,0,0,0,0,0,0,0,0$, $0,0,0,0,0,0,0,0,0,0,0,0,0,0,0,0,0,0,0,0,0,0,0,0,0,0,0,0,0,0,0,0,0,0,0,0,0,0,0,0,0,0,0,0,0,0,0,0,0,0,0,0,0,0,0,0$, $0,0,0,0,0,0,0,0,0,0,0,0,0,0,0,0,0,0,0,0,0,0,0,0,0,0,0,0,0,0,0,0,0,0,0,0,0,0,0,0,0,0,0,0,0,0,0,0,0,0,0,0,0,0,0,0$, $0,0,0,0,0,0,0,0,0,0,0,0,0,0,0,0,0,0,0,0,0,0,0,0,0,0,0,0,0,0,0,0,0$ 
1612 \# INITIAL VALUES

1613

1614 list $($ PriorS $=c(0.1,0.1,0.1,0.1,0.1,0.1,0.1,0.1,0.1,0.1,0.1,0.1,0.1,0.1,0.1,0.1,0.1,0.1,0.1))$

1615

1616 list(Priors

$1617=\mathrm{c}(0.01,0.01,0.01,0.01,0.01,0.01,0.01,0.01,0.01,0.01,0.01,0.01,0.01,0.01,0.01,0.01,0.01,0.01,0.01))$

1618

1619 list $($ PriorS $=c(0.2,0.2,0.2,0.2,0.2,0.2,0.2,0.2,0.2,0.2,0.2,0.2,0.2,0.2,0.2,0.2,0.2,0.2,0.2))$

1620

1621 list(PriorS

$1622=c(0.05,0.05,0.05,0.05,0.05,0.05,0.05,0.05,0.05,0.05,0.05,0.05,0.05,0.05,0.05,0.05,0.05,0.05,0.05))$

1623

1624 list $($ PriorS $=c(0.3,0.3,0.3,0.3,0.3,0.3,0.3,0.3,0.3,0.3,0.3,0.3,0.3,0.3,0.3,0.3,0.3,0.3,0.3))$

1625

1626

1627

1628

1629

1630

1631

1632

1633

1634

1635

1636

1637

1638

1639

1640

1641

1642

1643

1644

1645

1646

1647

1648

1649

1650

1651

1652

1653

1654

1655

1656

1657

1658 
1659 \# model for SALMONELLA and 12 sources (including water): locations' sub-analysis

1660 \# data set: first all simple foods (o in s1: S)

1661 \# complex foods (o in $\mathrm{c} 1: \mathrm{C}$ )

1662

1663

1664 \# variables:

1665 \# out[i] = outbreak per pathogen

1666 \# s = outbreak observation in simple foods

$1667 \quad \# \mathrm{i}=$ outbreak observation in complex foods

$1668 \# \mathrm{j}=$ source

1669 \# PriorS = prior knowledge we gain from simple food outbreaks for each source

$1670 \quad \# \mathrm{~F}=$ presence or absence of source $\mathrm{j}$ in complex food $\mathrm{f}$, matrix

1671 \#sources:

1672 \#1Eggs2Dairy3GoatMilk4Meat5Poultry6Chicken7Ducks8Turkey9Beef10Pork11Lamb12Mutton13Game14F

1673 ruitsNuts15Vegetables16GrainsBeans17OilsSugar18Seafood19Water

1674

1675 \#1Beef2Pork3Poultry4Meat5Dairy6Eggs7Seafood8FruitsNuts9GrainsBeans10Vegetables11OilSugar12

1676 Water

1677

1678

1679 model

1680\{

1681

1682 \# Proportion of unknowns

1683

1684

1685

1686

1687 propukG <- unknownG * 100/ totaloutbreaksG

1688 propukH $<-$ unknownH * 100/ totaloutbreaksH

1689

1690

1691 \# Variable to estimate attribution of complex foods, separated loop (out of order) 
1692

1693 \#G (General)

1694 for (i in Gc1:Gc2 ) \{

$1695 b[i]<-\operatorname{sum}(a[1: 12, i])$

1696

$1697\}$

1698

1699 \#H (Household)

1700 for (i in Hc1:Hc2) \{

$1701 \quad b[i]<-\operatorname{sum}(a[1: 12, i])$

1702

$1703\}$

1704

1705

1706

1707 for $(\mathrm{j}$ in $1: 12)$ \{

1708

1709 \# PRIOR

1710

1711

1712 sourceS[j] <- sum(S[j,1:totalS $])$

1713

1714

$1715 z[i]<-$ sourceS[j] +1

$1716 y[j]<-$ totalS - sourceS[j] +1

1717 PriorS[j] dbeta(z[j], y[j])

1718

1719

1720

1721

1722

1723 
1724 \# BY LOCATION

1725

1726

$1727 \quad$ \#G

1728

1729 \# SIMPLE FOODS

1730 sourceSG[j] <- sum(F[j,Gs1:Gs2])

1731

1732 \# COMPLEX FOODS

1733 for (i in Gc1:Gc2) \{

1734

$1735 a[j, i]<-$ PriorS[j] * F[j,i]

$1736 c[j, i]<-a[j, i] / b[i]$

1737

$1738\}$

1739

1740

$1741 \quad$ \#H

1742

1743 \# SIMPLE FOODS

1744 sourceSH[j] <- sum(F[j,Hs1:Hs2])

1745

1746 \# COMPLEX FOODS

1747 for (i in Hc1:Hc2) \{

1748

$1749 a[\mathrm{a}, \mathrm{i}]<-$ PriorS[j] * $\mathrm{F}[\mathrm{j}, \mathrm{i}]$

$1750 \quad c[j, i]<-a[j, i] / b[i]$

1751

$1752\}$

1753

1754 
1755

1756

1757

1758 sourceCG[j] <- sum(c[j,Gc1:Gc2 ])

1759 sourceCH[j] <- sum(c[j,Hc1:Hc2])

1760

1761

1762 TotalG[j] <- sourceSG[j] + sourceCG[j]

1763 TotalH[j] <- sourceSH[j] + sourceCH[j]

1764

1765

1766 AttribG[j] <- $($ TotalG[j] * 100) / totaloutbreaksG

1767 AttribH[j] <- $($ TotalH[j] * 100) / totaloutbreaksH

1768

$1769\}$

$1770 \quad\}$

1771

1772

1773 \# DATA

1774

1775

1776 list(

1777

1778

1779 Gs1 = 1 ,

1780 Gs2 $=20$,

$1781 \mathrm{Gc1}=21$,

1782 Gc2 $=243$,

1783

$1784 \mathrm{Hs} 1=313$,

1785 Hs2 $=354$,

$1786 \mathrm{Hc} 1=355$, 
$1787 \mathrm{Hc} 2=695$,

1788

1789

1790 unknownG $=69$,

1791 unknownH = 122 ,

1792

1793

1794 totaloutbreaks $\mathrm{G}=312$,

1795 totaloutbreaksH $=505$,

1796

1797 totalS $=61$,

1798

1799

1800

1801

1802

1803

1804 S=structure $($.Data $=$

$1805 \mathrm{c}(0,1,0,0,0,1,0,1,0,0,0,0,0,0,0,0,0,0,0,0,0,0,1,0,0,0,0,0,0,0,0,0,0,0,0,0,0,0,0,0,0,0,1,0,1,0,0,0,0,0,0,0,0,0,0$, $18060,0,0,0,0,0$

$1807 \quad 0,0,1,0,0,0,0,0,0,0,0,0,0,0,0,1,0,0,1,0,0,0,0,0,1,0,1,0,0,0,0,0,0,1,1,1,0,0,0,0,0,1,0,0,0,0,0,0,1,0,0,0,1,1,0,1$, $18081,1,1,1,1$,

$18091,0,0,1,0,0,0,0,0,1,0,1,1,1,1,0,0,1,0,1,0,1,0,1,0,1,0,1,0,0,1,0,1,0,0,0,0,1,0,1,1,0,0,0,0,0,1,1,0,0,0,1,0,0,1,0$, $1810 \quad 0,0,0,0,0$,

$18110,0,0,0,0,0,1,0,0,0,1,0,0,0,0,0,0,0,0,0,0,0,0,0,0,0,0,0,1,0,0,1,0,0,0,0,0,0,0,0,0,0,0,0,0,0,0,0,0,0,0,0,0,0,0,0$, $18120,0,0,0,0$,

$18130,0,0,0,0,0,0,0,0,0,0,0,0,0,0,0,0,0,0,0,0,0,0,0,0,0,0,0,0,0,0,0,0,0,0,0,0,0,0,0,0,0,0,0,0,1,0,0,0,0,0,0,0,0,0,0$, $18140,0,0,0,0$,

$18150,0,0,0,0,0,0,0,0,0,0,0,0,0,0,0,1,0,0,0,0,0,0,0,0,0,0,0,0,1,0,0,0,0,0,0,1,0,0,0,0,0,0,1,0,0,0,0,0,0,1,0,0,0,0,0$, $18160,0,0,0,0$,

$18170,0,0,0,0,0,0,0,0,0,0,0,0,0,0,0,0,0,0,0,0,0,0,0,0,0,0,0,0,0,0,0,0,0,0,0,0,0,0,0,0,0,0,0,0,0,0,0,0,0,0,0,0,0,0,0$, $18180,0,0,0,0$,

$18190,0,0,0,0,0,0,0,0,0,0,0,0,0,0,0,0,0,0,0,0,0,0,0,0,0,0,0,0,0,0,0,0,0,0,0,0,0,0,0,0,0,0,0,0,0,0,0,0,0,0,0,0,0,0,0$, $1820 \quad 0,0,0,0,0$,

$18210,0,0,0,0,0,0,0,0,0,0,0,0,0,0,0,0,0,0,0,1,0,0,0,0,0,0,0,0,0,0,0,0,0,0,0,0,0,0,0,0,0,0,0,0,0,0,0,0,0,0,0,0,0,0,0$, $18220,0,0,0,0$ 
1823

1824

1825

1826

1827

1828

1829

1830

1831

1832

1833

1834

1835

1836

1837

1838

1839

1840

1841

1842

1843

1844

1845

1846

1847

1848

1849

1850

1851

1852

1853

1854

1855

1856

1857

1858

1859

1860

1861

1862

1863

1864

1865

1866

1867
$0,0,0,0,1,0,0,0,1,0,0,0,0,0,0,0,0,0,0,0,0,0,0,0,0,0,0,0,0,0,0,0,0,0,0,0,0,0,0,0,0,0,0,0,0,0,0,0,0,1,0,0,0,0,0,0$, $0,0,0,0,0$

$0,0,0,0,0,0,0,0,0,0,0,0,0,0,0,0,0,0,0,0,0,0,0,0,0,0,0,0,0,0,0,0,0,0,0,0,0,0,0,0,0,0,0,0,0,0,0,0,0,0,0,0,0,0,0,0$, $0,0,0,0,0$

$0,0,0,0,0,0,0,0,0,0,0,0,0,0,0,0,0,0,0,0,0,0,0,0,0,0,0,0,0,0,0,0,0,0,0,0,0,0,1,0,0,0,0,0,0,0,0,0,0,0,0,0,0,0,0,0$, $0,0,0,0,0$

)$, . \operatorname{Dim}=c(12,61))$

$\mathrm{F}=$ structure $($. Data $=$

$c($

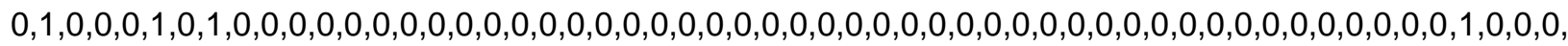
$0,0,0,0,0,0,0,0,0,0,0,0,0,0,0,0,0,0,0,0,0,0,0,0,0,0,0,1,0,0,0,0,0,0,0,0,0,0,0,0,0,0,0,0,0,0,0,0,0,0,0,0,0,0,0,0$, $0,0,0,0,0,1,0,0,0,0,0,0,1,0,0,0,1,0,0,0,0,0,0,0,0,0,0,0,0,0,0,0,0,0,0,0,1,0,0,0,0,0,0,0,0,0,0,0,0,0,0,1,0,0,0,0$, $0,0,0,0,0,0,0,0,0,0,1,0,0,0,0,0,0,0,0,0,0,0,0,0,0,0,0,0,0,0,0,0,0,0,0,0,0,0,0,0,0,0,0,0,0,0,0,0,0,0,0,0,0,0,0,0$, $0,0,0,0,0,0,0,0,0,0,0,0,0,0,0,0,0,0,0,0,0,0,0,0,1,0,0,0,0,0,0,0,0,0,0,1,0,1,0,1,0,0,0,0,0,0,0,0,0,1,0,0,0,0,0,0$, $0,0,1,0,0,0,0,0,0,0,0,0,0,0,0,0,0,0,0,0,1,1,0,0,1,0,0,0,0,1,1,0,0,0,1,0,0,0,0,0,0,0,0,0,0,0,0,0,0,0,0,0,0,0,1,0$, $1,0,0,0,0,0,0,0,0,0,0,0,0,0,0,0,0,0,0,0,0,0,0,0,0,0,0,0,0,0,0,0,0,0,0,0,0,0,0,0,0,0,0,0,0,0,0,0,0,0,0,0,0,0,1,0$, $0,0,0,0,0,0,1,0,0,0,0,0,0,0,0,0,0,0,0,0,0,0,0,0,0,0,0,0,0,0,0,0,0,0,0,0,0,0,0,0,0,0,1,0,0,0,0,0,0,0,0,0,0,0,0,0$, $0,0,0,0,0,0,0,0,0,0,0,0,0,0,0,0,0,0,0,0,0,0,0,0,0,0,0,0,0,0,0,0,0,0,0,0,0,0,0,0,0,0,0,0,0,0,0,0,0,0,0,0,0,0,0,0$, $0,0,0,0,0,0,0,0,0,0,0,0,0,0,0,0,0,0,0,0,0,0,0,0,0,0,0,0,0,0,0,0,0,0,0,0,0,0,0,0,0,0,0,0,0,0,0,0,0,0,0,0,0,0,0,0$, $0,0,0,0,0,0,0,0,0,0,0,0,0,0,0,0,0,0,0,0,0,0,0,0,0,0,0,0,0,0,0,0,0,0,0,0,0,0,0,0,0,0,0,0,0,0,0,0,0,0,0,0,0,0,0,0$, $0,0,0,0,0,0,0,0,0,0,0,0,0,0,0,0,0,0,0,0,0,0,0,0,0,0,0,0,0,0,0,0,0,0,0,0,0,0,0,0,0,0,0,0,0,0,0,0,0,0,0,0,0,0,0,0$, $0,0,0,0,0,0,0,0,0,0,0,0,0,0,0,0,0,0,0,0,1,0,0,0,0,0,0,0,0,0,0,0,0,0,1,0,0,0,0,0,0,0,0,1,1,0,0,0,1,0,1,0,0,0,0,0$, $0,1,0,0,0,0,0,0,0,0,0,0,0,1,0,0,0,1,0,0,0,0,0,0,1,0,0,1,0,0,0,0,0,0,0,0,0,0,0,0,0,1,0,0,0,0,0,0,0,0,0,1,1,0,0,0$,

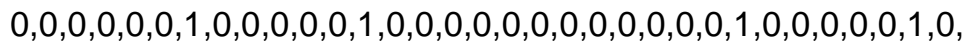

$0,0,1,0,0,0,0,0,0,0,0,0,0,0,0,1,0,0,1,0,0,0,0,0,0,0,0,0,0,0,0,0,0,0,0,0,0,0,0,0,0,0,0,0,0,0,0,0,0,0,0,0,0,0,0,0$, $0,0,0,0,0,0,0,0,0,0,0,0,0,1,0,0,0,0,0,0,0,0,0,0,0,0,0,0,0,0,0,0,0,0,0,0,0,0,0,0,0,0,0,0,0,0,0,0,0,0,0,0,0,0,0,0$, $0,0,0,0,0,0,0,0,0,0,0,0,0,0,0,0,0,0,0,0,0,0,0,0,0,0,0,0,0,0,0,0,0,0,0,1,0,0,0,0,0,0,0,0,1,0,0,0,0,0,0,0,0,0,0,0$, $0,0,0,0,0,0,0,0,0,0,0,0,0,0,1,0,0,0,0,0,0,0,0,0,0,0,0,0,0,0,0,0,0,0,0,1,0,0,0,0,0,0,0,0,0,1,0,0,0,0,0,0,0,0,0,0$, $0,0,0,0,0,0,0,0,0,0,0,0,0,0,0,0,0,0,0,0,1,0,0,0,0,0,0,0,0,0,0,0,0,0,0,0,0,0,0,0,0,1,0,0,1,0,0,0,0,0,0,0,0,0,0,0$, $0,0,0,0,0,0,0,0,0,0,0,0,0,0,0,0,0,0,0,0,0,0,0,0,0,0,1,1,1,0,0,0,0,0,0,0,1,0,1,0,0,0,0,0,0,1,1,1,0,0,0,0,0,1,0,0$, $0,0,0,0,1,0,0,0,1,1,0,1,1,1,1,1,1,0,0,0,0,0,0,0,0,0,0,0,0,0,0,0,0,0,0,0,0,0,0,0,0,0,0,0,0,0,0,0,0,0,0,0,0,0,0,0$, $0,0,0,0,0,0,0,0,0,0,0,0,0,0,0,0,0,0,0,0,0,0,0,0,0,0,0,0,0,0,0,0,0,0,0,0,0,0,0,0,0,0,0,0,0,0,0,0,0,0,0,0,0,0,0,0$, $0,0,0,0,0,0,0,0,0,0,0,0,0,0,0,0,0,0,0,0,0,0,0,0,1,0,0,0,0,0,0,0,0,1,0,0,0,0,0,0,0,0,0,0,0,0,0,0,0,0,0,0,0,0,0,0$, $0,0,0,0,0,0,0,0,0,0,0,0,0,0,0,0,0,0,0,0,0,0,0,0,0,0,0,0,0,0,0,0,0,0,0,0,0,0,0,0,0,0,0,0,0,0,0,0,0,0,0,0,0,0,0,0$, $0,0,0,0,0,0,0,0,0,0,0,0,0,0,0,0,0,0,0,0,0,0,0,0,0,0,0,0,0,0,0,0,0,0,0,0,0,0,0,0,0,0,0,0,0,0,0,0,0,0,0,0,0,0,0,0$, $0,0,0,0,0,0,0,0,0,0,0,0,0,0,0,0,0,0,0,0,0,0,0,0,0,0,0,0,0,0,0,0,0,0,0,0,0,0,0,0,0,0,0,0,0,0,0,0,0,0,0,0,0,0,0,0$, $0,0,0,0,0,0,0,0,0,0,0,0,0,0,1,0,0,0,0,0,0,0,0,0,0,0,0,0,0,0,1,0,0,0,0,0,0,0,0,0,1,0,0,0,0,0,0,0,0,1,0,0,0,0,0,0$, $0,0,1,0,0,0,0,0,0,0,0,0,0,1,0,0,0,0,0,0,0,0,0,0,0,0,1,0,0,1,0,1,0,0,0,0,0,0,0,1,0,0,0,0,0,1,0,0,0,0,0,0,0,0,0,1$, $0,0,1,0,0,0,0,0,1,0,0,0,0,0,0,0,1,1,1,0,0,0,0,0,0,0,0,0,0,0,0,0,0$,

$1,0,0,1,0,0,0,0,0,1,0,1,1,1,1,0,0,1,0,1,0,0,0,0,0,0,0,0,0,1,0,0,0,0,0,0,0,0,0,0,0,0,0,0,0,0,0,0,0,0,0,0,0,0,0,0$, $0,0,0,0,0,0,0,0,1,0,0,0,0,0,0,0,0,1,0,0,0,0,0,0,0,0,0,0,0,0,0,0,0,0,0,0,0,0,0,0,0,1,0,0,0,0,0,0,0,0,0,0,0,0,0,0$, $0,0,0,0,0,0,0,0,0,0,0,0,0,0,0,0,0,0,0,0,0,0,0,0,0,0,0,0,0,0,0,0,0,0,0,0,0,0,0,0,0,0,0,0,0,0,0,0,0,0,0,0,0,0,0,0$, $0,0,0,0,0,1,0,0,0,0,0,0,0,0,0,0,0,0,0,0,0,0,0,0,0,0,0,0,0,0,0,0,0,1,0,0,0,0,0,0,0,0,0,0,0,0,0,0,0,0,0,0,0,0,0,0$, 
1868

1869

1870

1871

1872

1873

1874

1875

1876

1877

1878

1879

1880

1881

1882

1883

1884

1885

1886

1887

1888

1889

1890

1891

1892

1893

1894

1895

1896

1897

1898

1899

1900

1901

1902

1903

1904

1905

1906

1907

1908

1909

1910

1911

1912

1913

1914

1915

1916

1917
$0,0,0,0,0,0,0,0,0,0,1,0,0,0,0,0,0,0,0,1,0,1,0,1,0,0,1,0,1,1,1,1,1,1,0,0,1,0,0,0,0,0,0,0,0,0,0,0,0,0,0,0,0,0,0,0$, $0,1,0,0,0,0,1,0,0,0,1,0,0,0,0,0,0,0,0,0,0,0,0,0,0,1,0,0,0,0,0,0,0,1,0,1,0,1,0,1,0,0,1,0,1,0,0,0,0,1,0,1,1,0,0,0$, $0,0,1,1,0,0,0,1,0,0,1,0,0,0,0,0,0,0,0,0,0,0,0,0,0,0,0,0,0,0,0,0,0,0,0,0,0,0,0,0,0,0,0,0,0,0,0,0,0,0,0,0,0,0,0,0$, $1,0,0,0,0,0,0,0,0,0,0,0,0,0,1,0,0,0,0,0,0,0,0,0,0,0,0,1,0,0,0,0,0,0,0,0,0,0,0,0,0,0,0,0,0,0,0,0,1,0,0,0,0,0,0,0$, $0,0,0,0,0,0,0,0,0,0,0,0,0,0,0,0,0,0,0,0,0,0,0,0,0,0,0,0,0,0,0,0,0,0,0,0,0,0,0,0,0,0,0,0,0,0,0,0,0,0,0,0,0,0,0,0$, $0,0,0,0,0,0,0,0,0,0,0,0,0,0,0,0,0,0,0,0,0,0,0,0,0,0,0,0,0,0,0,0,1,0,0,0,0,0,0,0,0,0,0,0,0,0,0,0,0,0,0,0,1,0,0,0$, $0,0,0,0,0,0,0,0,0,0,0,0,0,1,0,0,0,0,0,0,0,0,0,0,0,0,0,0,0,0,0,0,0,0,0,0,0,0,0,0,0,0,0,0,0,0,0,0,0,0,0,0,0,0,0,0$, $0,0,0,0,0,0,0,0,1,0,0,0,0,0,1,0,0,0,0,0,0,0,0,0,0,0,0,0,0,0,0,0,0,0,0,0,0,0,0,0,1,0,0,0,0,0,0,0,0,0,0,0,0,0,0,0$, $0,0,0,0,0,0,0,0,0,0,0,0,0,1,0,0,0,0,0,0,0,0,0,0,0,0,0,0,1,0,0,0,0,0,0,0,0,0,0,0,0,0,0,0,0,1,0,0,0,0,0,1,0,0,1,0$, $0,0,0,0,1,0,1,0,1,0,0,0,0,1,1,0,0,0,0,0,0,0,0,0,0,0,0,0,0,0,0,0,0,0,0,0,0,0,0,0,0,0,0,0,0,0,0,0,0,0,0,0,0,1,1,0$, $1,0,0,0,1,1,0,0,0,1,1,0,0,1,0,0,0,0,0,1,1,0,0,0,0,0,1,0,0,1,1,0,0$,

$0,0,0,0,0,0,1,0,0,0,1,0,0,0,0,0,0,0,0,0,0,0,0,0,0,1,0,0,0,0,0,1,0,0,0,1,0,0,0,0,0,0,0,0,0,0,0,0,0,0,0,0,0,0,0,0$, $0,0,0,0,0,0,0,0,0,0,1,0,0,0,0,0,0,0,0,0,0,0,0,0,0,0,0,0,0,0,0,0,0,0,0,0,0,0,0,0,0,0,0,0,0,0,0,0,0,0,0,0,0,1,0,0$, $0,1,0,0,1,0,0,0,0,0,0,0,0,0,0,0,0,1,0,0,0,0,0,0,0,0,0,1,0,0,0,1,1,1,0,0,0,1,0,0,0,0,0,0,0,0,0,0,0,0,0,0,0,1,0,0$, $0,1,0,0,0,0,0,0,1,1,0,0,0,0,0,0,0,0,0,0,0,0,0,0,0,0,0,0,0,0,0,0,0,0,1,0,0,0,0,0,0,0,0,0,0,0,0,0,0,0,0,0,0,0,0,0$, $0,0,0,0,0,0,0,0,0,0,0,0,0,0,0,0,0,0,0,0,0,0,1,0,0,0,0,0,0,0,0,0,0,0,0,0,0,0,0,0,1,0,0,0,0,0,0,0,0,0,0,1,0,1,1,1$, $0,0,0,0,1,1,0,1,1,0,0,0,1,1,0,1,0,0,1,0,0,0,1,0,0,0,0,0,0,0,0,0,0,0,0,0,0,0,0,0,1,0,0,1,0,0,0,0,0,0,0,0,0,0,0,0$, $0,0,0,0,0,0,0,0,0,0,0,0,0,0,0,0,0,1,0,0,0,0,0,0,1,0,0,0,0,0,0,0,0,0,0,0,0,0,0,0,0,0,0,0,0,0,1,0,0,0,0,0,0,0,0,0$, $0,0,0,0,0,0,0,0,0,0,0,0,0,0,0,0,0,0,0,0,0,1,0,0,0,0,0,0,0,0,0,0,0,0,0,0,0,1,0,0,0,0,0,0,0,0,0,0,0,0,0,0,0,0,0,0$, $0,0,0,0,0,0,0,0,0,0,0,0,0,0,0,0,0,0,0,0,0,0,1,0,0,0,0,0,0,0,0,0,0,0,0,0,0,0,0,0,0,0,0,0,0,0,0,0,0,0,0,0,0,0,0,0$, $0,0,0,0,0,0,0,0,0,0,0,0,0,0,0,0,0,0,0,0,0,0,0,0,0,1,0,0,0,0,0,0,0,0,0,0,0,0,0,0,0,0,0,0,0,0,0,1,0,0,0,0,0,0,0,0$, $0,0,0,0,0,0,0,0,0,0,0,0,0,0,0,0,0,0,0,0,0,0,0,0,0,0,0,0,0,0,0,0,0,0,0,0,0,0,0,0,0,0,0,0,0,0,0,0,0,0,0,0,0,0,0,0$, $0,0,0,0,0,0,0,0,0,0,0,0,0,0,0,0,0,0,0,0,0,0,0,0,0,0,0,0,0,0,0,0,0,0,0,0,0,0,0,0,0,0,0,0,0,0,0,0,0,0,0,0,0,0,0,0$, $0,0,0,0,0,0,1,0,0,0,0,0,0,0,0,0,1,0,0,0,0,0,0,1,0,0,0,1,0,1,0,1,1,0,0,0,0,1,0,0,0,0,0,0,0,0,1,0,0,0,0,0,1,0,0,0$, $0,0,0,1,0,0,0,0,0,0,0,1,1,0,0,0,0,0,0,0,0,0,0,0,0,0,0,0,0,0,1,0,1,1,0,0,0,1,0,0,1,0,0,1,1,0,0,0,0,0,1,0,0,0,0,0$,

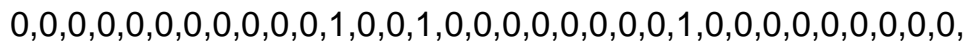

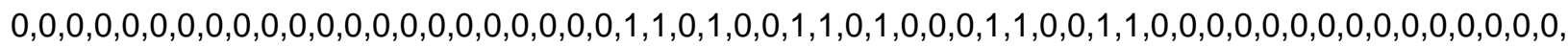
$0,0,1,0,0,1,0,0,0,0,1,0,0,0,0,0,0,1,0,0,0,0,0,0,0,0,1,1,0,0,0,0,0,1,0,0,0,1,0,0,0,1,0,0,0,0,0,1,0,0,0,0,0,0,0,1$, $0,1,0,0,0,1,0,0,0,0,1,1,1,0,1,0,0,0,0,0,0,0,0,0,0,1,0,1,1,0,0,1,0,0,0,1,1,1,1,0,0,0,0,0,1,1,1,0,0,0,0,1,0,0,0,0$, $0,0,0,0,0,0,0,0,0,0,1,1,0,0,0,0,0,0,0,0,1,0,0,0,0,0,0,0,0,0,0,0,1,1,0,1,0,0,1,0,0,0,0,0,0,0,1,0,0,1,0,0,0,0,0,0$, $0,0,0,1,1,0,0,0,1,0,0,0,0,0,0,0,0,0,0,0,0,0,1,1,0,0,0,0,1,0,0,0,0,0,1,1,0,0,0,0,0,0,0,1,0,0,1,0,0,0,0,1,0,1,1,0$, $0,0,0,0,1,0,0,0,0,1,0,1,1,1,0,1,1,1,0,1,0,1,1,0,0,0,1,0,0,0,1,0,0,0,0,0,0,0,0,0,0,0,0,0,0,0,0,0,0,0,0,0,0,0,0,0$, $0,1,0,0,0,0,0,0,0,0,0,0,0,0,0,0,0,0,1,0,1,1,0,0,1,0,0,0,1,0,1,0,0,0,0,0,1,0,0,0,0,0,0,1,0,0,0,0,1,0,0,0,0,0,1,1$, $0,0,0,0,0,0,1,0,0,0,0,0,0,1,0,0,1,0,0,0,0,0,0,0,1,0,0,0,0,1,0,1,0,0,0,0,0,0,0,0,0,0,0,0,0,0,0,1,0,0,0,0,1,1,0,0$, $0,0,0,0,0,0,1,0,0,0,0,0,0,0,0,0,0,0,0,0,0,1,1,0,0,0,0,0,0,0,0,1,0,0,0,0,1,0,1,0,0,0,0,0,0,0,0,0,0,0,0,0,0,0,0,0$, $0,0,0,0,0,0,0,0,0,0,1,0,0,0,0,0,0,1,1,0,0,0,0,0,0,1,1,0,0,0,0,0,0,0,0,0,0,0,0,0,1,1,0,0,0,0,1,1,0,0,0,0,0,0,0,0$, $0,0,0,0,0,0,0,0,0,0,0,0,0,0,0,0,0,0,0,0,0,0,0,0,0,0,0,0,0,1,0,0,0,0,0,0,0,0,0,1,1,0,0,0,1,1,0,1,0,0,0,0,0,1,1,0$, $0,0,0,0,0,0,0,0,0,0,0,0,0,0,0,0,0,0,0,1,1,0,1,0,0,0,0,0,0,0,0,0,0,0,0,0,0,0,0,1,0,0,0,0,0,0,0,0,0,0,0,0,0,0,0,0$, $0,0,0,0,0,1,1,0,0,0,1,0,1,0,0,0,1,0,0,1,0,0,0,1,1,0,0,1,0,0,0,0,1,0,0,0,0,0,0,0,1,1,1,0,0,0,1,0,0,0,0,0,0,0,0,0$, $0,1,0,0,0,0,0,0,1,0,0,0,0,0,0,0,0,1,0,0,0,1,0,0,0,0,1,0,1,0,1,1,0,1,0,0,0,1,0,0,0,0,0,0,0,0,0,1,0,1,0,1,0,0,1,0$, $1,1,0,1,0,0,0,0,0,0,0,0,0,0,0,0,0,0,0,0,0,1,0,0,1,0,0,0,0,0,0,0,1$,

$0,0,0,0,0,0,0,0,0,0,0,0,0,0,0,0,1,0,0,0,1,1,1,1,1,1,1,1,0,1,1,0,1,1,1,1,1,1,1,1,0,1,1,1,1,1,1,1,1,1,1,1,1,1,1,1$, $1,1,1,1,1,1,1,1,1,1,1,1,1,1,1,1,1,0,1,1,1,1,1,1,1,1,0,0,1,1,1,1,1,0,1,1,1,0,1,1,1,0,1,1,1,1,1,0,1,1,1,1,1,1,1,1$, $1,0,1,1,1,0,1,1,1,1,0,0,1,1,1,1,1,0,1,1,1,1,1,1,1,1,1,1,1,1,1,1,0,0,1,0,0,0,1,1,1,1,1,1,1,0,1,1,1,1,1,0,1,1,1,1$, $1,0,1,1,1,1,1,1,0,0,0,0,1,1,1,1,1,1,1,1,1,1,1,1,1,1,1,1,1,1,1,1,1,0,1,0,1,1,1,1,1,1,1,1,1,0,1,1,1,1,1,1,1,1,1,1$, $1,1,1,1,0,1,1,1,1,1,0,1,1,1,1,1,1,1,1,1,1,1,0,1,1,0,1,1,0,1,1,0,1,1,1,0,1,1,0,1,1,1,0,0,1,0,1,0,0,1,1,0,0,0,0,1$, $0,1,1,1,1,1,1,1,1,1,1,1,1,1,0,1,1,1,0,1,1,1,1,1,1,1,0,1,1,1,0,1,0,0,0,0,0,0,0,0,0,1,0,0,0,0,0,0,1,0,0,0,0,0,0,1$, $0,0,0,0,0,0,1,0,0,0,0,0,0,0,0,0,0,1,1,1,0,1,1,1,0,1,1,1,1,1,1,1,1,1,1,1,1,1,1,1,1,1,1,1,1,1,0,1,1,1,1,1,1,1,0,1$, $0,1,1,1,1,1,0,1,1,1,1,1,1,1,0,1,1,1,1,1,1,0,1,1,1,1,1,1,1,1,1,1,1,1,1,1,1,1,1,1,1,1,1,1,1,1,1,1,0,1,1,1,0,0,1,1$, $1,1,1,1,1,1,1,1,1,1,1,1,1,1,1,1,1,1,1,1,1,1,1,1,0,1,1,1,1,1,1,1,1,0,1,1,1,1,1,1,1,1,1,1,1,1,1,1,1,1,1,1,1,1,1,1$, 
1918

1919

1920

1921

1922

1923

1924

1925

1926

1927

1928

1929

1930

1931

1932

1933

1934

1935

1936

1937

1938

1939

1940

1941

1942

1943

1944

1945

1946

1947

1948

1949

1950

1951

1952

1953

1954

1955

1956

1957

1958

1959

1960

1961

1962

1963

1964

1965

1966
$1,1,1,1,1,1,1,1,1,1,0,1,1,1,1,1,1,1,1,1,1,1,1,1,1,0,1,1,1,1,1,1,0,1,1,1,1,1,1,1,1,0,1,1,1,1,1,0,1,1,1,1,0,1,1,1$, $1,1,1,1,1,1,1,1,1,1,1,1,1,1,1,1,1,1,1,1,1,1,1,1,1,1,1,1,1,1,1,1,1,1,1,1,1,1,1,0,1,1,1,1,1,1,1,1,1,1,1,1,1,1,1,1$, $1,1,1,1,1,1,1,1,1,1,1,1,1,1,1,1,1,1,1,1,1,1,0,1,1,1,1,1,1,1,1,1,1,1,1,1,1,1,1,1,1,1,1,1,1,1,1,1,1,1,1,1,1,1,1,1$, $1,1,1,1,1,1,1,1,1,1,1,1,1,0,1,1,1,1,1,1,1,1,1,1,1,0,0,0,1,1,1,1,1,0,1,0,0,1,0,0,1,1,1,1,1,1,1,0,1,1,1,1,1,0,1,0$, $0,1,1,1,1,1,1,0,1,0,0,1,1,1,1,0,0,1,0,0,0,1,0,0,1,0,1,1,0,1,1,1,1,1,0,0,0,1,0,1,1,1,0,1,1,1,0,1,1,1,1,1,1,1,1,1$, $1,1,1,1,1,1,1,0,1,1,1,1,1,1,1,0,1,1,1,1,1,1,0,1,1,1,1,0,0,1,1,1,1$,

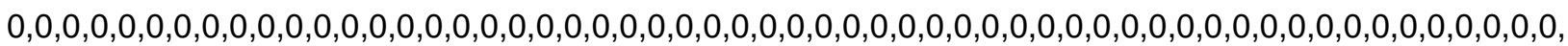
$0,0,0,0,0,0,0,0,0,0,0,0,0,0,0,0,0,0,0,0,0,0,0,0,0,0,0,0,0,0,0,0,0,0,0,0,0,0,0,0,0,0,0,0,0,0,0,0,0,0,0,0,0,0,0,0$, $0,0,0,0,0,0,0,0,0,0,0,0,0,0,0,0,0,0,0,0,0,0,0,0,0,0,0,0,0,0,0,0,0,0,0,0,0,0,0,0,0,0,0,0,0,0,0,0,0,0,0,0,0,0,0,0$, $0,0,0,0,0,0,0,0,0,0,0,0,0,0,0,0,0,0,0,0,0,0,0,0,0,0,0,0,0,0,0,0,0,0,0,0,0,0,0,0,0,0,0,0,0,0,0,0,0,0,0,0,0,0,0,0$, $0,0,0,0,0,0,0,0,0,0,0,0,0,0,0,0,0,0,0,0,0,0,0,0,0,0,0,0,0,0,0,0,0,0,0,0,0,0,0,0,0,0,0,0,0,0,0,0,0,0,0,0,0,0,0,0$, $0,0,0,0,0,0,0,0,0,0,0,0,0,0,0,1,0,0,0,0,0,0,0,0,0,0,0,0,0,0,0,0,0,0,0,0,0,0,0,0,0,0,0,0,0,0,0,0,0,0,0,0,0,0,0,0$, $0,0,0,0,0,0,0,0,0,0,0,0,0,0,0,0,0,0,0,0,0,0,0,0,0,0,0,0,0,0,0,0,0,0,0,0,0,0,0,0,0,0,0,0,0,0,0,0,0,0,0,0,0,0,0,0$, $0,0,0,0,0,0,0,0,0,0,0,0,0,0,0,0,0,0,0,0,0,0,0,0,0,0,0,0,0,0,0,0,0,0,0,0,0,0,0,0,0,0,0,0,0,0,0,0,0,0,0,0,0,0,0,0$, $0,0,0,0,0,0,0,0,0,0,0,0,0,0,0,0,0,0,0,0,0,0,0,0,0,0,0,0,0,0,0,0,0,0,0,0,0,0,0,0,0,0,0,0,0,0,0,0,0,0,0,0,0,0,0,0$, $0,0,0,0,0,0,0,0,0,0,0,0,0,0,0,0,0,0,0,0,0,0,0,0,0,0,0,0,0,0,0,0,0,0,0,0,0,0,0,0,0,0,0,0,0,0,0,0,0,0,0,0,0,0,0,0$, $0,0,0,0,0,0,0,0,0,0,0,0,0,0,0,0,0,0,0,0,0,0,0,0,0,0,0,0,0,0,0,0,0,0,0,0,0,0,0,0,0,0,0,0,0,0,0,0,0,0,0,0,0,0,0,0$, $0,0,0,0,0,0,0,0,0,0,0,0,0,0,0,0,0,0,0,0,0,0,0,0,0,0,0,0,0,0,0,0,0,0,0,0,0,0,0,0,0,0,0,0,0,0,0,0,0,0,0,0,0,0,0,0$, $0,0,0,0,0,0,0,0,0,0,0,0,0,0,0,0,0,0,0,0,0,0,0,0,0,0,0,0,0,0,0,0,0,0,0,0,0,0,0,0,0,0,0,0,0,0,0,0,0,0,0,0,0,0,0,0$, $0,0,0,0,0,0,0,0,0,0,0,0,0,0,0,0,0,0,0,0,0,0,0,0,0,0,0,0,0,0,0,0,0,0,0,0,0,0,0,0,0,0,0,0,0,0,0,0,0,0,0,0,0,0,0,0$,

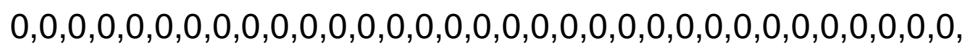

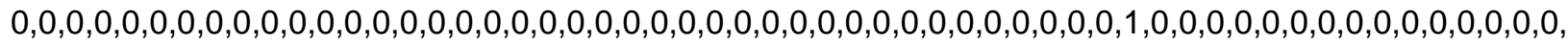
$0,0,0,0,0,0,0,0,0,0,0,0,0,0,0,0,0,0,0,0,0,0,0,0,0,0,0,0,0,0,0,0,0,0,0,0,0,0,0,0,0,0,0,0,0,0,0,1,0,0,0,0,0,0,0,0$, $0,0,0,0,0,1,0,0,0,0,0,0,0,0,0,0,0,0,0,0,0,0,0,0,0,0,0,0,0,0,0,0,0,0,0,0,0,0,0,0,0,0,0,0,0,0,0,0,0,0,0,0,0,0,0,0$, $0,0,0,0,0,0,0,0,0,0,0,0,0,0,0,0,0,0,0,0,0,0,0,0,0,0,0,0,0,0,0,0,0,0,0,0,0,0,0,0,0,0,0,0,0,0,0,0,0,0,0,0,0,0,0,0$, $0,0,0,0,0,0,0,0,0,0,0,0,0,0,0,0,0,0,0,0,0,0,0,0,0,0,0,0,0,0,0,0,0,0,0,0,0,0,0,0,0,0,0,1,0,0,0,0,0,0,0,0,0,0,0,0$, $0,0,0,0,0,0,0,0,0,0,0,0,0,0,0,0,0,0,0,0,0,0,0,0,0,0,0,0,0,0,0,0,0,0,0,0,0,0,0,0,0,0,0,0,0,0,0,0,0,0,0,0,0,0,0,0$, $0,0,0,0,0,0,0,0,0,0,0,0,0,0,0,0,0,0,1,0,0,0,0,0,0,0,0,0,0,0,0,0,0,0,0,0,0,0,0,0,0,0,0,0,0,0,0,0,1,0,0,0,0,0,0,0$, $0,0,0,0,0,0,0,0,0,0,0,0,0,0,0,0,0,0,0,0,0,0,0,0,0,0,0,0,0,0,0,0,0,0,0,0,0,0,0,0,0,0,0,0,0,0,0,1,0,0,0,0,0,0,0,0$, $0,0,0,0,0,0,1,0,0,0,0,0,0,0,0,0,0,0,0,0,0,0,0,0,0,0,0,0,0,0,0,0,0,0,0,0,0,0,1,0,0,0,0,0,0,0,0,0,0,0,0,0,0,0,0,0$, $0,0,0,0,0,0,0,0,0,0,0,0,0,0,0,0,0,0,0,0,0,0,0,0,0,0,0,0,0,0,0,0,0,0,0,0,0,0,0,0,1,1,0,0,0,0,0,0,0,0,0,0,0,0,0,0$, $0,0,0,0,0,0,0,0,0,0,0,0,0,0,0,0,0,0,0,0,0,0,0,0,0,0,0,0,0,0,0,0,0,0,0,0,0,0,0,0,1,0,0,0,0,0,0,0,0,0,0,0,0,0,0,0$, $0,0,0,0,0,0,0,0,0,0,0,0,0,0,0,0,0,0,0,1,0,0,0,0,0,0,0,0,0,0,0,0,0,0,0,0,0,0,0,0,0,0,0,0,0,0,0,0,0,0,0,0,0,0,0,0$, $0,0,0,0,0,1,0,0,0,0,0,0,0,0,0,0,0,0,0,0,0,0,0,0,0,0,0,0,0,0,0,0,0,0,0,0,0,0,0,0,0,0,1,0,0,0,0,0,0,0,0,0,0,0,0,0$, $0,1,0,0,0,0,0,0,0,0,0,0,0,0,0,0,0,0,0,0,0,0,0,0,0,0,0,0,0,0,1,0,0,0,0,0,0,0,0,0,0,0,0,0,0,0,0,0,0,0,0,0,0,0,0,0$, $0,0,0,0,0,0,0,0,0,0,0,0,0,0,0,0,0,1,0,0,0,0,0,0,0,0,0,0,0,0,0,0,0$,

$0,0,0,0,0,0,0,0,0,0,0,0,0,0,0,0,0,0,0,0,0,0,1,1,0,1,0,0,0,1,0,1,0,0,0,1,1,0,0,1,1,0,0,0,0,0,1,0,0,0,0,0,1,0,0,0$, $0,0,0,1,0,0,0,0,0,0,1,0,0,1,0,0,0,1,0,0,0,0,0,0,0,0,0,0,0,0,0,0,0,0,0,0,0,0,0,0,0,1,0,0,0,0,0,0,0,0,1,0,0,1,0,1$, $0,1,0,0,1,0,0,0,0,0,1,0,1,0,1,0,1,1,0,0,0,0,0,0,0,1,0,1,0,0,0,1,1,1,0,1,1,1,0,0,0,0,0,0,1,1,0,0,0,0,0,0,0,1,0,1$, $0,1,0,0,0,0,0,0,1,1,1,0,0,0,1,0,0,0,0,0,1,0,0,0,0,0,0,0,0,0,0,0,1,1,1,1,0,0,1,0,0,0,0,0,0,1,1,0,0,0,0,0,0,0,0,0$, $0,0,0,1,0,0,0,0,1,0,1,0,0,0,0,0,0,0,0,0,0,0,0,1,0,0,0,1,1,1,1,1,0,0,1,1,0,0,0,0,0,0,0,0,0,0,1,0,0,0,0,1,0,1,1,1$, $0,0,0,1,1,1,0,0,0,1,1,1,1,1,0,1,1,0,0,1,0,1,1,0,0,0,0,0,0,0,1,0,1,0,0,0,0,0,0,0,0,0,0,0,0,0,0,0,0,0,0,0,0,0,0,0$, $0,0,0,0,0,0,0,0,0,0,0,0,0,0,0,0,0,1,1,0,0,1,0,0,1,0,0,0,1,0,1,0,0,0,0,0,1,0,0,0,0,0,0,1,0,0,0,0,1,0,0,0,0,0,1,1$, $1,0,0,0,0,0,1,0,0,0,0,0,0,1,0,0,1,0,0,0,0,1,0,0,1,0,0,0,0,1,0,1,0,0,0,0,0,1,0,0,0,0,0,0,0,0,0,1,1,0,0,0,0,0,0,0$, $0,0,0,0,0,0,1,0,0,0,0,0,0,0,0,0,0,0,0,0,0,1,1,0,0,0,0,0,0,0,0,1,0,1,0,0,1,0,1,0,0,0,0,0,0,0,0,0,0,0,0,0,0,0,0,0$, $0,0,0,0,0,0,0,0,0,0,1,0,0,0,0,0,0,1,1,0,0,0,0,0,0,1,1,0,0,0,0,0,1,0,0,0,0,0,0,0,0,0,0,0,0,0,1,1,0,0,0,0,1,0,0,0$, $0,0,0,0,0,0,0,0,0,0,0,0,0,1,0,0,0,0,0,0,0,0,0,0,0,0,0,0,0,1,0,0,0,0,0,0,0,0,0,0,1,0,0,0,1,1,0,1,0,0,0,0,0,1,1,0$, $0,0,1,0,0,0,0,0,1,0,0,0,0,0,1,0,0,0,0,1,1,0,1,0,0,0,0,0,0,0,0,0,0,0,0,0,0,0,0,1,1,0,0,0,0,0,0,0,0,0,0,0,0,0,0,0$, $0,0,0,0,0,1,1,0,0,0,1,0,1,0,1,0,1,0,0,1,1,0,0,1,1,0,0,1,0,0,0,0,1,0,0,0,0,0,0,0,0,1,1,0,0,0,1,0,0,0,0,0,1,0,0,0$, 
1967

1968

1969

1970

1971

1972

1973

1974

1975

1976

1977

1978

1979

1980

1981

1982

1983

1984

1985

1986

1987

1988

1989

1990

1991

1992

1993

1994

1995

1996

1997

1998

1999

2000

2001

2002

2003

2004

2005

2006

2007

2008

2009

2010

2011

2012

2013

2014

2015

$0,1,0,0,0,0,0,0,1,0,0,0,0,0,0,0,0,1,0,0,0,1,0,0,0,0,1,0,1,0,1,1,0,0,0,0,0,1,0,0,0,0,0,0,0,0,0,1,1,1,0,1,0,0,1,0$, $1,1,0,1,1,1,1,0,0,0,1,0,0,0,0,0,0,0,0,0,0,0,0,1,1,0,0,0,0,1,0,0,1$,

$0,0,0,0,1,0,0,0,1,0,0,0,0,0,0,0,0,0,0,0,1,1,0,0,1,1,1,0,0,1,1,0,1,1,0,1,0,1,1,0,0,1,1,1,0,1,1,1,1,1,1,1,0,1,1,1$, $1,1,0,1,1,0,1,1,1,1,1,0,1,0,1,1,1,0,1,1,1,1,1,1,1,1,0,0,1,1,1,1,1,1,1,1,1,1,1,1,1,0,1,1,1,1,1,0,1,1,0,1,1,0,1,0$, $1,1,1,1,0,1,1,1,1,1,0,0,1,1,0,1,1,0,1,1,1,1,1,1,1,0,1,1,1,1,1,1,1,1,1,1,1,1,1,1,1,1,1,1,1,1,0,1,1,1,1,0,1,0,1,1$, $1,0,1,1,1,1,1,1,0,0,0,0,1,1,1,1,1,1,1,1,0,1,1,1,1,1,1,1,1,1,1,1,0,1,1,0,1,1,0,1,1,1,1,1,1,1,0,1,1,0,1,1,1,1,1,1$, $1,1,1,0,0,1,1,1,0,1,0,1,1,1,1,1,1,1,1,1,1,1,0,0,1,0,0,0,0,1,1,0,1,1,1,1,1,1,0,1,1,1,0,1,1,0,1,0,0,1,1,1,0,1,1,1$, $0,0,1,1,1,1,1,1,1,1,1,1,1,1,0,0,1,0,1,1,1,0,1,1,1,1,1,0,1,1,0,1,0,0,0,0,0,0,0,0,0,0,0,0,0,0,0,0,0,0,0,0,0,0,0,0$, $0,0,0,0,0,1,0,0,0,0,0,0,0,0,0,0,0,1,0,1,0,0,1,1,1,1,1,1,0,1,0,1,1,1,1,1,0,1,1,1,1,1,1,0,1,1,0,1,0,1,1,1,1,1,0,0$, $0,1,0,1,1,1,0,1,1,1,0,1,1,0,0,1,0,1,1,1,1,0,1,1,0,1,0,1,1,0,1,0,1,1,1,1,1,0,1,1,1,1,1,1,1,1,1,0,0,1,1,1,0,0,1,1$, $1,1,1,1,1,0,0,1,1,1,1,1,1,1,1,1,1,1,1,1,1,0,1,1,0,1,1,1,1,1,1,0,1,1,1,1,0,1,0,1,1,1,1,1,1,1,1,1,1,1,1,1,1,1,1,1$, $1,1,1,1,1,1,1,1,1,1,0,1,1,1,1,1,1,0,0,1,1,1,1,1,1,1,0,1,1,1,1,1,0,1,1,1,1,1,1,1,0,0,1,1,1,1,0,0,1,1,1,1,0,1,1,1$, $1,1,1,1,1,1,1,1,1,1,1,1,1,1,1,1,1,1,1,1,1,1,1,1,1,1,1,1,1,0,1,1,1,1,1,1,1,1,0,0,0,1,1,1,0,0,1,0,1,1,1,1,1,0,0,1$, $1,1,1,1,1,1,1,1,1,1,1,1,1,1,1,1,1,1,1,0,0,1,0,1,1,0,1,1,1,1,1,1,1,1,1,1,1,1,1,0,0,1,1,1,1,1,1,1,1,0,1,1,1,1,1,1$, $1,1,1,1,1,0,0,1,1,1,0,1,0,0,1,1,1,1,1,0,1,1,0,0,1,0,0,0,1,1,1,1,0,0,1,0,0,1,0,0,1,1,1,1,1,1,1,0,1,1,1,1,0,0,1,0$, $0,1,1,1,1,1,1,0,1,0,0,1,1,1,1,0,0,1,0,0,0,0,0,0,1,0,0,1,0,1,1,1,1,0,0,0,0,1,0,1,1,1,0,1,1,1,0,1,0,1,1,1,1,1,1,1$, $1,1,1,1,1,1,1,0,1,1,1,1,1,1,1,0,1,1,1,1,1,1,0,1,1,1,1,0,0,1,1,1,0$,

$0,0,0,0,0,0,0,0,0,0,0,0,0,0,0,0,0,0,0,0,1,1,1,1,1,1,1,1,1,0,1,1,1,1,1,1,1,1,1,1,1,1,1,1,1,1,1,1,1,1,1,1,1,1,1,1$, $1,1,1,1,1,1,1,1,1,1,1,1,1,1,1,1,1,0,1,1,1,1,1,1,1,1,1,0,1,1,1,1,1,1,1,1,1,1,1,1,1,0,1,1,1,1,1,1,1,1,1,1,1,1,1,1$, $1,1,1,1,0,1,1,1,1,1,1,1,1,1,1,1,1,0,1,1,1,1,1,1,1,1,1,1,1,1,1,1,1,1,1,1,1,1,1,1,1,1,1,1,1,0,1,1,1,1,1,1,1,0,1,1$, $1,0,1,1,1,1,1,1,0,0,1,1,1,1,1,1,1,1,1,1,1,1,1,1,1,1,1,1,1,1,1,1,1,1,1,0,1,1,1,1,1,1,1,1,1,1,1,1,1,1,1,1,1,1,1,1$, $1,1,1,1,1,1,1,1,1,1,1,1,1,1,1,1,1,1,1,1,1,1,1,1,1,0,1,1,1,1,1,1,1,1,1,1,1,1,0,1,1,1,0,1,1,0,1,0,0,1,1,1,0,1,0,1$, $0,1,1,1,1,1,1,1,1,1,1,1,1,1,0,1,1,1,1,1,1,1,1,1,1,1,0,1,1,1,1,1,0,0,0,0,0,0,0,0,0,0,0,0,0,0,0,0,0,0,0,0,0,0,0,0$, $0,0,0,0,0,0,0,0,0,0,0,0,0,0,0,0,0,1,1,1,1,1,1,1,0,1,1,1,1,1,1,1,1,1,1,1,1,1,1,1,1,1,1,1,1,1,0,1,1,1,1,1,1,1,1,1$, $1,1,1,1,1,1,1,1,1,1,1,1,1,1,1,1,1,1,1,1,1,0,1,1,1,1,1,1,1,1,1,1,1,1,1,1,1,0,1,1,1,1,1,1,1,1,1,1,1,1,1,1,1,1,1,1$, $1,1,1,1,1,1,1,1,1,1,1,1,1,1,1,1,1,1,1,1,1,1,1,1,1,1,1,1,1,1,1,1,1,1,1,1,1,1,1,1,1,1,1,1,1,1,1,1,1,1,1,1,1,1,1,1$, $1,1,1,1,1,1,1,1,1,1,1,1,1,1,1,1,1,1,1,1,1,1,1,1,1,0,1,1,1,1,1,1,1,1,1,1,1,1,1,1,1,1,1,1,1,1,1,0,1,1,1,1,1,1,1,1$, $1,1,1,1,1,1,1,1,1,1,1,1,1,1,1,1,1,1,1,1,1,1,1,1,1,1,1,1,1,1,1,1,1,1,1,1,1,1,1,1,1,1,1,1,1,1,1,1,1,1,1,1,1,1,1,1$, $1,1,1,1,1,1,1,1,1,1,1,1,1,1,1,1,1,1,1,1,1,1,1,1,1,1,1,1,1,1,1,1,1,1,1,1,1,1,1,1,1,1,1,1,1,1,1,1,1,1,1,1,1,1,1,1$, $1,1,1,1,1,1,1,1,1,1,1,1,1,0,1,1,1,1,1,1,1,1,1,1,1,0,0,1,1,1,1,1,1,0,1,0,0,1,0,0,1,1,1,1,1,1,1,0,1,1,1,1,1,0,1,0$, $0,1,1,1,1,1,1,0,1,0,0,1,1,1,1,0,0,1,0,0,0,1,0,0,1,0,1,1,0,1,1,1,1,1,0,0,0,1,0,1,1,1,0,1,1,1,0,1,1,1,1,1,1,1,1,1$, $1,1,1,1,1,1,1,0,1,1,1,1,1,1,1,0,1,1,1,1,1,1,0,1,1,1,1,0,0,1,1,1,1$,

0, $0,0,0,0,0,0,0,0,0,0,0,0,0,0,0,0,0,0,0,0,0,0,0,0,0,0,0,1,0,0,0,0,0,0,0,0,0,0,0,1,0,0,0,0,0,0,0,0,0,0,0,0,0,0,0$, $1,0,0,0,0,0,0,0,0,0,0,0,0,0,0,0,0,0,0,0,0,0,0,0,0,0,0,0,0,0,0,0,0,0,0,0,0,0,0,0,0,0,0,0,0,0,0,0,0,0,0,0,0,0,0,0$, $0,0,0,0,0,0,0,0,0,0,0,0,0,0,0,0,0,0,0,0,0,0,0,0,0,0,0,0,0,0,0,0,0,0,0,0,0,0,0,0,0,0,0,0,0,0,0,0,0,0,0,0,0,0,0,0$, $0,0,0,0,0,0,0,1,0,1,0,0,1,0,0,0,0,0,1,0,0,0,0,0,0,0,0,0,0,0,0,0,0,0,0,0,0,0,0,0,0,0,0,0,0,0,0,0,0,0,0,0,0,0,0,0$, $0,0,0,0,0,0,0,0,0,0,0,0,0,0,0,0,0,0,0,0,0,0,0,0,0,0,0,0,0,0,0,0,0,0,0,0,0,0,0,0,0,0,0,0,0,0,0,0,0,0,0,0,0,0,0,0$, $0,0,0,0,0,0,0,0,0,0,1,1,0,0,0,0,0,0,0,0,0,0,0,0,0,0,0,0,0,0,0,0,0,0,0,0,0,0,0,0,0,0,0,0,0,0,0,0,0,0,1,0,0,0,0,0$, $0,0,0,0,0,0,0,0,0,0,0,0,0,0,0,0,0,0,0,0,0,0,0,0,0,0,0,0,0,0,0,0,0,0,0,0,0,0,0,0,0,0,0,0,0,0,0,0,0,0,0,0,0,0,0,0$, $0,0,0,0,0,0,0,0,0,0,0,0,0,0,0,0,0,0,0,0,0,0,0,0,0,0,0,0,0,0,0,0,1,0,1,1,0,0,0,0,0,0,0,0,1,0,0,0,0,0,0,0,0,0,0,0$, $0,0,0,0,0,0,0,0,0,0,0,0,0,0,0,0,0,0,0,0,0,0,0,0,0,0,0,0,0,0,0,0,0,0,0,0,0,0,0,0,0,0,0,0,0,0,0,0,0,0,0,0,0,0,0,0$, $0,0,0,0,0,0,0,0,0,0,0,0,0,0,0,0,0,0,0,0,0,0,0,0,0,0,0,0,0,0,0,0,0,0,0,0,0,0,0,0,0,0,0,0,0,0,0,0,0,0,0,0,1,0,0,0$, $0,0,0,0,0,0,0,0,0,0,0,0,0,0,0,0,0,0,0,0,0,0,0,0,0,0,0,0,0,0,0,0,0,0,0,0,0,0,0,0,0,0,0,0,0,0,0,0,0,0,0,0,0,0,0,0$, $0,0,0,0,0,0,0,0,0,0,0,0,0,0,0,0,0,0,0,0,0,0,0,0,0,0,0,0,0,0,0,0,0,0,0,0,0,0,0,0,0,0,0,0,0,0,0,0,0,0,0,0,0,0,0,0$, $0,0,0,0,0,0,0,0,0,0,0,0,0,0,0,0,0,0,0,0,0,0,0,0,0,0,0,0,0,0,0,0,0,0,0,0,0,1,0,0,0,0,0,0,1,0,0,0,0,0,0,0,0,0,0,0$, $0,0,0,1,0,0,0,0,0,0,0,0,0,0,0,0,0,0,0,0,0,0,0,0,0,0,0,0,0,0,0,0,0,0,0,0,0,0,0,0,0,0,0,0,0,0,0,0,0,0,0,0,0,0,0,0$, $0,0,0,0,0,0,0,0,0,0,0,0,0,0,0,0,0,0,0,0,0,0,0,0,0,0,0,0,0,0,0,0,0$

). $. \operatorname{Dim}=c(12,817)))$ 
2016

2017

2018

2019

2020

2021

$2022)$,. $\operatorname{Dim}=c(12, X X)))$

2023

2024 \# INITIAL VALUES

2025

2026 list $($ PriorS $=c(0.1,0.1,0.1,0.1,0.1,0.1,0.1,0.1,0.1,0.1,0.1,0.1,0.1,0.1,0.1,0.1,0.1,0.1,0.1))$

2027

2028 list(PriorS

$2029=c(0.01,0.01,0.01,0.01,0.01,0.01,0.01,0.01,0.01,0.01,0.01,0.01,0.01,0.01,0.01,0.01,0.01,0.01,0.01))$

2030

2031 list(PriorS $=c(0.2,0.2,0.2,0.2,0.2,0.2,0.2,0.2,0.2,0.2,0.2,0.2,0.2,0.2,0.2,0.2,0.2,0.2,0.2))$

2032

2033 list(PriorS

$2034=\mathrm{c}(0.05,0.05,0.05,0.05,0.05,0.05,0.05,0.05,0.05,0.05,0.05,0.05,0.05,0.05,0.05,0.05,0.05,0.05,0.05))$

2035

2036 list $($ PriorS $=c(0.3,0.3,0.3,0.3,0.3,0.3,0.3,0.3,0.3,0.3,0.3,0.3,0.3,0.3,0.3,0.3,0.3,0.3,0.3))$ 